A N N A L E S Annales de Bretagne et des Pays de l'Ouest

Anjou. Maine. Poitou-Charente. Touraine

118-3 | 2011

La naissance de l'archéologie régionale dans l'Ouest armoricain

\title{
Fortuné Parenteau (1814-1882) et Pitre de Lisle du Dreneuc (1846-1924) collectionneurs et conservateurs passionnés
}

Fortuné Parenteau (1814-1882) and Pistre de Lisle du Dreneuc (1846-1924), passionnate collectors and first curators

Marie-Hélène Santrot

\section{(2) OpenEdition}

Journals

Édition électronique

URL : http://journals.openedition.org/abpo/2065

DOI : $10.4000 / a b p o .2065$

ISSN : 2108-6443

Éditeur

Presses universitaires de Rennes

Édition imprimée

Date de publication : 30 septembre 2011

Pagination : 151-242

ISBN : 978-2-7535-1770-7

ISSN : 0399-0826

Référence électronique

Marie-Hélène Santrot, «Fortuné Parenteau (1814-1882) et Pitre de Lisle du Dreneuc (1846-1924) collectionneurs et conservateurs passionnés ", Annales de Bretagne et des Pays de l'Ouest [En ligne], 118-3 | 2011, mis en ligne le 30 novembre 2013, consulté le 21 avril 2019. URL : http:// journals.openedition.org/abpo/2065; DOI : 10.4000/abpo.2065 


\title{
Fortuné Parenteau (1814-1882) et Pitre de Lisle du Dreneuc (1846-1924) collectionneurs et conservateurs passionnés
}

\author{
Marie-Hélène SANTROT \\ Musée Dobrée, Nantes
}

"Collectionner, c'est vivre" Fortuné Parenteau, $1858^{1}$

\begin{abstract}
Deux personnalités attachantes et fort différentes, Fortuné Parenteau du Payré (Luçon, 7 avril 1814 - Pouzauges, 10 septembre 1882) et Pitre (Pierre) de Lisle du Dreneuc (Nantes, 24 avril 1846- Nantes, 12 février 1824) ont marqué l'archéologie locale et le musée départemental d'Archéologie, à Nantes, aujourd'hui le musée Dobrée, dans la seconde moitié du XIX ${ }^{\mathrm{e}}$ siècle et le premier quart du XIX ${ }^{e}$. Dans le cadre des sociétés savantes qui se développent à partir du milieu du XIX ${ }^{\mathrm{e}}$ siècle, et tout particulièrement des sociétés archéologiques, les érudits locaux trouvent l'occasion de glaner les traces des civilisations anciennes grâce, notamment, aux travaux d'aménagement urbain en cours. L'émulation qui anime les membres de ces sociétés est la formation de collections privées nombreuses et riches, dans la tradition lointaine de la Renaissance italienne et des "cabinets de curiosité". En dépit d'erreurs dues aux méthodes de fouilles pratiquées alors, qui ont souvent entraîné une destruction des archives du sol, ces "pionniers" de l'archéologie ont répertorié les sites rencontrés et préservé leurs mobiliers. Ainsi, par leur goût de la collection, ces inventeurs, comme Pitre de Lisle du Dreneuc, ont réuni le mobilier exhumé lors des fouilles ou à l'occasion de découvertes fortuites. Fortuné Parenteau, quant à lui, a également beaucoup enrichi sa collection par achat ou par échange, comme cela se pratiquait fréquemment entre collectionneurs. Conservateurs et collectionneurs, chacun à
\end{abstract}

1. Parenteau, Fortuné, Catalogue descriptif d'une collection de bijoux, 1858, musée Dobrée, fonds Parenteau, 1997, inv. 997.11.9, p. 268. 
sa manière, Fortuné Parenteau (fig. 1) et Pitre de Lisle du Dreneuc (fig. 2) se sont attachés à mener de front leurs deux passions - qui se sont parfois révélées antinomiques - mais qui ne font qu'une à leurs yeux : l'archéologie, pour la connaissance et pour le musée, et leur collection personnelle.

Figure 1 - Fortuné Parenteau, laissez-passer à l'Exposition universelle de Paris, 1878 (musée Dobrée, inv. 58.554 - cl. musée Dobrée)

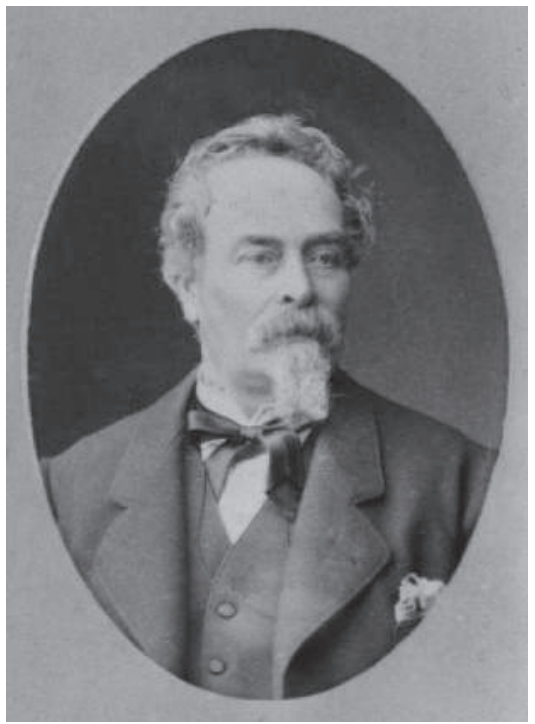

Figure 2 - Pitre de Lisle du Dreneuc, laissez-passer à l'Exposition universelle de Paris, 1878 (coll. privée - cl. musée Dobrée)

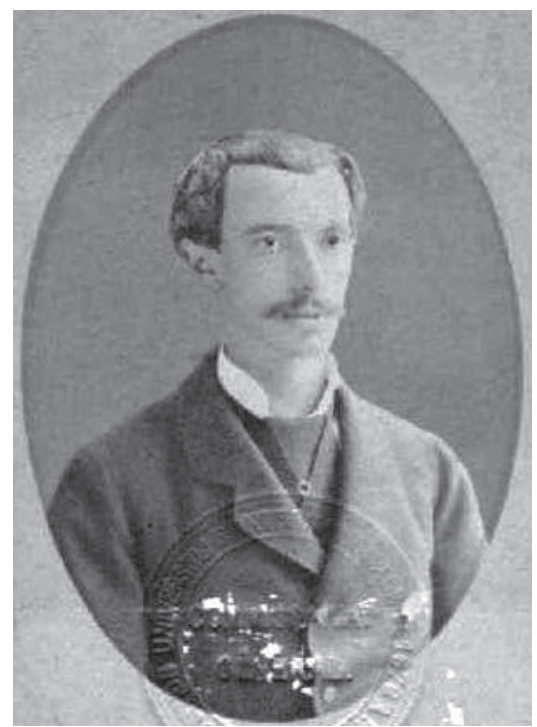

Si l'on ne sait pas grand-chose de leur vie, en revanche, d'importantes archives, récemment acquises par le musée Dobrée, fournissent un éclairage intéressant sur Fortuné Parenteau, ses "fouilles", ses méthodes d'acquisition et la constitution de sa collection personnelle dont il fit don au musée archéologique en 1881. La découverte récente, en mains privées, d'un carnet de journal personnel illustré de Pitre de Lisle du Dreneuc pour 1877-18872 lève un pan du voile sur ses méthodes de collecte et son goût très vif de la "chasse" aux objets archéologiques, tandis que sa collection personnelle et celle de son frère Georges, vendues au marquis Pierre Auguste de Montaigu en 1903, nous sont connues par le catalogue édité à cette occasion et par l'important don du reliquat de cette collection et d'ar-

2. Nous remercions très chaleureusement $\mathrm{M}^{\text {me }}$ Alice Debenay, arrière-petite-fille de Pitre, de nous avoir donné accès aux archives et souvenirs de famille de l'archéologue, conservés par son père, le colonel de la Fouchardière, aujourd'hui décédé. 
chives au musée Dobrée en 1993 par Paulette Dommée ${ }^{3}$. Plus encore que pour Fortuné Parenteau, cette documentation permet de mieux appréhender sa difficulté à établir une limite entre les collections du musée départemental d'Archéologie et sa collection privée.

Quoi qu'il en soit, tous deux vont jeter les bases d'un musée archéologique départemental à Nantes, précédemment musée de la Société archéologique de Nantes et de la Loire-Inférieure de 1849 à 1860. Installé d'abord rue du Moulin par la Ville, puis à la chapelle de l'Oratoire, ce musée associatif est devenu départemental en 1860 par le don au Département des collections de la Société archéologique, puis a été réuni au musée Dobrée et transféré en 1897 dans les bâtiments légués par l'ancien armateur Thomas Dobrée.

\section{Fortuné Parenteau (1814-1882)}

\section{Les années de formation, 1830-1859}

Avant 1851 et son arrivée à Nantes, Fortuné Parenteau est, selon les textes ${ }^{4}$, considéré comme " avocat ", " propriétaire " ou " rentier ${ }^{5}$ ". L'étude de ses " carnets " nous montre qu'il fait bien exploiter des propriétés agricoles en Vendée, les siennes et celles de son beau-père, car, tous les mois (et sur les mêmes pages!), il reporte à la fois ses comptes de fermages et ceux du musée. En 1830, à la fin de ses études (il a 16 ans), il part à Paris où il séjourne une dizaine d'année pour assister aux cours du Collège de France ${ }^{6}$. À son retour, il s’installe à Luçon (Vendée), ainsi que l'écrit Pitre de Lisle du Dreneuc dans sa biographie : "Il était donc admirablement préparé pour les recherches qu'il allait entreprendre ${ }^{7}$. " Formé localement par deux érudits vendéens ${ }^{8}$, le spécialiste des monnaies féodales Faustin Poëy d'Avant et le juriste, historien de l'art, archéologue et collectionneur Benjamin Fillon, neveu par alliance de Poëy d'Avant, Fortuné Parenteau

3. Son mari, Claude Dommée, avait acquis en 1964 les restes de la collection archéologique du musée privé du château de la Bretesche en Missillac (Loire-Atlantique) précédemment dispersée par le marquis Philippe de Montaigu : BLAIN, Hugues-François et SANTROT, Jacques, " Gustave Paille, un archéologue "à façon" en Basse Bretagne (18981905) ", dans $A B P O, 107,2000,3$, p. 102, n. 1-3.

4. Dousset, Léonce, Généalogie de la famille Parenteau, Poitiers, Soc. Française d'imprimerie et de librairie, 1913 (avocat) ; " Annuaire " de la Société d'Émulation de la Vendée, entre 1871 et 1883, où il est qualifié de "propriétaire " ou de "rentier ".

5. Né le 7 avril 1814 à Luçon, il est le fils de Pierre Parenteau Dupayré (à son mariage et à son décès inscrit " $d u$ Payré "), propriétaire, et de Marie Thérèse Saboureau (ou Sabouraud) Desaubière. Il a un frère jumeau, Auguste, qui est son aîné : Archives départementales de la Vendée, état-civil de Luçon, AD2E128/11, années 1811-1814.

6. Dans "Cabournes, bourniers et bournigals ", BSANLI, 12, 1873, p. 29, il écrit : "De 1830 à 1840, je suivais comme étudiant les cours du collège de France et j'ai toujours conservé souvenance d'une leçon de M. Michelet. Il était question d'histoire et de migrations des peuples..."

7. Lisle du Dreneuc, Pitre de, Revue de Bretagne et de Vendée, LII, 1882, p. 471.

8. Musée Dobrée, fonds Parenteau, 1997, inv. 997.11.5, p. 1. 
commence une collection de bijoux, puis de monnaies qu'il poursuit pendant une dizaine d'années ${ }^{9}$.

En 1851, après son mariage avec Louise Augustine Sidonie Brunet ${ }^{10}$, Parenteau quitte sa Vendée natale pour s'installer à Nantes. Depuis quelques temps déjà il avait rejoint le grand mouvement naissant des Sociétés savantes locales : la Société des Antiquaires de l'Ouest (créée en 1834 et qu'il rejoint en 1850), la Société d'Émulation de Vendée (créée en 1854 et où il entre en 1855), et enfin, la Société archéologique de Nantes et de la Loire-Inférieure, fondée en 1845 émanation d'abord de l'Association bretonne (section nantaise d'archéologie), puis association archéologique, à laquelle il est admis le 3 septembre $1851^{11}$. Son nom paraît dans les comptes-rendus des séances de la Société archéologique de Nantes et de la Loire-Inférieure dès l'année suivante où il fait la présentation d'un rapport sur les médailles conservées à l'hôtel de ville, et devient donateur ${ }^{12}$. Son nom paraît ensuite très régulièrement dans les comptes-rendus de séances jusqu'en 1856 (dons divers d'objets archéologiques). Enfin, à la séance du 6 décembre 1854, Parenteau devient membre du bureau de la Société archéologique avec Armand Guéraud, Émile Pradal, Louis Séraphin Le Ray, Arthur Le Moyne de La Borderie et Charles Marie Armand d'Izarn. À ce titre, lors de la séance du 24 avril 1855, il prend de l'assurance et participe à un débat majeur sur le musée de la Société :

" M. Nau [Théodore-Jacques Nau, architecte diocésain, président de la Société archéologique de Nantes depuis sa création] entretient le comité [membres du bureau de la Société archéologique] d'un projet d'établissement du musée archéologique dans la chapelle de l'Oratoire. Il développe les raisons qui lui semblent devoir rendre désirable l'appropriation de ce monument, désormais inutile au culte, au musée archéologique de Nantes. Il soumet au comité les deux questions. Le musée devrait-il être départemental ou communal? La chapelle de l'Oratoire convient-elle à l'établissement d'un musée archéologique?

M. Parenteau insiste sur les avantages de la position. Il pense que le musée a tout intérêt à être proclamé départemental. Cette qualité avait pour résultat nécessaire de rattacher la convention du musée aux intérêts généraux du Département. M. Parenteau désirerait connaître si le monument proposé conviendrait aux séances de la Société. M. le Président croit qu'à ce point de

9. Musée Dobrée, fonds Parenteau, 1997, inv. 997.11.5, p. 2. Parenteau nous y apprend que le premier bijou de sa collection est une petite boucle d'oreille en or découverte avec un trésor monétaire mérovingien au château de Beaugisière, près de Fontenay-le-Comte (Vendée) ; il y fait placer au centre une intaille antique en pâte de verre.

10. À l'âge de 37 ans, Parenteau épouse Louise Augustine Sidonie Brunet à Pouzauges le 8 juin 1851. Fille du maire de la commune, elle est âgée de 21 ans. Dans l'acte civil, Fortuné Parenteau est désigné par son beau-père comme " propriétaire ": Archives départementales de la Vendée, état-civil de Pouzauges, AD2E182/8, années 1849-1854.

11. Bulletin de la Société archéologique de Nantes et de la Loire-Inférieure (BSANLI), 1859, I, p. 5 ; BSANLI, II, 1862, p. 10, 3 septembre 1851 ; on y apprend qu'il y est admis en même temps que MM. de Sesmaisons et de Saint-Pern.

12. BSANLI, II, 1862, p. 12, 2 mars et 6 avril 1852. Il offre un vase à la Société (sans précision). 
vue ce local offre toute convenance dans la partie de la basse nef. M. Vandier rappelle que, lors de l'établissement du musée archéologique, les vœux s'étaient portés sur la chapelle de l'Oratoire. On fut à cette époque effrayé des dépenses qu'aurait entraînées la restauration du monument dont il s'agit ${ }^{13}$. "

Parallèlement Fortuné Parenteau se fait connaître par ses premières publications : en 1856, dans la Revue de l'Ouest, il s'intéresse aux monnaies baronnales dans un article intitulé la "Découverte des Échaubroignes " (aujourd'hui, Les Échaubrognes), qu'il illustre de planches dessinées de sa main. Ces planches firent l'admiration (avec une pointe d'envie) de Pitre de Lisle du Dreneuc qui en fait l'éloge :

"Les gravures de F. Parenteau ont, au contraire, une étrange variété, elles ne donnent pas seulement l'effigie des médailles, elles reproduisent l'image fidèle de la pièce elle-même, indiquant le relief des caractères, ou les parties effacées et mal venues à la frappe ${ }^{14}$."

Cette aptitude au dessin lui permet également d'illustrer de 26 planches de monnaies la publication de son ami Poëy d'Avant sur les monnaies seigneuriales de France, parue en 1853, et ainsi de garder d'excellentes relations avec la Vendée ${ }^{15}$. C'est également en Vendée qu'il pratique ses premières "fouilles" archéologiques : à Vairé, en 1855, puis à Pouzauges en 1858. Il fouille également à Rezé en 1857-1858, où, même s'il ne tient pas de carnets de fouilles, il dessine les structures et les objets découverts : bas-relief de Rezé ${ }^{16}$ (fig. 3), brique mérovingienne de la collection de Benjamin Fillon ${ }^{17}$ (fig. 4), Vénus anadyomène trouvée à Nantes ${ }^{18}$ (fig. 5), etc.

Entre 1845 et 1854, ses carnets de notes sont remplis de dessins d'objets, pas seulement de bijoux et de monnaies, provenant de sites français ou étrangers, bien évidemment de Vendée, mais aussi de Rennes, de Nîmes, ou encore de la villa gallo-romaine de Hartlip (Comté de Kent, Angleterre). Il se «fait la main et l'œil » aux objets archéologiques. Sa méthode de documentation consiste à dessiner tout ce qui l'intéresse et à découper et coller des articles sur tout ce qui se rapporte à ses recherches.

En revanche, Parenteau n'est pas encore admis à participer au grand œuvre des membres de la Société archéologique de Nantes et de la

13. BSANLI, III, 1863, p. 14, 6 décembre 1854.

14. LISLE Du DRENEUC, Pitre de, RBV, LII, 1882, p. 472.

15. Faustin Poëy d'Avant, receveur de l'enregistrement des Domaines à Luçon, est témoin à son mariage le 8 juin 1851 : Archives départementales de la Vendée, état-civil de Pouzauges, AD2E182/8, années 1849-1854.

16. Musée Dobrée, fonds Parenteau, 1997, inv. 997.11.29, p. 11. Parenteau interprète mal ce bas-relief sur lequel il voit une tête grossière, qu'il pense être de l'époque mérovingienne, et prend les phallus ailés pour des bras avec de grandes mains.

17. Musée Dobrée, fonds Parenteau, 1997, inv. 997.11.32, p. 55, "Brique franke trouvée à Rezai, 1849 Lire inf. - communiquée par M. Fillon. "Cette pièce n'est pas entrée dans les collections du musée.

18. Musée Dobrée, fonds Parenteau, 1997, inv. 997.11.29, p. 15; cette pièce n'est pas entrée dans les collections du musée. 
Figure 3 - Bas relief au buste et aux phallus ailés de Rezé (L.-A.)

(Fortuné Parenteau, 1849, musée Dobrée, fonds Parenteau, inv. 997.11.29, $n^{\circ} 2$, p. 11 - cl. musée Dobrée)

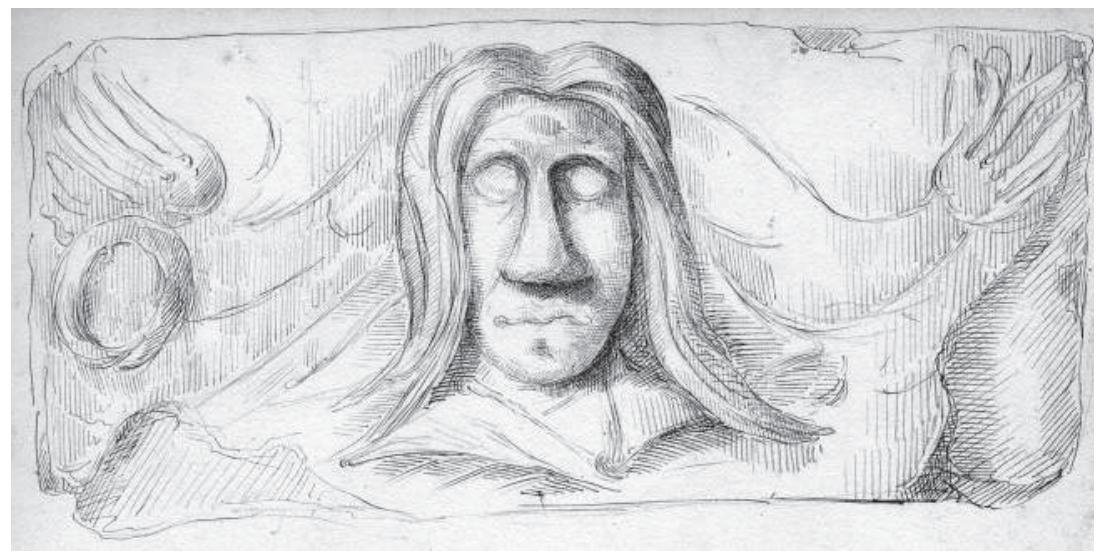

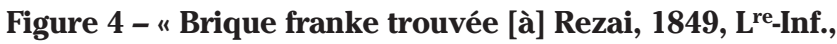
communiquée par M· Fillon "

(Fortuné Parenteau, 1849, musée Dobrée, fonds Parenteau, inv. 997.11.32, $n^{\circ} 5$, p. 55 - cl. musée Dobrée)

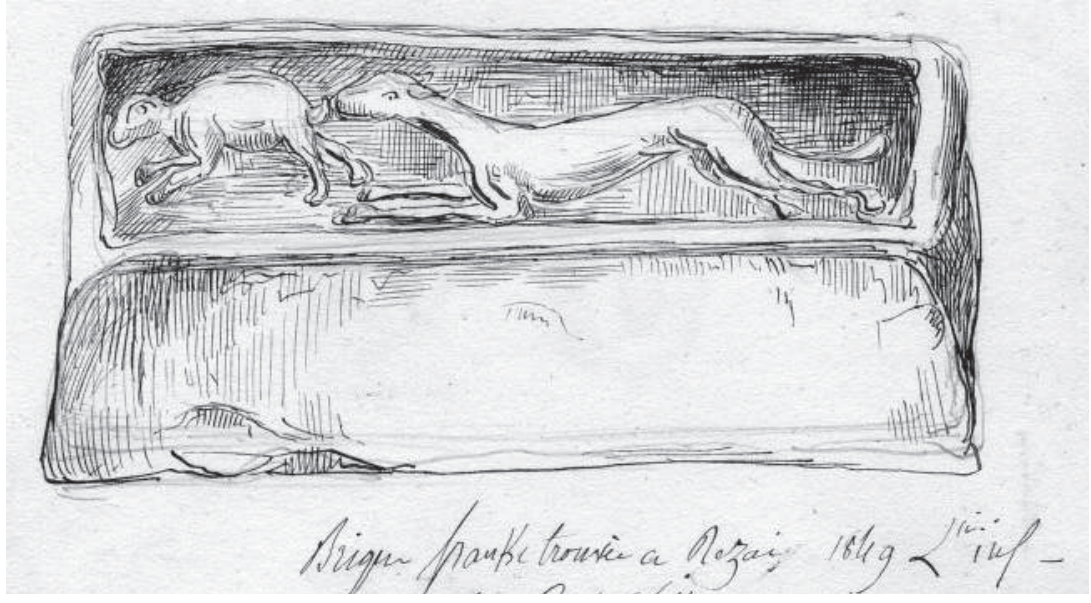

Loire-Inférieure : poursuivre l'étude et la conservation des antiquités de Nantes et de ses environs, rassemblées ou consignées, depuis la fin du $\mathrm{XVIII}^{\mathrm{e}}$ siècle, par les architectes voyers de la Ville, et notamment PierreNicolas Fournier, à l'occasion du percement de voies ou de l'implantation d'égouts. Les sociétaires s'attachent à recueillir les traces du passé à l'occasion des grands travaux urbains et contribuent aussi à écrire l'his- 
Figure 5 - " Terre cuite, Mauves (Nantes, 1874), Vénus anadyomène, Mauves (L.-A.)" (Fortuné Parenteau, 1874, musée Dobrée, fonds Parenteau, inv. 997.11.29, $n^{\circ} 2, p .15$ - cl. musée Dobrée)

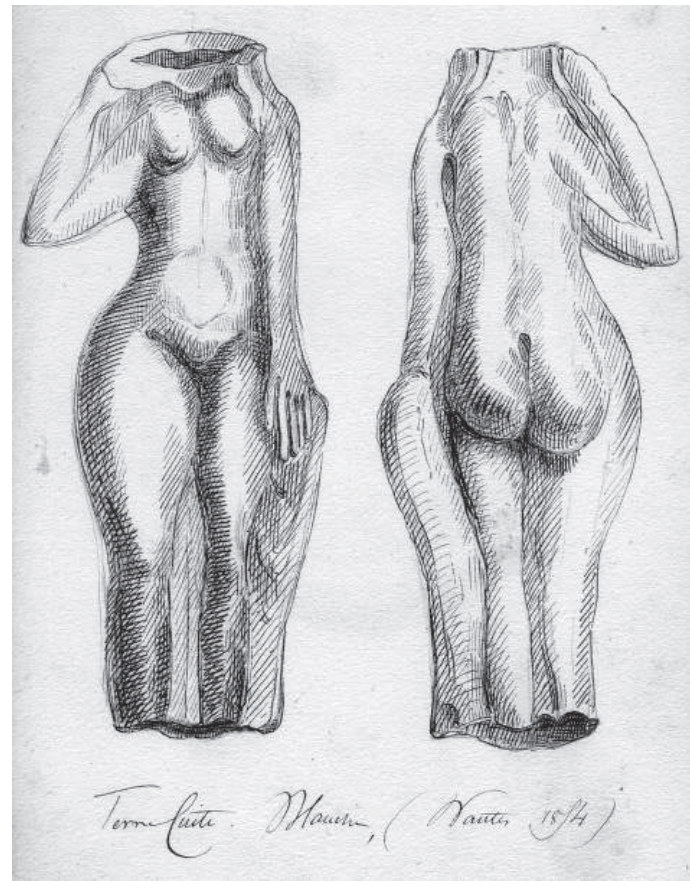

toire locale en dépouillant les archives. Ils surveillent les terrassements, mènent quelques fouilles et, dès 1845 , sauvent ce qu'ils peuvent des vestiges archéologiques, tandis que, depuis 1846, dans un souci pédagogique, l'abbé Henri Rousteau dispense, pour la Société, des cours gratuits d'archéologie.

Dès $1849^{19}$, une salle municipale, la salle de dessin de la maison communale du 18 de la rue du Moulin ${ }^{20}$, héberge les objets recueillis par la Société et huit de ses membres sont désignés pour classer les objets accumulés depuis 1845. Inauguré le $1^{\mathrm{er}}$ mai 1849, ce " dépôt pour la formation du Musée futur $^{21}$ " n'est alors accessible qu'aux seuls spécialistes ${ }^{22}$. L'archiviste départemental Jules Théodore Vandier est nommé le 3 mai 1850 conservateur de

19. Le 3 septembre 1846, la Société archéologique avait déjà demandée au Conseil général de Loire-Inférieure la mise à disposition du local, municipal, de la chapelle de l'Oratoire pour y entreposer ses collections archéologiques; ce qui lui avait été refusé : BSANLI, 1, 1859, p. 143, 3 septembre 1846.

20. "Musée départemental d'Archéologie ", dans La Ville de Nantes et la Loire-Inférieure, II, Nantes, 1898, p. 71-81.

21. BSANLI, I, 1859, p. 256, 5 janvier 1849 : le président de la Société archéologique, Théodore Nau, observe qu'il ne peut pas encore avancer le mot de musée.

22. VANDIER, Jules Théodore, " Notice sur la Société archéologique de Nantes ", BSANLI, 1859 , I, p. 29-30. 
ces collections. Il inscrit à l'inventaire (Registre énonciatif ${ }^{23}$ ) les 648 premiers numéros, soit 1176 objets, tandis que le médaillier compte déjà 958 médailles et monnaies ${ }^{24}$. Il précise que l'espace est déjà trop petit :

"L'espace a manqué pour classer les objets méthodiquement, et cette application de la méthode est forcément ajournée jusqu'à la possession d'un local plus étendu, et convenablement approprié à sa destination " et encore " ce local insuffisant n'a pas permis d'y étaler les médailles ${ }^{25}$ ".

Établi le 7 novembre 1854, le règlement de la Société archéologique nous apprend que le conservateur des collections de la Société est assisté par une commission de trois membres nommés par le Comité central ${ }^{26}$. En outre, chaque année, le conservateur doit rendre compte à la Société des objets entrés au musée.

Le 21 octobre 1850, la Ville officialise cette quête du passé en créant une Commission municipale d'archéologie et charge la société de "rechercher les objets d'antiquité qui peuvent exister dans la ville, afin de sauver de la destruction et de les faire servir d'éléments à l'histoire locale dont l'absence se fait sentir". Les membres fondateurs de cette Commission sont les archivistes Louis-Jacques Bizeul et Jules Théodore Vandier, le baron Héracle Olivier de Wismes. Rapporteur de la Commission, Jules Théodore Vandier publie deux mois plus tard un premier état de l'archéologie nantaise ${ }^{27}$ et rend, en août 1855 , le premier rapport de cette Commission à la Ville de Nantes ${ }^{28}$.

Déjà, depuis 1847, certains des membres de la Société contribuent activement à l'archéologie locale, notamment Louis-Jacques Marie Bizeul qui est le premier à s'intéresser à Arthon-en-Retz et aux dolmens néolithiques (PortFessan, à Sainte-Pazanne, ou stèle de La Rudesse, à Bouaye). En 1851, il signale

23. Le premier Registre énonciatif, manuscrit tenu par Jules Théodore Vandier puis par Fortuné Parenteau, est conservé au musée Dobrée. Il couvre les années 1849-1868. Les œuvres sont inscrites dans ce registre par année et par ordre d'entrée.

24. Vandier établit un classement des objets qu'il publie en 1854, dans une "Notice sur la société archéologique de Nantes ", $R P O$, 1, février 1854, p. 246-265. Son classement comportait 10 grandes catégories : objets en terre, objets en pierre, objets en marbre, objets en plâtre, objets en bois, objets en métal, objets en verre et vitrifications, étoffes brodées, toiles peintes ou tableaux, enfin, plans, dessins, cartes et gravures. Il accompagne cette énumération d'une liste des donateurs dans laquelle on retrouve bon nombre de membres de la Société archéologique, comme Louis-Jacques Marie Bizeul, Louis de Becdelièvre, Joseph-Benjamin Bédert (ancien conservateur du musée des Beaux-Arts de Nantes), le baron Bertrand-Geslin, les architectes Chenantais (qui contribuera à l'édification du palais Dobrée), Driollet et Van-Iseghem, Frédéric Huette, Émile Pradal, ou Louis Prével.

25. VANDIER, Jules-Théodore, art. cit., p. 257. La méthode de classement dont il parle est celle conseillée lors du Congrès des sociétés savantes de Paris, février 1851; BSANLI, I, 1860, p. 478, 7 juin 1850 .

26. BSANLI, 1, 1859, p. 19-24, art. 14.

27. VANDIER, Jules-Théodore, Rapport sur les antiquités de Nantes, adressé au maire le 29 novembre 1850 et lu à la séance du 3 décembre 1850 : BSANLI, I, p. 72-88. Ce rapport fait un état des découvertes nantaises et des monuments encore existants par période et par secteur. Il désigne également les secteurs où des fouilles seraient indispensables.

28. Rapport sur les fouilles de Nantes, BSANLI, III, 1863, p. 83. 
la découverte de la voie romaine de Nantes à Montaigu, puis d'un camp celtique à proximité du lac de Grand-Lieu (île Dung) ${ }^{29}$. Son œuvre majeure reste, dès 1852 , son Histoire de Blain ${ }^{30}$, suivie d'un très important article publié entre 1859 et 1862 pour décrire l'essentiel des découvertes archéologiques locales ${ }^{31}$. Un autre sociétaire, Alfred-Victor-Benjamin d'Arondel, rédige dès 1846 une première Notice sur Rezé qui n'est malheureusement pas publiée mais seulement lue lors d'une séance de la Société. L'histoire de Rezé et les objets découverts ou recueillis par l'agent-voyer Sabot sont ensuite évoqués régulièrement à partir de 1852 par Vandier et Fillon ${ }^{32}$. Héracle-Olivier de Wismes, quant à lui, signale des découvertes importantes comme celle, à Nantes, d'un masque de théâtre en bronze (collection Mauduit, disparu) ${ }^{33}$. Comme Jules-Théodore Vandier, d'autres sociétaires cherchent à sauver les monuments nantais encore debout, comme le Bouffay ${ }^{34}$, ou ce qu'il en reste. À partir de 1855, les comptes-rendus des séances de la Société mettent en évidence le travail de ses membres et la place de cette société savante à Nantes en ce milieu du XIX ${ }^{\text {e }}$ siècle. On note d'ailleurs que 60 \% du budget de la Société sont alors dévolus à l'organisation des fouilles archéologiques.

Très rapidement, en raison de l'étroitesse de la salle de la rue du Moulin, la Société cherche des solutions pour abriter ses collections et la Ville propose de les installer au Muséum d'histoire naturelle, alors situé au Jardin des Plantes. Dans une séance du 28 mars 1854, la question du déménagement est évoquée :

"Relativement à la question d'un local à affecter au Musée archéologique et aux réunions de la société. $M$. Cuissand a exprimé l'intention de l'administration qui songe à réunir notre collection aux autres collections scientifiques et de lui donner à cet effet une place dans les bâtiments futurs du Jardin des Plantes. Là se trouverait également le lieu des séances mensuelles. Toutes les opinions protestent à ce projet. On répugne à voir la direction du Musée archéologique entre les mains d'un conservateur du Jardin des Plantes et on trouve peu praticable pour les membres de la société de les réunir de toutes les parties de la ville à ce point extrême, surtout pour l'hiver et avec la perspective de n'en sortir qu'à des heures fort avancées. "

Dans une séance de la Société archéologique du 25 avril $1854^{35}$, le problème est évoqué à nouveau :

29. BSANLI, II, 1862, p. 10, 5 août et 3 septembre 1851. C'est la première fois que l'on s'intéresse à l'archéologie du lac de Grand-Lieu. Les séances suivantes montrent qu'elle fait désormais partie des préoccupations de la Société archéologique.

30. BSANLI, II, 1862, p. 13, $1^{\text {er }}$ juin 1852.

31. Bizeul, Louis-Jacques Marie, "Des Namnètes aux époques celtique et romaine ", BSANLI, I, 1859, p. 72-88, 114-134, 151-169, 209-247; 1860, I, p. 276-313 et 335-370; 1861, I, p. 537-562 et II, 1862, p. 77-99 (avec pl.). Seule la seconde partie, sur la période romaine, a été publiée.

32. BSANLI, II, 1862, p. 14-15, 5 octobre 1852.

33. BSANLI, I, 1859, p. 183, 5 novembre 1847.

34. BSANLI, I, 1859, p. 183, 3 décembre 1847, et p. 189, 5 août 1848 .

35. Compte rendu de la séance du 25 avril 1854, dans les archives manuscrites de la Société archéologique de Nantes et de la Loire-Inférieure. Ces séances extraordinaires 
"Une discussion s'engage sur la translation du musée archéologique que la mairie se propose de faire construire au jardin des Plantes. Pour que cette translation eût lieu, il faudrait faire à l'administration municipale don des objets collectionnés au musée. Alors la direction en serait confiée au conservateur du musée d'Histoire naturelle, et la société y trouverait l'avantage de la gratuité d'un local convenable. Le musée Archéologique étant connu comme propriété communale, plusieurs collections particulières viendraient y affluer, et avant peu, il serait considérablement enrichi. Enfin, le musée serait ouvert à certains jours connus, et dès lors, les étrangers et amateurs sauraient où et quand ils pourraient en faire la visite. Ces avantages sont incontestables, mais ils sont accompagnés de graves inconvénients. D'abord, le musée ne doit pas être éloigné du lieu des séances de la Société, et, comme le dit le dernier procès verbal, la société ne pourrait se réunir au jardin des Plantes qui est situé à l'une des extrémités de la ville. D'un autre côté, la surveillance administrative serait peut-être insuffisante au point de faciliter les soustractions de certains objets. Cette crainte paraît fondée et le comité décide à l'unanimité qu'il convient de rester dans le statu quo, jusqu'à ce que l'administration ait pris une décision sur le musée d'histoire naturelle. Enfin, si la Société fait plus tard don du musée archéologique à l'administration municipale, la prudence lui conseillera sans doute de s'en réserver la direction et la surveillance. Cette solution ne sera finalement pas retenue, en raison de l'éloignement du Jardin public, et donc de la salle des séances de la Société, du centre ville ${ }^{36}$."

En 1856, devant l'ampleur des collections qui ne cessent de s'accroître - elles comportent plus de 800 numéros, soit près de 2000 objets -, la salle de la rue du Moulin doit être abandonnée. Vandier précise :

"Mais bientôt ce qu'on avait prévu arriva : les dons abondèrent et en peu de temps le local concédé fut rempli; c'est alors que [...] Monsieur le Préfet de la Loire-Inférieure, par un acte spontané de sa bienveillance, que le Conseil général s'empressa de sanctionner, nous ouvrit enfin les portes de l'Oratoire ${ }^{37}$. "

\section{La rédaction des catalogues et la conservation du musée de l'Oratoire, de 1856 à 1869}

Les collections sont transférées par les membres de la Société archéologique dans la chapelle de l'Oratoire ${ }^{38}$, local municipal restauré pour cet usage par le Conseil général de Loire-Inférieure, avec l'autorisation du préfet, Henri Chevreau. Fortuné Parenteau ne participe pas à ce transfert car la Société archéologique lui a confié la rédaction du catalogue du musée,

\footnotetext{
n'ont pas été retranscrites dans la publication a posteriori des comptes-rendus de séances, BSANLI, II, 1862.

36. Archives manuscrites de la Société archéologique, année 1854.

37. VANDIER, Jules Théodore, "Notice sur la Société archéologique de Nantes ", BSANLI, 1859 , I, p. $30 ; B S A N L I$, I, 1859 , p. 253 , $1^{\text {er }}$ septembre 1848 , démarches de Théodore Nau auprès du préfet pour l'affectation de la chapelle de l'Oratoire au futur musée archéologique; op. cit., p. 256-257, 5 janvier 1849.
}

38. Le transfert est terminé le 14 juin 1856. 
sans doute sur sa proposition ${ }^{39}$. Dans l'introduction au catalogue ${ }^{40}$, il précise que quinze jours seulement lui ont été accordés pour sa rédaction et son impression ${ }^{41}$. Il donne le plan du catalogue qui diffère sensiblement du classement établi par Jules Théodore Vandier : "Après en avoir fait la revue [des collections], nous avons cherché à le distribuer dans un plan où vinssent se grouper ensemble tous les objets d'une même nature ${ }^{42}$. "Il classe ainsi les objets par matériau et de façon chronologique (exemple : rubrique " monuments ", subdivisée en "à l'époque romaine " puis "à l'époque moderne "). Cette première démarche de classement est appelée à devenir une référence incontournable et marque les débuts d'une relative professionnalisation, très progressive, de l'archéologie. Outre les collections d'origine locale récoltées, notamment, par la Commission archéologique municipale, Fortuné Parenteau inscrit également à son catalogue les collections archéologiques égyptiennes (notices de Frédéric Cailliaud, pionnier de l'égyptologie puis directeur du Muséum d'histoire naturelle), grecques (céramiques ramenées par Olivier de Lauriston), ainsi que les premières collections ethnographiques d'Amérique du sud (vases péruviens de Jean-Baptiste Ténaud). Ce premier catalogue ne contient pas d'illustrations : le temps et l'argent ont dû manquer.

Le "Musée archéologique de Nantes" est inauguré le 14 juin 1856, à l'occasion de l'ouverture du XXIII ${ }^{\mathrm{e}}$ congrès de la Société française pour la conservation des monuments historiques. Le préfet de la Loire-Inférieure, Henri Chevreau et le baron de Girardot, secrétaire général de la Loire-Inférieure, participent à cette inauguration, ainsi qu'Arcisse de Caumont, archéologue et fondateur de la Société française d'Archéologie, et des membres de cette société. Dans son discours d'installation des séances de la Société dans les nouveaux locaux de la chapelle de l'Oratoire, son président, Théodore Nau, évoque les réalisations de la Société depuis sa création, et la "régénération de l'art par l'archéologie ${ }^{43}$ ".

Les seuls souvenirs qui nous restent de cette muséographie concernent l'installation des collections lapidaires. On n'a malheureusement aucune idée des vitrines qui devaient occuper le centre de la chapelle (fig. 6, 7, 8).

À l'occasion de cette inauguration, une importante exposition rassemble les principales collections privées nantaises à la chapelle de l'Oratoire, du 14 juin au 30 août $1856^{44}$. Il y a pratiquement autant de commissaires d'exposition que de prêteurs, le commissaire principal étant Théodore

39. "Nous avons pensé qu'un catalogue serait indispensable pour le visiter [le musée] ", écrit-il dans l'introduction du catalogue (voir note suivante), p. XI.

40. PARENTEAU, Fortuné, Catalogue et description des objets d'art du musée archéologique de Nantes et de la Loire-Inférieure, Nantes, 1856, imprimerie Armand Guéraud.

41. Parenteau, Fortuné, op. cit., 1856, p. XII.

42. Ibidem, p. XI.

43. BSANLI, III, 1863, p. 89, 6 janvier 1857.

44. Catalogue des objets exposés à l'Oratoire du 14 juin 1856 au 30 août 1856, dans les archives manuscrites de la Société archéologique de Nantes et de la Loire-Atlantique, 1856 . 


\section{Figures 6, 7 et 8 - Vues du musée archéologique dans la chapelle de l'Oratoire}

(fonds de la Société archéologique de Nantes et de la Loire-Atlantique, et MAÎTRE, L.éon, Les Villes disparues, I, p. 371)
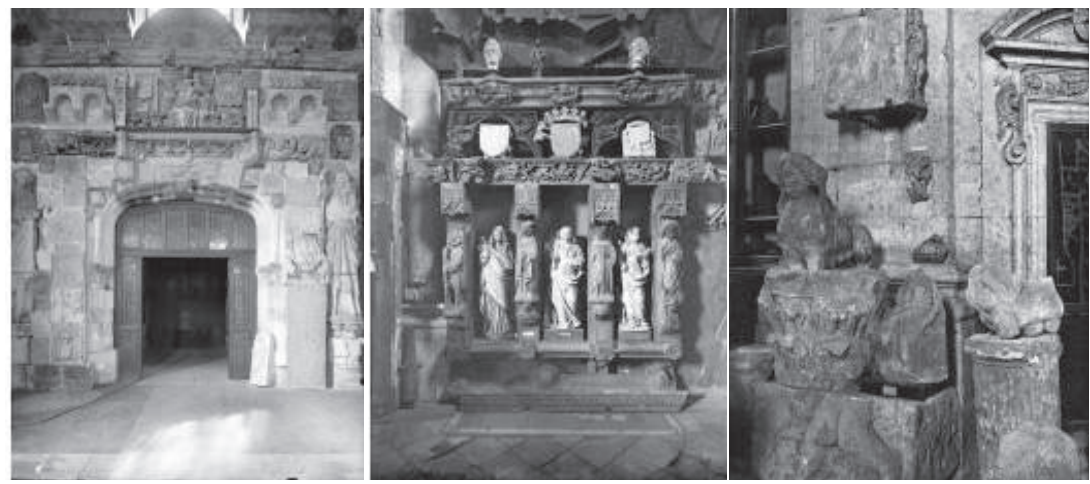

Nau, président de la Société. Soixante-quatorze prêteurs ont accepté de se dessaisir de leurs collections pour cette présentation. Les noms sont ceux des érudits nantais du milieu du XIX ${ }^{\mathrm{e}}$ siècle, tous membres de la Société archéologique : Charles Marie Bacqua, Frédéric Huette et son fils René, le docteur Aristide Mauduit, Frédéric Cailliaud et son père, Fortuné Parenteau (qualifié de "propriétaire "), Émile Pradal, Hubin de La Rairie, le baron Héracle Olivier de Wismes et Joseph Foulon sont les principaux prêteurs. On remarque que, à l'exception des collections égyptiennes de Frédéric Cailliaud, les cinq cents œuvres exposées touchent plutôt aux arts décoratifs qu'à l'archéologie. Cette exposition ne connaîtra pas le succès escompté : "La Société organisa une exposition dont le résultat ne répondit pas aux vues de ceux qui l'auraient désirée plus digne d'une grande cité et témoignant d'avantage du goût de ses habitants pour l'archéologie $e^{45}$. "

Dans le même temps et à l'occasion de cette réouverture, les publications archéologiques se multiplient. Fortuné Parenteau conforte sa réputation scientifique en rédigeant et illustrant de nombreuses publications : en 1856, "Les Médailles vendéennes ", dans la Revue des provinces de l'Ouest ${ }^{46}$; en 1859, dans le premier Bulletin de la société archéologique de Nantes, "Les fouilles de Pouzauges (Vendée), attributions gauloises ", avec planches, et un deuxième article intitulé "Fouilles archéologiques ${ }^{47}$ ", où il mêle des dessins d'objets du musée et d'autres de sa collection personnelle ${ }^{48}$.

45. BSANLI, I, 1860, p. 376.

46. BSANLI, 1856, p. 406-417.

47. BSANLI, I, 1859 , p. $192-247$ et p. 459-467.

48. $B S A N L I, \mathrm{I}, 1859$, p. 466 , pl. 2 . On remarquera notamment la très belle bague $\mathrm{n}^{\circ} 15$, en or et au chaton serti d'une émeraude, trouvée ou recueillie à Rezé par l'agent-voyer Sabot en 1854, et que Parenteau a attribué à sa collection personnelle. Il considère alors qu'il s'agit 
Parallèlement, Parenteau poursuit la constitution de ses collections : celle de bijoux tout d'abord, en se réservant les pièces les plus intéressantes lors des découvertes archéologiques, en achetant ou en échangeant, également, comme cela se pratiquait couramment à l'époque. Il prépare la publication de sa collection depuis $1858^{49}$ mais ne la publie qu'en 1878 dans son Inventaire archéologique précédé d'une introduction à l'étude des bijoux ${ }^{50}$ (fig. 9). Son autre collection importante est celle des monnaies. Après les monnaies féodales, il s'intéresse désormais aux monnaies gauloises qu'il collectionne et, converti par Bizeul de l'intérêt de ce monnayage, il publie un Essai sur les monnaies des Namnètes en $1862^{51}$.

On dispose aujourd'hui de nombreux carnets ou albums illustrés retraçant son parcours et ses hésitations : il revient fréquemment sur certaines attributions ainsi que sur certaines provenances d'objets de sa collection. C'est le cas pour la fibule étrusque en or de Saint-Philbert-de-Grand-Lieu (fig. 10 et cahier couleur, fig. 15), donnée comme trouvée dans une poterie avec des bracelets en or et des monnaies romaines impériales, puis, d'autres fois, achetée à un antiquaire nantais ${ }^{52}$ ! Ce qui, d'ailleurs, n'est peutêtre pas antinomique.

C'est sans doute à cette période que Pitre de Lisle du Dreneuc, qui sera son successeur, rencontre pour la première fois Fortuné Parenteau, à Nantes rue Mercœur, en achetant une gravure ${ }^{53}$. C'est pour lui l'occasion

d'une bague épiscopale ou abbatiale. Un peu plus tard, il enjolivera la découverte en l'attribuant à Adelphius, un évêque de Rezé au vI ${ }^{\mathrm{e}}$ siècle. Cette bague est aujourd'hui dans les collections du musée Dobrée grâce au don de la collection personnelle de Parenteau (1882).

49. Parenteau, Fortuné, 1858, Catalogue descriptif d'une collection de bijoux, musée Dobrée, fonds Parenteau, 1997, inv. 997.11.9, manuscrit.

50. PARENTEAU, Fortuné, 1878, Inventaire archéologique précédé d'une Introduction à l'étude des bijoux, 1878, Nantes, Forest et Grimaud, musée Dobrée, fonds Parenteau, inv. 997.11.18, 141 p. et $62 \mathrm{pl}$.

51. PARENTEAU, Fortuné, " Essai sur les monnaies des Namnètes ", BSANLI, II, 1862, p. 103124 (avec 3 pl.)

52. PARENTEAU, Fortuné, Catalogue descriptif d'une collection de bijoux, 1858 (manuscrit), musée Dobrée, fonds Parenteau, inv. 997.11.9, pl. VII et p. 173. Il décrit ainsi la découverte : "Fibule en or trouvée à Saint-Aignan près lac de Grand-Lieu (Loire-Inférieure) ; travail très fin, filigrane et appliques de petites violettes d'or. La découverte comprenait aussi une magnifique paire de bracelets en or [qu'il n'a jamais dessinés malheureusement] d'une conservation irréprochable, composés d'une grosse torsade de tige d'or terminée par un bouton et s'agrafant dans un anneau; ils pesaient 190 grammes d'or fin; achetés par M. Rousseau, ils ont été s'enfouir dans une collection de la capitale. Je n'ai pas cru devoir en faire l'acquisition. "Dans un autre manuscrit (musée Dobrée, fonds Parenteau, inv. 997.11.7), il ajoute "la petite fibule d'or de travail étrusque, ornée de grenetis et de violettes d'or en relief, a été rencontrée avec deux cents monnaies consulaires d'argent dans un vase rouge à Saint-Aignan près du lac de Grand-Lieu (Loire-Inférieure). Le vase renfermait aussi une admirable paire de bracelets d'or composés de filets torsadés réunis par une maille et un bouton, près de quatre cents fr. d'or comme valeur intrinsèque. Je refusais bien à tort d'en faire l'acquisition. Tous ces bijoux étaient de provenance étrusque, et contemporains de la conquête romaine".

53. Pitre de Lisle qualifie les étalages de la rue Mercœur de "petite Bourse des archéologues nantais " : RBV, LII, 1882, p. 469. 
Figure 9 - Objets archéologiques provenant de Rezé (Fortuné Parenteau, 1859, musée Dobrée, fonds

Parenteau, inv. 997.11.17, p. 11, Pl II - cl. musée Dobrée)

\section{Figure 10 - Fibule étrusque en or de Saint-Aignan-de-Grand-Lieu}

(Fortuné Parenteau, musée Dobrée, inv. 882.1.443, dans Catalogue descriptif d'une collection de bijoux, 1858, dessin aquarellé, fonds Parenteau 997.11.9, p. 19, pl. VIIcl. musée Dobrée)
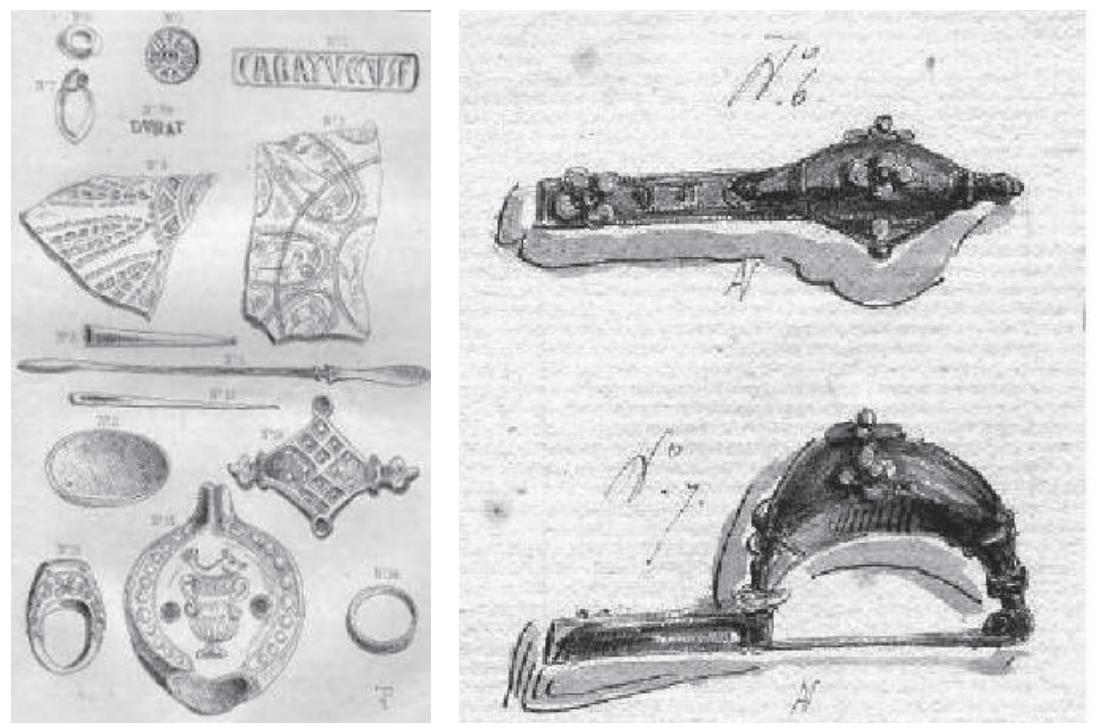

de visiter le " cabinet » de Parenteau dont il fait une savoureuse description qui nous éclaire sur l'éclectisme des goûts du collectionneur :

"Pour un archéologue à ses débuts, le cabinet de M. Parenteau était plus qu'une collection d'antiquités; c'était l'ARCHÉOLOGIE vivante et parlante. Les panneaux étaient couverts d'armes et de parures de toutes les époques et de tous les pays; ça et là, des œuvres d'art, des faïences richement nuancées, égayaient cet arsenal cosmopolite. Au fond, bien en face du jour, se dressait la bibliothèque en bois d'ébène, soigneusement voilée de rideaux verts. La première fois que je vis s'ouvrir les larges battants de ce sanctuaire, je crus avoir sous les yeux les trésors d'Aladin. Une incroyable profusion de bijoux, suspendus à des cordons de soie, couvrait toutes les parois du meuble : les bracelets d'or, les grands colliers gaulois, les perles des dolmens, les anneaux des chevaliers romains, brillaient au premier rang. Des bronzes, enroulés comme des serpents et couverts d'une patine glacée, se détachaient sur le ton fauve du vieil or; puis venaient d'interminables séries d'agrafes, de fibules, de plaques incrustées ou niellées, d'émaux aux vives couleurs. Toutes les merveilles d'orfèvrerie du Moyen Age et de la Renaissance étaient là représentées. Chaque objet de cette 
collection, étudié avec un soin passionné qui ajoutait encore à sa valeur, servait à établir d'ingénieux rapprochements entre les différentes périodes de l'art et de l'industrie. [...] Alors, de ces comparaisons jaillissaient des aperçus soudains, qui éclairaient dans le passé la filiation de notre art national. Ce n'était plus un objet que l'on avait sous les yeux, c'était une époque toute entière, avec ses caractères principaux, son origine et ses transformations. [...] Il aimait, avant tout, à initier, abandonnant de grand cœur les trésors d'érudition et de science qu'il avait accumulés pendant près de quarante années d'études ${ }^{54}$."

La participation de Fortuné Parenteau à l'archéologie locale semble toutefois limitée. On peut définir le personnage comme un «archéologue antiquaire " ou, comme le fait Anatole de Brémond d'Ars en parlant de ses disciples archéologues dans son allocution comme nouveau président de la Société archéologique en 1884, d' " amateurs d'antiquités ", même si, dans ce même discours, il parle du regretté Parenteau en tant que "chercheur infatigable, qui savait si bien dans quelles poussières se rencontrent les heureuses trouvailles ${ }^{55}$ ". Les comptes-rendus de séances de la Société font fréquemment état de divergences d'interprétation sur les sites archéologiques, notamment entre Parenteau et Bizeul ${ }^{56}$. On constate également que, jusqu'en 1860, la Société accepte le principe de l'échange des collections du musée : certains membres reprennent des collections et en proposent d'autres à leur place ${ }^{57}$.

À la fin de 1858, Jules Théodore Vandier, très fatigué, demande à se retirer de la direction du musée archéologique de la Société. Il aura tenu l'inventaire manuscrit des collections de la Société jusqu'à son départ ${ }^{58}$. Le travail de Fortuné Parenteau porte ses fruits car il est nommé archiviste de la Société puis premier conservateur du musée en janvier $1859^{59}$. Il prend alors en charge l'inventaire manuscrit des collections tandis que la Société décerne à Vandier le titre de conservateur honoraire ${ }^{60}$, peu avant sa mort en 1861. Le faible budget alloué au musée ne permet d'entretenir

54. LiSLE DU Dreneuc, Pitre de, RBV, LII, 1882, p. 470-471.

55. BRÉMOND D'ARS, Anatole de, BSANLI, 23, 1884, p. XXIV.

56. Par exemple, BSANLI, I, p. 91-92, 5 juillet 1859 : opposition entre Bizeul et Parenteau au sujet d'un site gallo-romain à côté de Redon; là où Parenteau voit une villa galloromaine avec des fours de potiers, Bizeul ne voit que des hypocaustes.

57. 29 décembre 1857, manuscrit de la Société archéologique.

58. "Catalogue énonciatif du musée d'archéologie ", manuscrit, musée Dobrée : le dernier numéro inscrit par Vandier est le n 1010 et concerne 6 objets découverts à Rezé.

59. PARENTEAU, Fortuné, Catalogue complet de ma collection archéologique, $1^{\mathrm{er}}$ janvier 1881, manuscrit, musée Dobrée, fonds Parenteau, inv. 997.11.5, introduction, p. 3 : «J'avais été chargé par la Société archéologique de diriger le musée en remplacement de Monsieur Vandier très fatigué et cela à l'unanimité des membres du Comité. "L'élection de Fortuné Parenteau comme conservateur du musée départemental d'Archéologie sera confirmée par l'arrêté préfectoral du 2 janvier 1861.

60. BSANLI, I, 1859, p. 31-32, 11 janvier 1859 : «Sur la proposition du Président [Théodore Nau ], la Société décerne à l'unanimité, à M. Vandier, ancien conservateur, le titre de conservateur honoraire, en reconnaissance du dévouement et du zèle intelligent qu'il a constamment montré dans l'accroissement et la prospérité du musée. " 
ni les bâtiments ni les collections ${ }^{61}$. Face à cette conjoncture défavorable, la Société propose de faire le don de ses collections au Conseil général de la Loire-Inférieure :

«Le président [Théodore Nau] rappelle quelles furent les origines de la Société et ses vicissitudes avant de venir occuper le local qui renferme son musée, et dont on ne saurait regarder la possession comme définitive tant que ce musée ne sera considéré que comme une collection privée, que nul n'a intérêt à protéger et à développer. Il invite en conséquence ses collègues à examiner s'il ne conviendrait pas de faire auprès de l'Administration une démarche tendant à ce que le musée soit considéré comme une propriété du Département. La Société, considérant tout l'avantage qui en résulterait pour l'accroissement du musée, et la possibilité de revendiquer dès lors une foule d'objets curieux qui tendent à disparaître; considérant encore qu'une fois en possession du musée, le Département ne pourra manquer de prendre à tâche sa restauration et son développement; émet, à l'unanimité, le vœu que M. le Préfet accueille favorablement la demande qui va lui être adressée de considérer le musée comme musée départemental, et lui en accorde le titre ${ }^{62}$. "

Lors de la séance du 6 novembre $1860^{63}$, le président Nau annonce l'acceptation du don par le Conseil général (session d'août 1860) ${ }^{64}$ : le musée devient donc départemental et le Conseil général vote les fonds nécessaires à la restauration de la façade de la chapelle de l'Oratoire. C'est Gustave Bourgerel, l'architecte départemental, qui en est chargé et qui fera achever les sculptures. Sous l'escalier monumental donnant accès à la chapelle, il place le logement du concierge, "le fidèle Thomas ", gardien du nouveau musée ${ }^{65}$.

L'année suivante, par un arrêté du 19 décembre 1860, le préfet de LoireAtlantique charge MM. de Kersabiec, Bacqua et Bourgerel de procéder à l'inventaire des objets du musée donnés au Département. La remise des objets sera faite par MM. Nau, Huette, Parenteau et Martineau. Le procès verbal du 27 décembre 1860 entérine le don des objets ${ }^{66}$ et Parenteau date du 28 décembre 1860 l'Inventaire des collections cédées par la Société archéologique au Département, arrêté au numéro $1040^{67}$. L'arrêté du 2 jan-

61. Les frais d'entretien et d'aménagement du musée étaient beaucoup trop lourds pour la Société archéologique qui comptait alors une centaine de membres donnant une cotisation annuelle de 10 francs.

62. BSANLI, I, 1860, p. 178, 5 juin 1860, et musée Dobrée, fonds Parenteau, inv. 997.11.38, p. 67, note manuscrite de Fortuné Parenteau sur cette séance.

63. BSANLI, I, 1860, p. 316, 6 novembre 1860.

64. Procès-verbal de la séance du $1^{\mathrm{er}}$ septembre 1860 : «La Société archéologique, par une délibération du 5 juin 1860, fait offre au département du Musée d'archéologie et demande qu'à l'avenir, ce musée prenne le titre de Musée départemental ": " Musée départemental d'Archéologie ", La Ville de Nantes et la Loire-Inférieure, Nantes, impr. Émile Grimaud, 1898 , p. 75.

65. LISLE DU Dreneuc, Pitre de, « M. Fortuné Parenteau, conservateur du musée de l'Oratoire de Nantes ", $R B V, 1883$, p. 143. Le gardien du musée restera en poste 25 ans jusqu'à la mort de Parenteau dont il était devenu l'ami.

66. BSANLI, I, 1861, p. 371-372, 8 janvier 1861.

67. Bibliothèque du musée Dobrée. En réalité, cet inventaire est le catalogue du musée dressé en 1856 par Fortuné Parenteau. Il est annoté par le vicomte de Kersabiec qui a fait 
vier 1861 nomme Fortuné Parenteau conservateur du musée départemental d'Archéologie, sans indemnité ${ }^{68}$. Le conservateur poursuivra de la même manière et dans le même cahier l'inscription manuscrite des novelles acquisitions au fur et à mesure de leur entrée dans le nouveau musée départemental d'Archéologie et ce, jusqu'au $1^{\text {er }}$ avril 1881, date à laquelle, sans doute trop fatigué, il cédera la charge de l'inventaire à Pitre de Lisle du Dreneuc, au numéro 1306.

En juillet 1862, Fortuné Parenteau complète l'inventaire des collections de la Société archéologique par le don des dessins, gravures, lithographies, photographies appartenant à la Société qui en achète d'autres au baron Héracle Olivier de Wismes, pour le musée départemental :

« Le vingt six juin mil huit cent soixante deux, par décision du Comité central, la Société archéologique a fait l'acquisition au prix de trois cents francs, d'un certain nombre de dessins originaux, gravures, etc., le tout appartenant à M. le baron de Wismes. Monsieur Stéphane de La Nicollière a été, entre la Société et M. de Wismes, l'intermédiaire de cette transaction. Tous ces dessins se rapportent à l'histoire de Nantes à la fin du XVIII siècle et plusieurs d'entre eux représentent des monuments aujourd'hui disparus. La

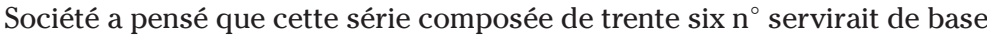
à une collection plus importante en la joignant aux dessins que la Société possède déjà ou qu'elle pourra se procurer un jour ${ }^{69}$. "

À la demande du baron Auguste Théodore de Girardot, secrétaire général du Département de la Loire-Inférieure, une Commission administrative est chargée de veiller au bon fonctionnement du musée. Elle est nommée en janvier 1861 pour "prendre les intérêts du musée, pour veiller à son accroissement, à la conservation des objets qu'elle renferme, à l'emploi des subventions qui pourraient lui être accordées, etc. ${ }^{70}$ ". Le préfet en nomme les membres qui appartiennent au Conseil général et à la Société archéologique : Auguste Théodore de Girardot en est le président. Il est assisté de Charles Marie Bacqua, conseiller général, Louis Jacques Marie Bizeul, conseiller général et président d'honneur de la Société archéologique, l'abbé Félix Fournier, curé de Saint-Nicolas et vice-président de la Société archéologique, Émile Gautier, secrétaire adjoint de la Société archéologique, Frédéric Huette, trésorier de la Société, Édouard Sioc'han de Kersabiec, conseiller de Préfecture, secrétaire général de la Société archéologique,

insérer entre chaque page des feuilles blanches pour y porter de sa main la valeur des objets et son paraphe et qui précise la méthode adoptée : "Nous nous sommes transportés devant chaque objet inscrit au catalogue et après vérification faite de son existence et de son état de conservation, nous l'avons accepté, au nom du Département et le président a, pour la Commission, apposé son paraphe au devant de chacun d'eux. "Aux 865 numéros du catalogue de 1856, il ajoute un Supplément qui va jusqu'au numéro 1040 ainsi que quelques monnaies. La valeur globale du don est estimée à 15882 francs.

68. BSANLI, I, 1860, p. 372, 8 janvier 1861.

69. Musée Dobrée, Notes pour le $3^{\mathrm{e}}$ catalogue, du $1^{\text {er }}$ juin 1869 à fin 1901, Registre énonciatif, manuscrit, p. 156.

70. BSANLI, I, 1860, p. 372, 8 janvier 1861. " Musée départemental d'Archéologie ", La Ville de Nantes et la Loire-Inférieure, 1898, II, p. 75. 
Théodore Nau, président de la Société archéologique et, enfin, Fortuné Parenteau, le conservateur du musée. En compensation du don de ses collections au Département, la Société archéologique de Nantes obtient une salle de réunion dans la chapelle de l'Oratoire pour la tenue de ses séances et le conservateur du musée archéologique est présenté par la Société à la nomination du préfet ${ }^{71}$.

Pour marquer ce don, un grand projet d'exposition archéologique est lancé par Charles Bacqua au sein de la Société pour faire le pendant à l'exposition industrielle et artistique projetée à Nantes en juillet 1861 . Malheureusement, le projet, qui a donné lieu à de vives discussions au sein de la Société ${ }^{72}$, avorte et la Société, échaudée sans doute par le peu de succès de sa précédente exposition de 1856, peu satisfaite, semble-t-il aussi, du don au Département de ses collections, semble alors très désabusée :

"Le Musée, propriété toute nouvelle du Département, est une propriété tout à fait inconnue de la plupart de ceux qui ont accepté le don. Ils savent qu'il existe, qu'il renferme de vieux débris d'une valeur et d'une importance qu'on est, malheureusement, trop porté à contester; mais ils ne sont pas encore entrés dans le détail des objets qui y sont réunis. Or, dissimuler ces objets sous des ornements d'emprunt, placer un bahut soigneusement travaillé auprès d'une pierre grossière de l'époque romaine, ne serait-ce pas vouloir confisquer l'attention et faire disparaître le Musée, tel qu'il a été donné, tel qu'il se comporte réellement? Ne serait-ce pas vouloir ranimer la discussion sur de prétendues richesses qui ne tendraient qu'à dissimuler sa pauvreté, pour faire retour ensuite aux mains de ceux qui les auraient déposés ${ }^{73}$ !

Jusqu'en 1876, on ne connaît pas les travaux de la Commission administrative du musée départemental d'Archéologie. Certains comptes-rendus de séances de la Société archéologique nous apprennent toutefois que les relations sont parfois tendues entre le Conseil général et la Société et tournent parfois au règlement de compte. Ainsi, en 1868, Édouard de Kersabiec, le président de la Société, lit un article de l'Année Illustrée ${ }^{74}$, consacré à " Nantes et la Loire-Inférieure », dans lequel il est fait état du « Musée archéologique, fondation du baron de Girardot ". La réaction des membres de la Société est vive :

"Si M. le baron de Girardot a le goût des collections archéologiques [...], Il n'en est pas moins vrai qu'il n'a coopéré en rien à la fondation du Musée archéologique [...] La vérité est que le musée a été fondé et s'est accru, grâce uniquement à l'initiative, aux dons et à la persévérance des membres de la Société Archéologique, qui l'a offert depuis au Département, lequel l'a accepté de ses mains ${ }^{75}$."

71. Règlement du musée archéologique de Nantes, s.d. (1888?), signé par Charles Marionneau et Paul Eudel, document préparatoire au règlement intérieur de 1889, repris en 1894, archives du musée Dobrée.

72. BSANLI, I, 1860, 5 mars 1861, p. 374, et 9 avril 1861, p. 375-378.

73. BSANLI, I, 1860, p. 377.

74. Année illustrée, $\mathrm{n}^{\circ} 21$, jeudi 7 mai 1868.

75. BSANLI, 8, 1868, p. 96-97, 19 mai 1868. 
Cette même année la Commission administrative du musée refusera à la Société archéologique les subsides demandés pour les fouilles du lac de Grand-Lieu ${ }^{76}$.

Fortuné Parenteau, quant à lui, assure avec constance la conservation du musée, quoique la subvention départementale ne permit au musée que de vivre très chichement et interdise tout développement. Malgré tout Parenteau poursuit son enrichissement comme nous le décrit Pitre de Lisle du Dreneuc :

" Il consacra tous ses soins et ses plus ardentes recherches à développer l'établissement qui lui était confié. Le Musée était peu important quand il en prit la direction, et le budget destiné à l'accroissement des collections, complètement insuffisant. Cependant, grâce à une administration sage et bien réglée, grâce aussi à de généraux secours, puisés dans la bourse du Conservateur, le Musée s'accrut d'année en année; de belles pièces, de riches découvertes vinrent remplir les vitrines de l'Oratoire et lui valurent un des premiers rangs parmi les Musées archéologiques de France ${ }^{77}$. "

\section{Parenteau lui-même décrit son rôle en 1868 :}

"Le Musée de l'Oratoire n'est plus à l'état d'embryon et [...] le musée de la Société Archéologique, dédaigné de tous, est devenu une institution départementale, le musée de la contrée et du comté nantais [...]. Maintenant nous sommes bien pauvres : le luxe est banni de nos salles, nos murs sont dénudés, nos vitrines sont mesquines [...]. Au milieu de ces débris, de ces richesses, mon rôle est tout tracé. Pionnier de la science future, tenant d'une main une pioche et une lanterne de l'autre, je ne dirai pas drapé, mais à peine couvert de mon manteau troué, je fouille le sol où s'élevèrent nos monuments effacés. Je recueille pour vous des débris, je tâche de les faire parler, moi qui ne suis pas un savant, mais un homme de bonne volonté ${ }^{78}$."

Parenteau suit, sans y participer, les importantes découvertes locales et régionales, comme celles des dépôts de l'âge du Bronze de Port-SaintPère (Loire-Atlantique) (fig. 11), en 1851, de Questembert (Morbihan) (fig. 12 et cahier couleur, fig. 16), en $1863^{79}$, et de Plouguerneau, près de Brest (Finistère), dont il va dessiner certains objets qui entrent dans les collections du musée archéologique ou dans sa propre collection. Comme nombre de collectionneurs de cette époque, il piste, pour sa propre collection, les objets sortis des fouilles (cahier couleur, fig. 17), les plus belles pièces étant généralement vendues aux antiquaires parisiens ou locaux. Le dépôt de Saint-

76. BSANLI, 8, 1868, p. 172,5 août 1868 .

77. LISLE Du DRENEuc, Pitre de, « M. Fortuné Parenteau, conservateur du Musée de l'Oratoire à Nantes ", RBV, 1883, p. 142.

78. Parenteau, Fortuné, Le Fondeur du Jardin des Plantes de Nantes et son confrère de Rezé, Nantes, 1868, p. 15.

79. PARENTEAU, Fortuné, planches diverses destinées à l'Inventaire archéologique de Parenteau, 1878, musée Dobrée, fonds Parenteau, inv. 999.11.17. Fortuné Parenteau acquiert cette pièce en 1869, "trouvée avec des haches ", provenant du dépôt de Questembert découvert en 1863 et dont quelques exemplaires ont certainement circulés chez les antiquaires et orfèvres régionaux. Il conserve dans sa collection personnelle la bague en or trouvée dans le dépôt. 
Figure 11 - Planche du dépôt de Saint-Père-en-Retz

(Fortuné Parenteau, Inventaire archéologique, 1878, musée

Dobrée, fonds Parenteau, inv. 997.11.4, p. 276 - cl. musée Dobrée)
Figure 12 - Boîte de moyeu de roue, dépôt de Questembert (Morbihan)

(inv. 882.1.301, dessin aquarellé, F. Parenteau, musée Dobrée, fonds Parenteau, inv. 997.11.17, p. 129 cl. musée Dobrée)
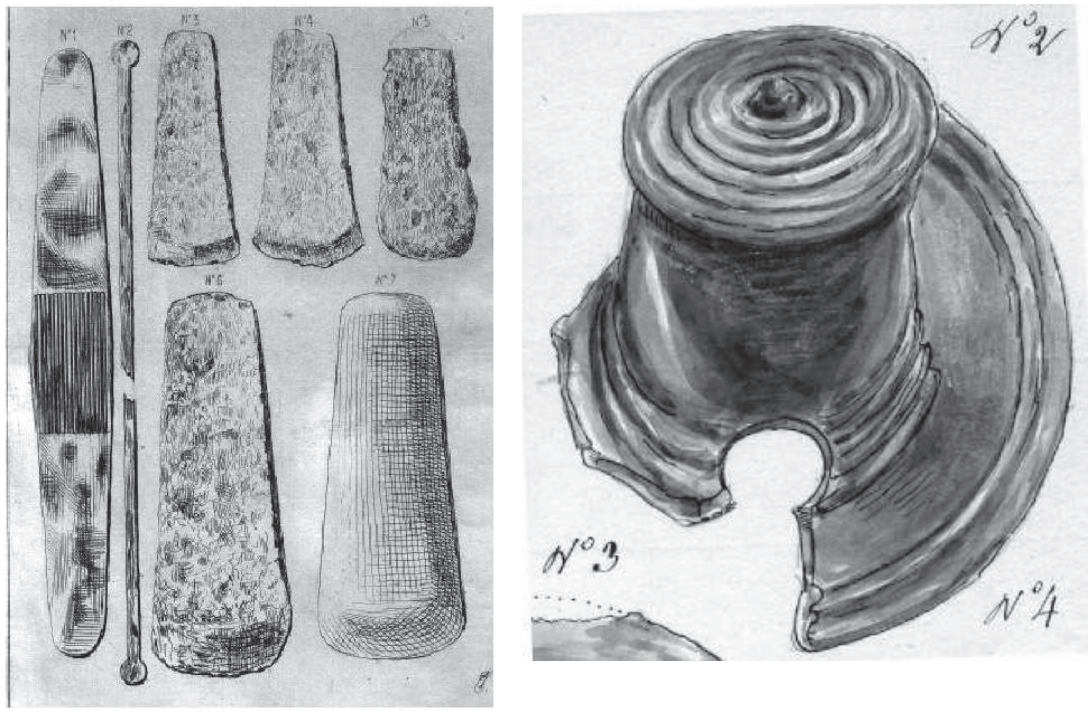

Père-en-Retz est ainsi dessiné dans son Inventaire archéologique de 1878, en précisant dans son descriptif que la bague en or trouvée avec le dépôt est entrée dans sa collection personnelle après achat à un orfèvre ${ }^{80}$ (fig. 13); le très beau bracelet en or du type gargantilla, trouvé dans le dépôt, est malheureusement parti chez un orfèvre parisien. Il est aujourd'hui perdu.

Au mois de juillet 1863, Parenteau se passionne pour la découverte d'un " atelier de fondeur " à Rezé trouvé en même temps qu'un laraire, à l'occasion de la reconstruction de la nouvelle église Saint-Pierre ${ }^{81}$. Il publie cette découverte en $1868^{82}$ (fig. 14 et 15) et, curieusement, ne comprend pas

80. Musée Dobrée, fonds Parenteau, inv. 997.11.4, p. 247 et 249 : " Sous un Dolmen de St-Père-en-Raiz, Loire-Inférieure, on découvrit au mois de mai ou de juin 1851, un diadème ou bandeau d'or [...], un ruban terminé par deux petits disques et deux haches celtiques en cuivre très primitives. Ces curieux objets restèrent plus d'un mois chez un orfêvre de la rue Contrescarpe à Nantes, puis furent emportés et vendus à Paris [...]. Plus tard, la bague en or de ma collection, bagues à crochets avec filets arriva chez les orfêvres de Nantes. Elle provient de la même découverte. "

81. Parenteau, Fortuné, Notes diverses, musée Dobrée, fonds Parenteau, inv. 997.11.16, p. 27-28.

82. Parenteau, Fortuné, Le Fondeur du Jardin des Plantes de Nantes et son confrère de Rezé, Nantes, V. Forest et E. Grimaud, 1868, p. 16-19. Sur la planche concernant l'atelier de 
Figure 13 - Bague en or du dépôt de Saint-Père-en-Retz (musée Dobrée, inv. 882.1.437, dessin de F. Parenteau, musée Dobrée, fonds Parenteau,

Catalogue complet de ma collection archéologique, 1 er janvier 1881, inv. 997.11.5, p. 5 cl. musée Dobrée)

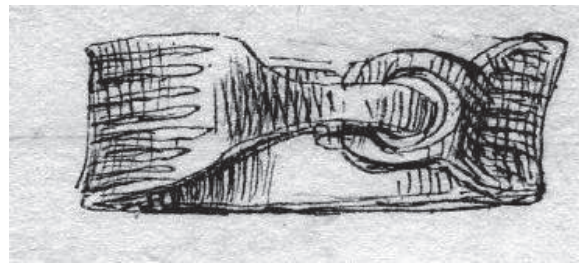

tout de suite l'importance de la découverte du laraire auquel il ne prêtera attention que plus tardivement, lors de la publication de son Essai sur les poteries de l'Ouest. En revanche, il prend le soin de dessiner, sur place, les fouilles de Rezé en 1863 et 1864 (cahier couleur, fig. 18), ainsi qu'un croquis du laraire (sans le porc) et le porc tout seul (fig. 16 à 18 et cahier couleur, fig. 19). En 1865, il publie l'Essai sur les poteries antiques de l'Ouest de la France, petit opuscule illustré de planches qui sont celles qu'il a préparées et fait graver, quatre ans auparavant, pour la seconde édition du Catalogue du musée archéologique de $1869^{83}$. Il y traite de quelques céramiques peu courantes (lampions, chenets, fragments de construction, briques historiées mérovingiennes, etc.), mais aussi du laraire de Rezé dont c'est la première publication avec sa planche.

Malheureusement, Parenteau ne suit pas les fouilles des fossés du château de Nantes où disparaissent les derniers pans de la muraille : "L'un des derniers jalons qui servaient à rattacher à la vieille forteresse, les murailles entourant jadis la Motte Saint-Pierre. Il est malheureux de voir ainsi s'effacer les traces de cette enceinte ", souligne Aristide Van Iseghem dans une séance de la Société archéologique ${ }^{84}$.

En 1863 également, le baron Auguste de Girardot, président de la Commission administrative du musée, fit la demande auprès de l'État d'un dépôt de céramiques grecques et étrusques de la collection Campana, provenant du musée de Napoléon III à Paris. Les musées de province bénéficiaires de ces dépôts furent classés en trois catégories et le musée archéologique de Nantes eut la chance de faire partie des vingt-et-un musées de première classe. En conséquence, il reçut un dépôt de quatre-vingt-dix-sept pièces (en réalité cent une). La presse locale se fit l'écho de ce dépôt précieux pour le musée ${ }^{85}$ malgré une petite aventure rapportée dans une séance de la Société par Fortuné Parenteau :

Rezé, il mêle les objets de la découverte avec des rouelles en or d'une autre provenance. Il conserve le moule à rouelles dans sa propre collection.

83. Parenteau, Fortuné, Essai sur les poteries de l'Ouest de la France, Nantes, Henri Charpentier éd., 1865,22 p. et $5 \mathrm{pl}$.

84. BSANLI, II, 1863, p. 79, 14 avril 1863.

85. L'Union bretonne, 2 mars 1863 : il est fait état d'un dépôt de 88 objets et il est précisé que "Nantes est l'une des cités les mieux traitées dans le partage du musée Campana ». 
Figure 14 -Moule en schiste pour rouelles en bronze

(musée Dobrée, inv.56.6068, dessin aquarellé de Fortuné Parenteau, musée Dobrée, fonds Parenteau, inv. 997.11.16, p. 262 - cl. musée Dobrée)
Figure 15 - Mobilier de l'atelier du fondeur de Rezé

(Fortuné Parenteau, Le fondeur du jardin des Plantes de Nantes et son confrère de Rezé; attributions celtiques et gallo-romaines, Nantes, 1868, musée Dobrée, fonds Parenteau, inv. 997.11.10 [3], p. 67, pl. III - cl. musée Dobrée).

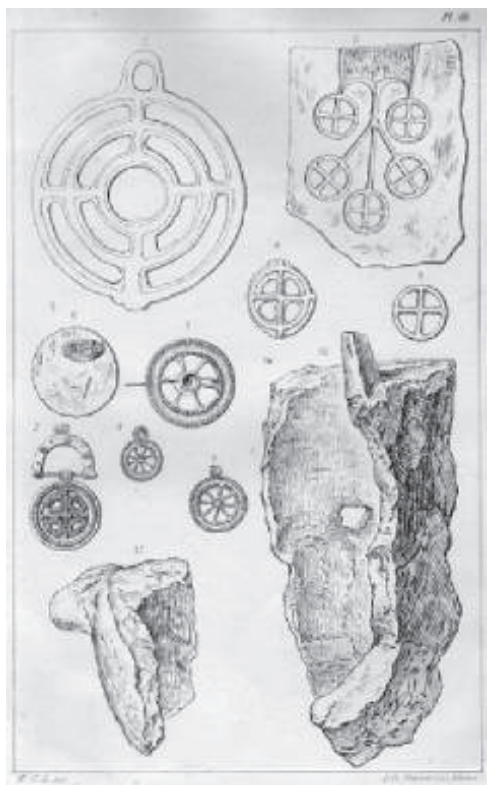

"Les objets adressés à Monsieur le Maire de Nantes ont été transportés au musée des Tableaux [des Beaux-Arts], déballés et installés comme propriété municipale, et il a fallu l'intervention du Ministre d'État pour que le Musée archéologique n'en fût pas dépossédé, l'Administration municipale et la Commission du Musée de Peinture tenant fort à ne s'en point dessaisir, alors qu'elles n'avaient fait aucune démarche pour les obtenir ${ }^{86}$."

Même s'il reconnaît que «le musée n'eut pas lieu de se plaindre dans la distribution qui fut faite ", Fortuné Parenteau n'apprécia guère bon nombre des objets de cet envoi ${ }^{87}$.

En 1868, Parenteau se passionne de nouveau pour les trouvailles de dépôts métalliques de l'âge du Bronze. Cette fois-ci, il s'agit de la décou-

86. BSANLI, II, 1863, p. 77, 14 avril 1863.

87. Parenteau, Fortuné, Catalogue du musée archéologique de Nantes, Nantes, 1869, p. 19. 
Figure 16 - Le laraire de Rezé (L.A.) à sa découverte en juillet 1863

(Fortuné Parenteau, musée Dobrée, fonds Parenteau, inv. 997.11.39, p. 67 - cl. musée Dobrée)

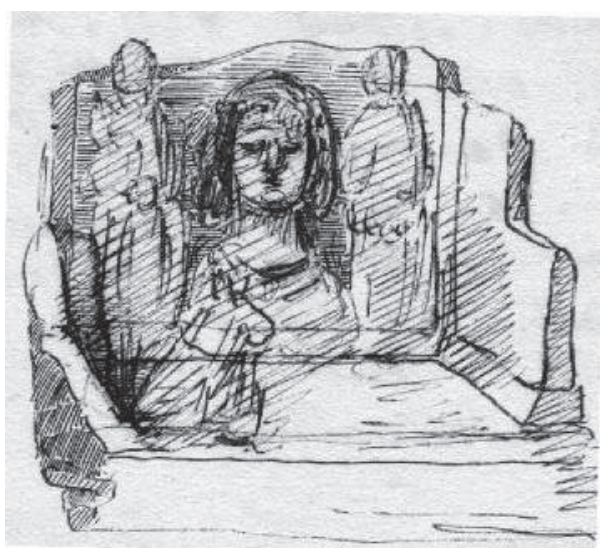

Figure 17 - Le laraire de Rezé (L.-A.), restitution gravée

(Fortuné Parenteau, Essai sur les poteries antiques, 1865, p. 29, pl. 3 cl. musée Dobrée)

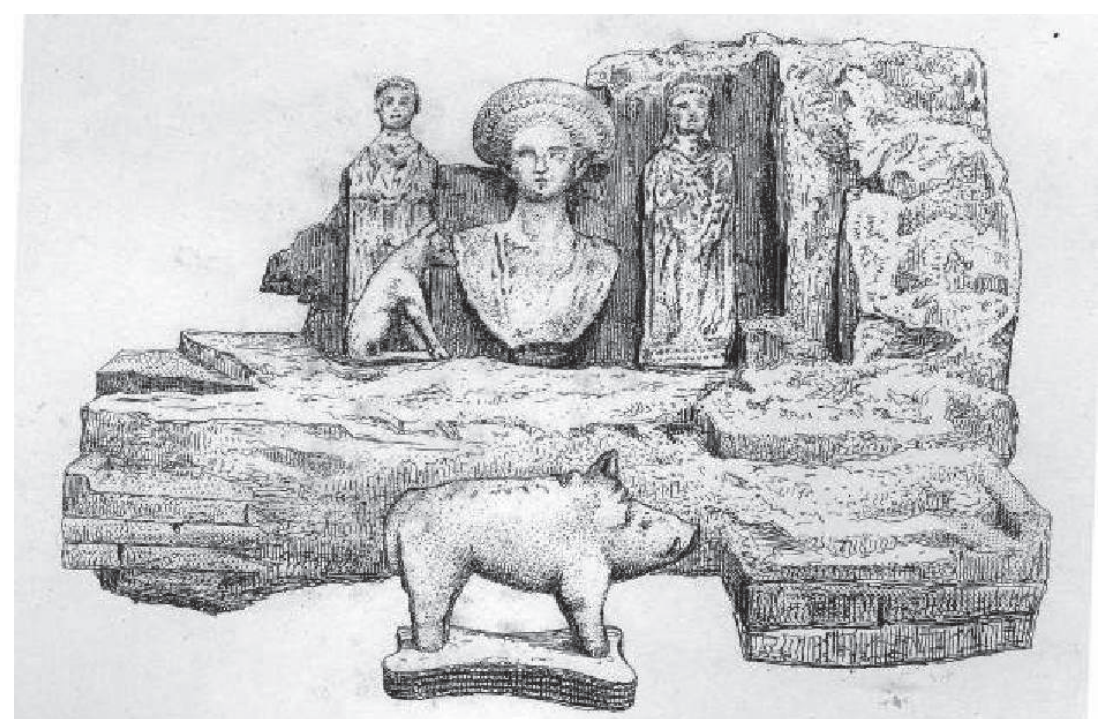

verte, en décembre $1867^{88}$, de ce qu'il appelle "le fondeur du jardin des Plantes " de Nantes et qu'il publie avec l'atelier de Rezé découvert cinq ans auparavant ${ }^{89}$. À cette occasion, et pour la première fois, il fait analyser à

88. BSANLI, VII, 1867, p. 229, 17 décembre 1867.

89. PARENTEAU, Fortuné, "Découverte du Jardin des Plantes de Nantes ", BSANLI, VIII, 1868, p. 19-49; idem, Le Fondeur du Jardin des Plantes de Nantes et son confrère de Rezé, Nantes, V. Forest et E. Grimaud, 1868, 32 p. et 3 pl. de W. C. Lukis. 


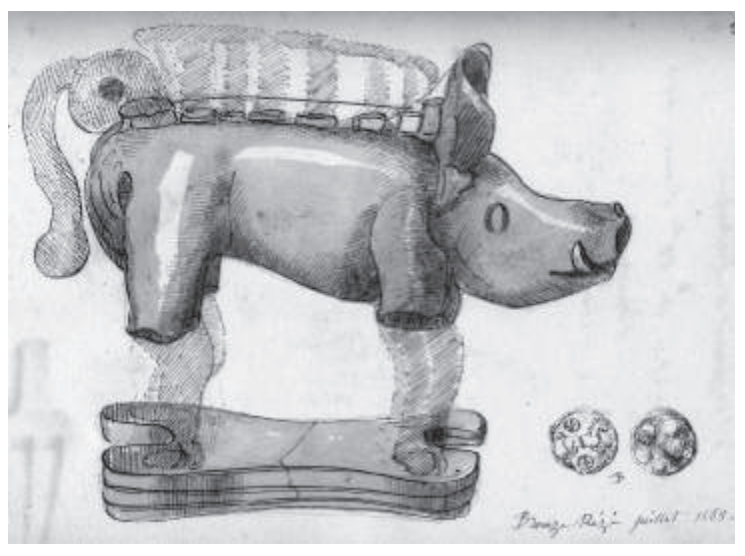

Figure 18 -

" Restitution du porc du laraire de Rezé et Bronze Rézé, juillet 1863 " (Fortuné Parenteau, musée Dobrée, fonds Parenteau, inv. 997.11.39, p. 57 cl. musée Dobrée)

l'École impériale des Mines quelques objets en bronze de cette découverte. S'agissant de 155 objets brisés, il pense être en présence d'un "fondeur ambulant " qui a recueilli du matériel appartenant à " l'équipement militaire des Armoricains ${ }^{90} "$. Enfin, il explique que le développement de cette métallurgie locale n'a pu se faire que grâce à la présence à l'embouchure de la Loire d'un " emporium, immense marché où les Phéniciens, les Samnites et les Vénètes se rencontrèrent et se tendirent les mains". Cette question de Corbilo (ou Corbilon), le mythique port de l'étain, est alors la grande préoccupation des membres de la Société et fera l'objet d'une publication de son président, Édouard Sioc'han de Kersabiec en $1868^{91}$. Dans une note manuscrite en fin de volume, Parenteau donne la liste de toutes les personnalités à qui il a envoyé un exemplaire de sa publication ainsi que les réactions d'un certain nombre d'entre elles, généralement des membres de la Société archéologique ${ }^{92}$. Il n'apprécie guère les critiques et se pense incompris. À nouveau, en 1869, lors de la parution de son Catalogue $d u$ musée départemental d'archéologie, Parenteau supporte mal les réactions plutôt négatives à sa publication. La Société archéologique n'en fait même pas état dans ses comptes-rendus de séances. Ce deuxième catalogue du musée comporte 140 pages, 12 planches et des vignettes. Dans son introduction, Parenteau se plaint du manque de moyens qui ne lui a pas permis de faire graver plus d'illustrations :

" J'ai fait graver dix planches sur cuivre [...], et une douzaine sur bois [...]. Je ne comprends pas que l'on puisse, de nos jours, faire de l'archéologie sans l'aide du dessin et de la gravure [...]. Moins de texte et plus de planches, tout le monde y aurait gagné; mais, avec un budget de mille

90. Ibidem, p. 9 et 12 .

91. Sioc'HAN DE KERSABIEC, Édouard, “ Corbilon, Samnites, Vénètes, Namnètes, Bretons de la Loire ", BSANLI, 8, 1868, p. 53-88, 173-238 (avec carte), 281-306; BSANLI, 9, 1869, p. 17-59.

92. Parenteau, Fortuné, Le Fondeur du Jardin des Plantes de Nantes et son confrère de Rezé, 1868, musée Dobrée, fonds Parenteau, inv. 997.11.10 (1), p. 57-60. 
francs, il est difficile d'aller loin, et j'ai dû m'arrêter dans cette voie, bien à contrecœur, je l'avoue!»

Comme dans la première édition (1856), il suit un plan chronologique, pas toujours respecté, et une classification par matériau (monuments, céramique, armes et ustensiles). Le catalogue est d'abord présenté au baron Auguste Théodore de Girardot lors de la séance de la Commission administrative du musée de 1869 et il y reçoit un accueil élogieux ${ }^{93}$. Très fier, le conservateur estime que :

" Le jour où les quatre-vingt musées de province auront fait comme le musée de Nantes, et qu'il nous sera donné de pouvoir réunir sur le même rayon de la Bibliothèque tous les catalogues illustrés de nos Musées de France pour la somme de quatre ou cinq cents francs, ce jour-là, nous pourrons apprécier toutes nos richesses, et nous avons la conviction que la science archéologique aura fait un grand pas. "

À cette même séance, il est décidé que dix exemplaires du catalogue, "sans planches, solidement reliés, seront remis au concierge pour être loués aux visiteurs du musée les jours d'ouverture à raison de dix centimes l'un, tout le monde n'ayant pas six francs dans sa poche pour en faire l'acquisition ". En fait, cette publication a été jugée trop luxueuse dans la communauté scientifique parisienne et Parenteau accepte mal cette critique, somme toute relevant d'avantage de la jalousie. En effet, en 1881, dans l'introduction du Catalogue complet de ma collection archéologique ${ }^{94}$, il commente brièvement les réactions :

" J'acceptai de publier en 1869 une seconde édition sur beau papier de Hollande, et, plus tard, en séance générale à la Sorbonne, on déclara être trop beau pour un catalogue de musée de province, etc. Passons..."

En 1883, dans la biographie de son prédécesseur, Pitre de Lisle du Dreneuc, nous en apprend d'avantage ${ }^{95}$ :

" Le catalogue de 1869 a été édité avec luxe [...]. Un juge très sévère et très compétent a dit à ce sujet, dans un rapport à la Sorbonne : "M. Parenteau, le savant numismatiste, qui est le conservateur, à la fois honoraire et effectif, du Musée de Nantes, a eu le courage de s'attaquer à cette œuvre ingrate, mais méritoire, qu'on nomme un catalogue. Il a donné deux éditions de celui de Nantes : il n'y a qu'un reproche sérieux à faire à la seconde; car je ne parle pas des légères inexactitudes, inévitables dans des œuvres de cet ordre, que l'on a pu y signaler. Ce reproche, c'est que ce livre si utile est trop magnifique, qu'il a de trop belles planches, et que, partant, il n'est pas à la portée des visiteurs." Comme on le voit, la plainte du rapporteur frise de bien près l'élogieuse approbation. "

93. Note manuscrite de Fortuné Parenteau : Compte-rendu de la séance de la Commission administrative du musée de l'Oratoire de 1869, musée Dobrée, fonds Parenteau, Notes diverses, inv. 997.11.19, p. 83-87.

94. Parenteau, Fortuné, Catalogue complet de ma collection archéologique, $1^{\mathrm{er}}$ janvier 1881, manuscrit, musée Dobrée, fonds Parenteau, inv. 997.11.5, p. 3-4.

95. LISLE DU DRENEUC, Pitre de, « M. Fortuné Parenteau, conservateur du musée de l'Oratoire à Nantes ", $R B V, 3,1883$, p. 139-140. 
Même s'il est blessé par cette critique, Parenteau envisage immédiatement une troisième édition du catalogue :

"En prévision d'une nouvelle édition du catalogue du musée archéologique de Nantes ( $3^{e}$ édition), j'ai cru devoir faire relier ce volume [un exemplaire du catalogue de 1869] avec des cartons de manière à pouvoir classer et numéroter les nouvelles entrées du musée depuis 1869 jusqu'en $1879^{96}$. "

\section{Les dernières années de Parenteau et le don de sa collection au musée (1869-1882)}

Fortuné Parenteau poursuit très méthodiquement et consciencieusement son activité de "laborieux conservateur du musée ${ }^{97}$ ", et même s'il parvient à obtenir beaucoup de dons pour le musée, il doit souvent prendre sur ses revenus propres l'argent nécessaire à certains achats, assez rarement remboursés sur le budget du musée. Il polémique avec certains membres de la Société, avec Édouard de Kersabiec, notamment, au sujet des chenets à tête de bélier, dans lesquels Kersabiec croit voir des ornements de proue de navires égyptiens et phéniciens ${ }^{98}$, ou encore avec l'abbé Abel Cahour au sujet de la découverte d'une " colonne mérovingienne ${ }^{99}$ " dans les déblais de l'église Saint-Donatien à Nantes et que Cahour pense devoir provenir du tombeau des "Enfants nantais ». En 1869, Parenteau accueille le produit des fouilles de l'évêché ${ }^{100}$. Même s'il ne participe pas à la fouille, il dessine, en même temps que l'architecte diocésain Eugène Boismen ${ }^{101}$, un des bassins de l'église Saint-Jean du Baptistère, découvert en août 1868 par l'abbé Abel Cahour, président de la Société archéologique depuis décembre 1868, et il en fait faire un moulage en plâtre (fig. 19, et cahier couleur, fig. 20). En 1870, Parenteau participe aux fouilles du tumulus de l'âge du Fer de Barbechat (La Chapelle-Basse-Mer, Loire-Atlantique) entreprises le 19 avril 1870 avec Anatole de Barthélemy, membre de la Commission de la Carte des Gaules ${ }^{102}$.

96. PARENTEAU, Fortuné, note manuscrite sur un exemplaire du Catalogue de 1869, musée Dobrée, fonds Parenteau, inv. 997.11.8, p. 5.

97. BSANLI, 9, 1869, p. 14, 10 mars 1869.

98. BSANLI, 8, 1868, p. 259 et 260, 15 et 29 décembre 1868.

99. BSANLI, 12, 1873, p. 13, 4 février 1873.

100. "Débris de toutes sortes, marbres, granit, briques, fragments de ciment, bronze fondu, dents de sanglier, etc. ": BSANLI, 9, 1869, p. 88.

101. Après Viollet-le-Duc et Simon, et avant Chenantais et les frères Le Diberder, Boismen est l'un des architectes successivement utilisés comme praticiens par Thomas Dobrée pour la construction de son "palais".

102. Curieusement Fortuné Parenteau ne publie pas ce tumulus dont on ne connaît qu'une courte description : BSANLI, 10, 1870, p. 76, puis en 1872, BSANLI, 11, 1872, p. 37-39, Parenteau indique que la fouille "n'a pas donné tout ce qu'on en espérait [...], et que ce n'était qu'un amas de sépultures successives "; toutefois, il fait réaliser une maquette en liège du monument, destinée au musée de Saint-Germain-en-Laye. Quelques notes manuscrites de Parenteau sur la préparation et les débuts de la fouille dans un cahier Musée archéologique, non daté, nous apprennent que les subventions pour cette fouilles ont été importantes (archives du musée Dobrée). 
Figure 19 -

"Baptistère du $\mathrm{vI}^{\mathrm{e}}$ siècle. Cour de l'Évêché à Nantes août 1868 " bassin C de l'église SaintJean du Baptistère à Nantes

(Fortuné Parenteau, musée Dobrée, fonds Parenteau, inv. 997.11.10, p. 49 cl. musée Dobrée)

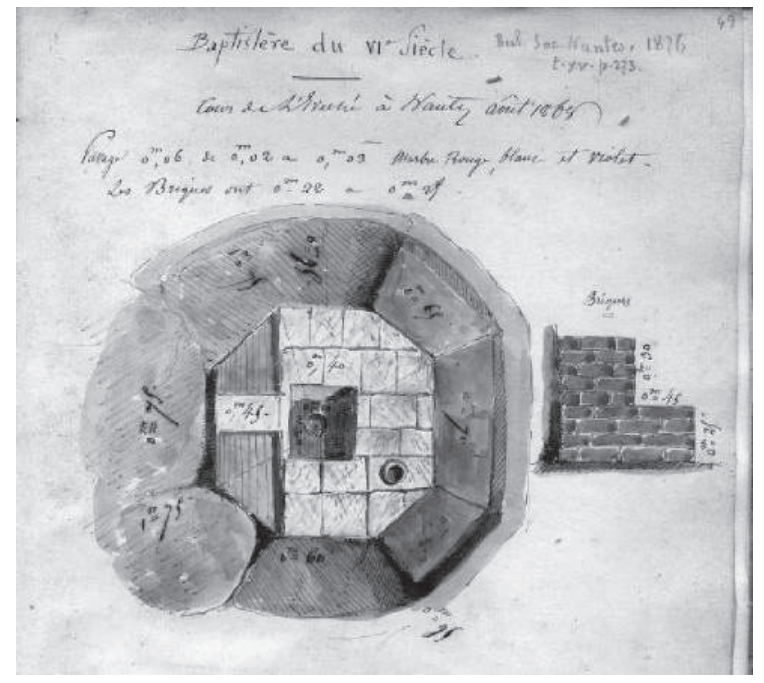

Figure 20 - Murus gallicus et fiches en fer de La Ségourie (Le FiefSauvin, M.-et-L.) ou de Vue (L.-A.) (dessin F. Parenteau dans MARTIN, Tristan et LEBEUF, Charles, Segora [Statio], Notes de F. Parenteau, Nantes, 1872, musée Dobrée, fonds Parenteau, inv. 997.11.21, p. 35, pl. III - cl. musée Dobrée)

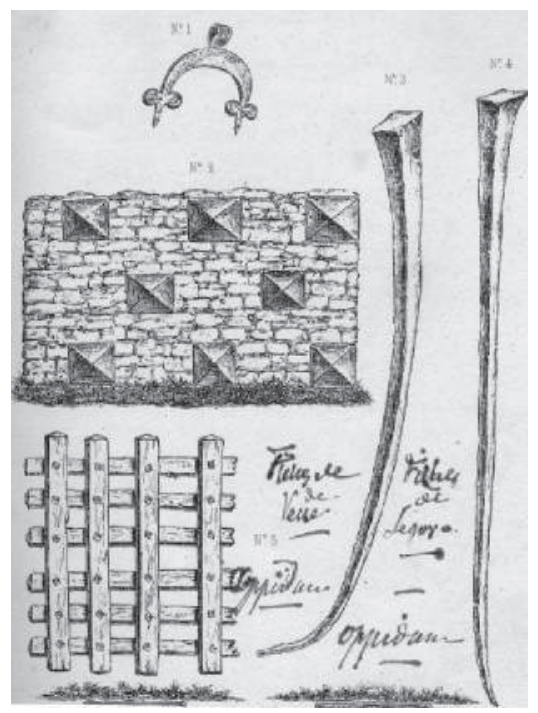

Il travaille également sur le site et le matériel des fortifications gauloises de la Ségourie (Maine-et-Loire) qu'il publie cette même année ${ }^{103}$ (fig. 20).

103. Parenteau, Fortuné, "Segora (statio)", BSANLI, 10, 1870, p. 81-91 (avec 4 pl.), musée Dobrée, fonds Parenteau, inv. 997.11.21. La planche concernant l'oppidum de La Ségourie montre deux fiches en fer du murus gallicus, l'une de La Ségourie et l'autre de Vue, ce qui peut paraître étrange car, dans une note manuscrite (inv. 997.11.20, p. 6), F. Parenteau nous apprend que les fiches en fer de l'oppidum de Vue ne lui sont adressées que le 23 janvier 1879 par le maire de Vue, M. Jacquelin. 
Déjà Fortuné Parenteau s'est lancé dans la préparation d'une nouvelle exposition, avec catalogue, qui lui doit sans doute beaucoup : l'exposition des beaux-arts, archéologie et peinture ancienne, organisée au Muséum d'histoire naturelle, encore installé par la Ville de Nantes à l'école de Chirurgie de la rue Saint-Léonard, en $1872^{104}$. Parenteau rédige la majeure partie du catalogue, consacrée à l'archéologie : les objets exposés font l'objet d'une brève notice et sont répartis par périodes et "séries" ou types d'objets (armes, céramiques, etc.), comme dans le Catalogue $d u$ musée de 1869, mais Parenteau y introduit des synthèses qui tournent parfois à la polémique, notamment, avec Benjamin Fillon au sujet des céramiques estampées tardives ou, encore, avec le baron Héracle Olivier de Wismes, sur les vases paléochrétiens trouvés près de la cathédrale de Nantes ${ }^{105}$. Les grands préteurs sont toujours les membres de la Société ou de l'élite intellectuelle nantaise, comme le baron Auguste de Girardot, le baron Louis des Dorides, Alexandre Perthuis, Charles Marionneau, Benjamin Fillon, Jean-Prosper Hyrvoix, Charles Seidler, le marquis de La Bretesche, le docteur Foulon, le baron Héracle Olivier de Wismes et René Huette. Les armes de l'âge du Bronze, les céramiques gallo-romaines et les bijoux mérovingiens y sont particulièrement bien représentés et montrent l'importance des collections locales en ce dernier tiers du XIX ${ }^{\mathrm{e}}$ siècle. Nombre de pièces n'entreront jamais au musée, ce que souligne d'ailleurs Pitre de Lisle du Dreneuc : "C'est un précieux recueil où nous trouvons enregistrés bien des trésors, dispersés maintenant dans des collections particulières, et réunis, à ce moment, dans les salles du Muséum de Nantes ${ }^{106}$." Après l'exposition, seules quelques pièces intégreront les collections du musée départemental d'Archéologie.

Parallèlement, Fortuné Parenteau poursuit ses collections et ses recherches sur les monnaies gauloises et publie, en 1873, l'amusante Odyssée de la bécasse en Gaule ${ }^{107}$ qui lui valut quelques remarques - bienveillantes quant au titre - de la part des numismates. Il encourage, par le vote d'une subvention de la Société archéologique, les fouilles du tumulus de Dissignac, appelé la Bosse de la Prière, récemment découvert par le lieutenant de vaisseau Arthur Martin qui en assumera la fouille avec l'ingénieur René de Kerviler ${ }^{108}$. Si Fortuné Parenteau ne participe pas, non plus, aux grandes fouilles de Saint-Donatien à Nantes, menées par l'abbé Cahour, qui se désigne lui-même comme " humble archéologue ${ }^{109}$ ", assisté de René

104. Catalogue raisonné de l'exposition des Beaux-Arts, archéologie et peinture ancienne, Nantes, Jules Grinsard, 1872.

105. Ibidem, p. 71-73.

106. Lisle du Dreneuc, Pitre de, «M. Fortuné Parenteau, conservateur du musée de l'Oratoire de Nantes ", $R B V, 1883$, p. 140.

107. BSANLI, 12, 1873, p. 25-28 (1 pl.).

108. BSANLI, 12, 1873, p. 109-110, 19 août 1873, et p. 122-124, 2 décembre 1873; MARTIN, Arthur et PocARD DE KERVILER, René, "Fouilles du tumulus de Signac en Saint-Nazaire ", BSANLI, 12, 1873, p. 172-192 (6 pl.).

109. BSANLI, 12, 1873, p. 125-126, 23 décembre 1873; "Fouilles faites en 1873 à SaintDonatien ", BSANLI, 13, 1874, p. 129. 
de Kerviler, de Louis Petit et de Paul Anizon, il est toutefois appelé comme expert pour donner soin avis sur un certain nombre d'objets, notamment sur des céramiques de l'Antiquité tardive et de l'époque mérovingiennes. Il est intéressant de constater qu'à l'occasion de cette fouille, l'archéologie locale prend une nouvelle forme, plus scientifique et pluridisciplinaire, puisque le groupe des " archéologues " est constitué d'un responsable du chantier, l'abbé Abel Cahour, d'un archéologue-dessinateur, Louis Petit, qui décrit les trouvailles, d'un ingénieur des Ponts-et-Chaussées, René de Kerviler, qui porte un regard critique sur la méthode de fouilles, examine les couches archéologiques et trace les plans, et d'un médecin, le docteur Paul Anizon, qui examine le contenu de deux des sarcophages découverts et d'une urne funéraire.

Dans ces années soixante-dix, le regain d'intérêt pour les monuments mégalithiques est évident; il est porté par le révérend William Collings Lukis $^{110}$, par Arthur Martin ${ }^{111}$, René de Kerviler et Héracle Olivier de Wismes ${ }^{112}$ et donne lieu à nombre de discussions au sein de la Société sur les pierres gravées ou les pierres à cupules. Toujours préoccupé par l'enrichissement du musée dont il a la charge, Parenteau est parfois maladroit, comme, par exemple, à propos du mobilier des tumulus de Pornic, fouilles de la Société archéologique confiées au baron de Wismes : Parenteau s'empare précipitamment des objets fouillés qu'il fait mettre en sécurité dans les vitrines du musée et se fait rappeler à l'ordre par la Société archéologique :

" M. Parenteau, conservateur du musée, répond qu'il a cru devoir s'emparer des objets avant la remise officielle, pour mieux les protéger contre toute lésion [...]. M. le président [Charles Marionneau], dit que la Société archéologique était seule propriétaire des objets trouvés dans le tumulus de Pornic, qu'elle consent à en faire don au musée départemental, suivant sa promesse, et demande à M. le conservateur d'ouvrir les vitrines à la fin de la séance, pour satisfaire le légitime désir des assistants ${ }^{113}$. "

Parenteau parvient aussi à faire acheter par le musée l'importante collection de haches polies du Vendéen Léon Ballereau ${ }^{114}$.

Profitant d'une série de communications au sein de la Société archéologique sur les fouilles du lac de Paladru (Isère) et de la perspective du Congrès de la Société française pour l'avancement des sciences, en août 1875, Parenteau cherche à réaliser son vieux rêve : fouiller le lac de Grand-Lieu ${ }^{115}$. Ce projet ne verra jamais le jour car " tout était prêt, et les fouilles allaient com-

110. LuKIS, William Collings, "Sur la dénomination des dolmens ou cromlechs ", BSANLI, 9, 1869, p. 175-184 (2 pl.), précédé d'une note de Fortuné Parenteau encourageant la Société à publier cet article. Id., "Sur la série des monuments en pierre brute ", BSANLI, 14, 1875, p. 181-220.

111. Tumulus de Dissignac, pierres gravées de Meniscoul, BSANLI, 1873 et 1874.

112. WismEs, Olivier de, "Le tumulus des Trois squelettes à Pornic (Loire-Inférieure) ", BSANLI, 15, 1876, p. 199-271 (7 pl.).

113. BSANLI, 14, 1875, p. 179, 6 décembre 1875.

114. BSANLI, 14, 1875 , p. 84 , 4 mai 1875 ; le prix est de $400 \mathrm{~F}$.

115. BSANLI, 14, 1875, p. 22, 2 février 1875. 
mencer, lorsqu'arrivèrent l'invasion prussienne et nos désastres; tout fut enrayé, et les fonds avancés furent rendus! Espérons qu'avec les beaux jours, il nous sera donné de pouvoir reprendre nos pacifiques recherches ${ }^{116}$ ". À l'occasion de la tenue de ce congrès, une exposition sur "l'archéologie préhistorique, gauloise, etc. " est organisée par la Ville et la Société académique de Nantes, du 19 au 26 août 1875, au foyer du théâtre de la Renaissance ${ }^{117}$. Contrairement à l'exposition de 1872, les objets proviennent de tout l'Ouest, de la Bretagne à l'Aquitaine, et on a pu y voir, notamment, les très belles pièces en variscite du tumulus Saint-Michel de Carnac, fouillé en 1862 par René Galles et conservées au musée de la Société Polymathique de Vannes, ou encore les collections préhistoriques fouillées au Mont-Dol (Ille-et-Vilaine) par M. Sirodot, doyen de la Faculté des Sciences de Rennes. Le musée préhistorique de Bordeaux prête également de "beaux objets des âges de Pierre et de Bronze ". Il semble aussi que ce soit la première fois que le coffret funéraire du cœur d'Anne de Bretagne soit exposé au public, "posé sur lit de velours rouge " avec la gravure du "reliquaire " par Octave de Rochebrune ${ }^{118}$. Comme toujours, les collectionneurs régionaux prêtent leurs pièces les plus importantes (Benjamin Fillon, Fortuné Parenteau, Charles Seidler, le baron Auguste Théodore de Girardot, l'abbé Baudry) et des vitrines spécifiques leur sont consacrées. Trois vitrines présentent l'archéologie locale : la première porte sur l'âge de la Pierre et l'âge du Bronze, la seconde est consacrée aux collections de Fortuné Parenteau et la troisième aux collections du musée départemental d'Archéologie. Enfin, d'autres vitrines sont consacrées aux collections extra européennes (armes gabonaises et polynésiennes, notamment). Une fois encore, la Ville fait appel à Fortuné Parenteau pour la rédaction du catalogue.

Curieusement, mais sans doute parce qu'il travaille à son propre Inventaire archéologique, Fortuné Parenteau ne suit pas les importantes découvertes de René de Kerviler lors du creusement du bassin de Penhoët commencé en $1874^{119}$. Au sujet de cette fouille et de la théorie du "Chronomètre préhistorique ", le conservateur se contente de noter dans ses documents personnels une réflexion du directeur du musée de SaintGermain-en-Laye, Louis Laurent Gabriel de Mortillet, qu'il qualifie d'ailleurs d'insolent : "Dans le dépôt limoneux de la baie de Penhouët, près de SaintNazaire (Loire-Inférieure). Récoltes Kerviler [...]. Le saint homme voulait saper toute la chronologie préhistorique ${ }^{120}$. »Pendant presque toute l'année

116. Parenteau, Fortuné, « Fouilles du lac de Grand-Lieu; les lacs de Paladru et de GrandLieu ", BSANLI, 14, 1875, p. 147-150.

117. Compte-rendu des objets exposés au foyer du théâtre de la Renaissance, Nantes, Charpentier imp., 1875.

118. Ibidem, p. 4.

119. KERVILER, René de, « L'Âge du Bronze et les Gallo-Romains à Saint-Nazaire-sur-Loire ", BSANLI, 15, 1876, p. 287-316 (9 pl.). SANTROT, Jacques, « Le chronomètre préhistorique de Saint-Nazaire ", dans Place Publique, 2007, 2, p. 64-65.

120. PARENTEAU, Fortuné, document préparatoire au Catalogue complet de ma collection archéologique, de 1881, musée Dobrée, fonds Parenteau, inv. 997.11.5, p. 290. Gabriel de Mortillet a publié dans les Bulletins et mémoires de la Société d'Anthropologie de Paris, 1878 , un article intitulé " Le chronomètre du bassin de Penhouët réduit à sa simple 
1876, Parenteau ne participe à aucune des séances de la Société archéologique et n'y revient qu'en 1877, à l'occasion des importantes découvertes gallo-romaines, soutenues financièrement par le musée, faits dans les fondations du rempart gallo-romain de Nantes, rue de Strasbourg et rue GardeDieu (fig. 21 à 23 et cahier couleur, fig. 21, 22).

\section{Figure 21 - Fouilles de la rue de Strasbourg à Nantes \\ (dessin de Gustave Bourgerel, 8 février 1877, musée Dobrée, inv. $56.5244-$ cl. musée Dobrée)}
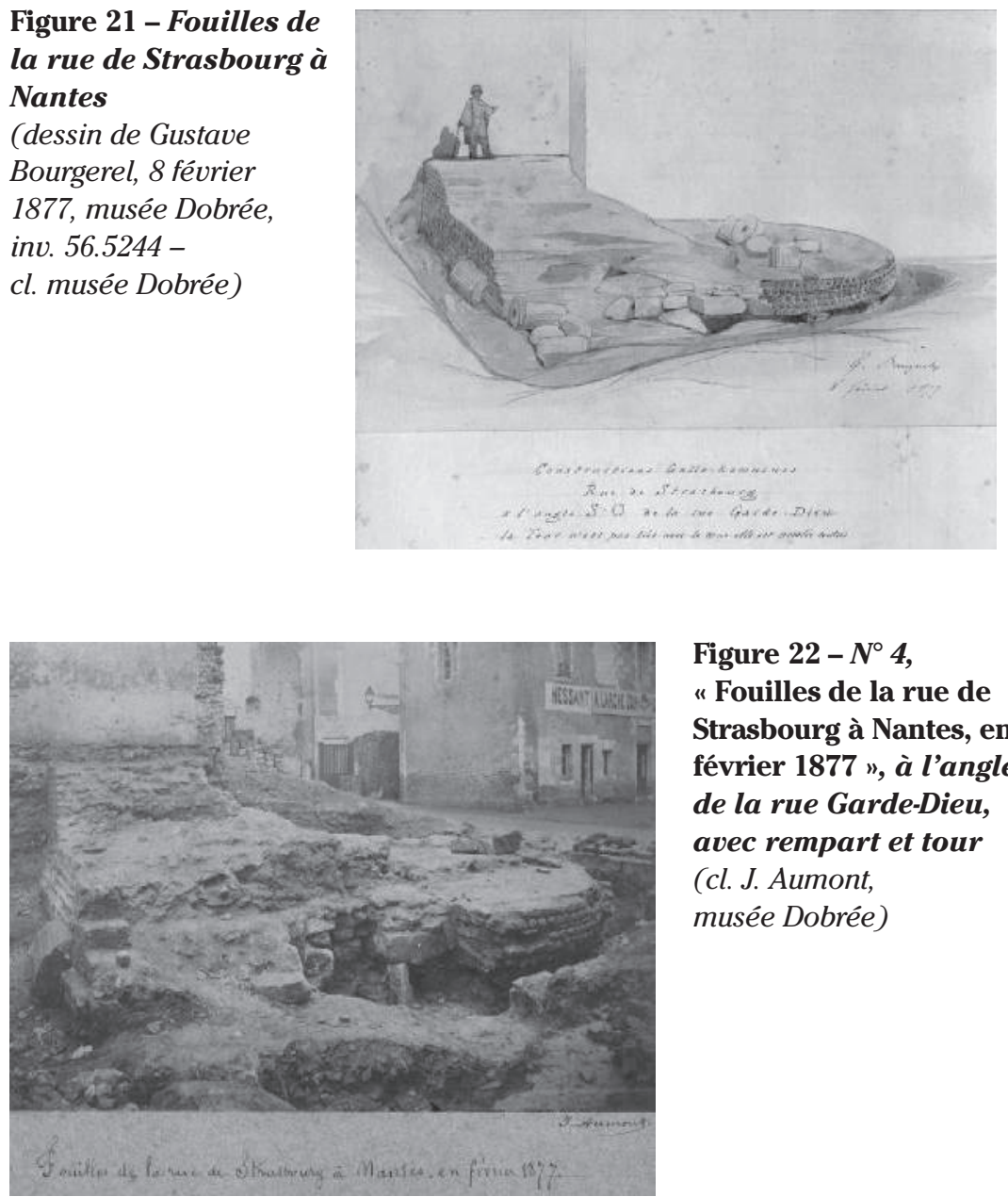

Figure $22-N^{\circ} 4$,

"Fouilles de la rue de Strasbourg à Nantes, en février 1877 ", à l'angle de la rue Garde-Dieu, avec rempart et tour (cl. J. Aumont, musée Dobrée)

valeur " dans lequel il réfute la thèse de René de Kerviler; ce texte sera lu lors de la séance du 19 février 1878 de la Société archéologique : BSANLI, 17, 1878, p. 12. 
Figure 23 - " Temple d'Apollon (Nantes), recueilli au musée par M. Gibouin ", de F. Parenteau (chapiteau corinthien) (chapiteau corinthien de la rue de Strasbourg, et colonne cannelée de Saint-Donatien, dans le musée de l'Oratoire, cl. J. Aumont, musée Dobrée, fonds Parenteau, inv. 997.11.28 cl. musée Dobrée)

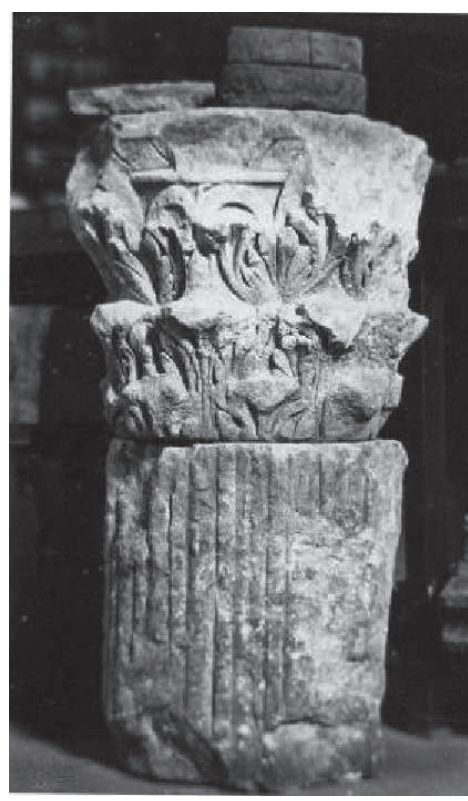

Persuadé que l'on y a découvert les restes d'un temple d'Apollon ${ }^{121}$, Parenteau prépare une publication à ce sujet et rédige une communication pour la Société archéologique, lue à la séance du 9 janvier 1877 et qui est accueillie avec certaines critiques : l'abbé Cahour et le baron de Wismes estiment que le propos n'est pas suffisamment étayé et que les deux sphinges découvertes en 1849 et 1876 et placées par Parenteau de part et d'autre du fronton du temple, n'ont jamais été un symbole d'Apollon ${ }^{122}$. Il ne publiera pas le texte qu'il a préparé avec le dessin reconstitutif du temple présumé (fig. 24) et n'assistera pas, pendant un certain temps, aux séances de la Société archéologique.

Le conservateur est sans doute également préoccupé par la réorganisation de la Commission administrative du musée, décidée le 4 août 1876 en présence du préfet de la Loire-Inférieure, le vicomte Camille Malher, et du maire de Nantes, Charles Lechat. Il semble que ce soit à la demande de Fortuné Parenteau que cette décision soit prise, la Commission administrative mise en place en 1861 ne s'étant jamais réunie. Le préfet prend un arrêté pour la reconstitution de la Commission $^{123}$ : le remplacement des membres décédés ou démissionnaires comme le baron de Girardot, président de la Commission, et la nomination de

nouveaux membres " afin qu'ainsi composée, cette Commission prit tout initiative pour maintenir au rang qu'il doit occuper, parmi les musées départementaux, celui de la Loire-Inférieure ${ }^{124}$ ". Les dix-sept membres de la nouvelle Commission administrative sont désormais le préfet de la Loire-Inférieure, qui la préside, le secrétaire général de la Loire-Inférieure, qui en est le vice-

121. Parenteau, Fortuné, Temple d'Apollon à Nantes sous l'empereur Auguste, 1876 , manuscrit, musée Dobrée, fonds Parenteau, inv. 997.11.16.

122. BSANLI, 16, 1877, p. 6-7, et manuscrit Parenteau, musée Dobrée, fonds Parenteau, inv. 997.11.16, p. 185-191.

123. Archives du musée Dobrée, arrêté du 2 août 1876.

124. Compte rendu de la réunion de la Commission administrative du musée départemental d'Archéologie du 4 août 1876, archives musée Dobrée. 
Figure 24 - « Temple d'Apollon à Nantes sous l'empereur Auguste - restitution"

(Fortuné Parenteau, musée Dobrée, fonds Parenteau, Nantes, 1876, inv. 997.11.16, p. $183, n^{\circ} 3-$ cl. musée Dobrée)

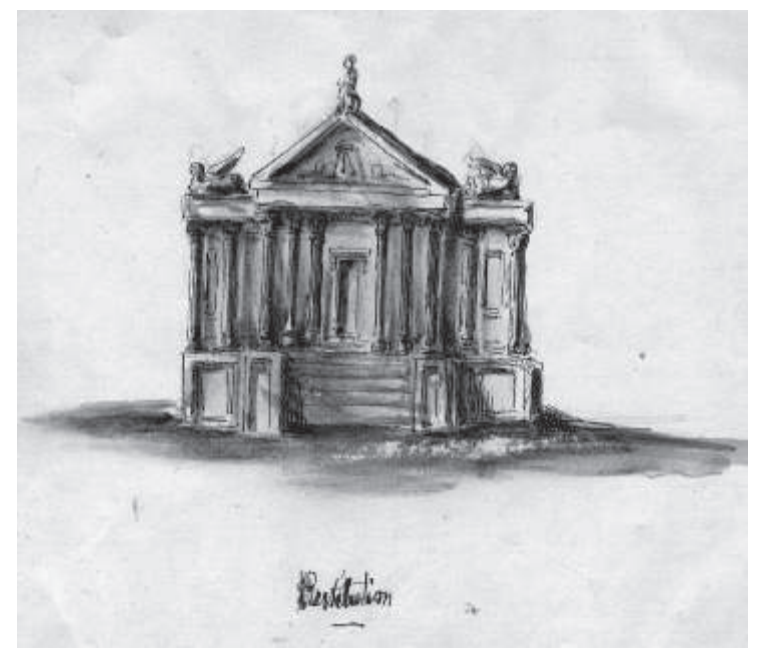

président, Fortuné Parenteau, conservateur, Mgr Félix Fournier, évêque de Nantes, Charles Lechat, maire de Nantes, Gustave Bourgerel, architecte du Département, Charles Marionneau, président de la Société archéologique, Henri de Cornulier, conseiller général, Joseph Rousse, conseiller général, le baron Héracle Olivier de Wismes, artiste et littérateur, Thomas Dobrée, $\operatorname{archéologue}^{125}$, Stéphane Halgan, sénateur et numismate, Paul Eudel, armateur, négociant, collectionneur et chroniqueur d'art, Paul Nau, architecte, Alexandre Perthuis, numismate et trésorier du musée, le vicomte Édouard Sioc'han de Kersabiec, conseiller de la Préfecture, le baron Auguste de Girardot, ancien secrétaire général de la Préfecture. Dans son article II, l'arrêté mentionne également que la Commission se réunira tous les mois dans le local du musée sous la présidence du préfet. À la suite de l'exposé de l'état des finances du musée par Alexandre Perthuis, son trésorier, le conservateur fait observer que "bien que l'allocation accordée par le Département soit minime, il reste encore un boni [...] et que si ce résultat est d'une bonne administration, il n'en est pas moins vrai qu'il n'est obtenu qu'en restreignant les acquisitions d'objets qu'il serait si désirable parfois d'introduire au Musée pour en augmenter les intéressantes collections ". En rappelant que le conservateur du musée et la Société archéologique ont rendus de grands services à la Ville par l'organisation des expositions de 1872 et 1875, le maire de Nantes, Charles Lechat, propose une augmentation de sa subvention pour "venir en aide au développement d'une science dont la culture fait honneur au Département ". À cette même séance, le conservateur demande la reconstitution de la Commission de surveillance du musée départemental d'Ar-

125. Thomas Dobrée sera également qualifié de "numismatiste ". Archives du musée Dobrée, document de préparation au Règlement du musée départemental d'Archéologie de 1894, non daté (1888?). 
chéologie, qui lui est accordée ${ }^{126}$. C'est à la réunion de l'année suivante, en mars 1877, que les fonctions de la Commission sont fixées par son président, M. de La Giraudais, vice-président du Conseil de la Préfecture, en l'absence du préfet ${ }^{127}$. La première mission est de "venir en aide à $M$. le conservateur soit par des conseils scientifiques ayant trait aux acquisitions et à la bonne disposition du musée "; la seconde est "d'émettre des vœux pris de l'autorité compétente pour l'amélioration du musée " et la troisième, "de vérifier les comptes financiers de la gestion". Le conseiller général Henri de Cornulier propose également la mise en place d'un règlement pour déterminer les rôles et les modes d'actions respectifs. À la demande du baron de Wismes, une sous-commission composée de Charles Marionneau, Paul Eudel et Héracle Olivier de Wismes est chargée de rédiger le règlement qui doit se faire en accord avec le conservateur et être approuvé par le préfet. Il faudra attendre douze ans pour qu'il soit enfin achevé ${ }^{128}$. Le conservateur dresse également à cette occasion un état assez catastrophique du musée de l'Oratoire :

"Mais ces richesses [du musée], comment sont-elles placées? Où sontelles exposées? Les unes le sont très insuffisamment; d'autres ne le sont pas ou le sont indignement. Elles ont reçu une hospitalité provisoire dans des vitrines qui les contiennent sans les montrer. Le contenant est absolument indigne du contenu et, de fait, les curieux et les hommes de sciences (et la curiosité appelle et engendre la science) ne peuvent ni les apprécier ni les étudier. Un musée ne doit pas être un entassement, mais un véritable étalage d'objets scientifiques."

Des plans d'implantation de vitrines sont fournis par l'architecte départemental Gustave Bourgerel, avec un devis ${ }^{129}$. Le projet est renvoyé par le Conseil général à une cession départementale ultérieure, mais transmis sans attendre au préfet. Seuls 2000 F seront votés en 1878 auxquels la Ville de Nantes ajoutera $1000 \mathrm{~F}^{130}$. À l'occasion de cette séance, le conservateur transmet à la Commission son dernier ouvrage, Fragments d'architecture et de sculpture, qui n'a malheureusement pas été conservé. Il fait également part de la préparation de son Inventaire archéologique ${ }^{131}$ en informant les membres qu'il s'est permis de faire graver dans ses planches des objets appartenant au musée, ce qui va provoquer une longue et difficile discussion au sein de la Commission, car le baron Héracle Olivier de Wismes,

126. Archives du musée Dobrée, lettre du préfet Camille Malher du 2 août 1876.

127. Compte rendu de la réunion de la Commission du 26 mars 1877, archives du musée Dobrée.

128. Règlement du musée départemental d'Archéologie de la Loire-Inférieure, 6 février 1889, manuscrit, archives du musée Dobrée. Deux autres règlements seront établis en 1894 et 1910 .

129. Devis estimé à $10000 \mathrm{~F}$ dans le compte rendu de la réunion de la Commission du 26 mars 1877, archives du musée Dobrée.

130. Commission administrative du 11 mars 1878, archives du musée Dobrée.

131. PARENTEAU, Fortuné, Inventaire archéologique précédé d'une introduction à l'étude des bijoux, Nantes, Vincent Forest et Émile Grimaud, imprimeurs-éditeurs, 1878, 140 p., 62 pl. Pitre de Lisle du Dreneuc sera chargé par la Société archéologique de faire le compterendu de cet ouvrage : $B S A N L I, 18,1879$, p. 248-250. 
notamment, conteste à Parenteau le droit absolu de reproduction d'objets appartenant à des tiers et placés dans des collections publiques.

Enfin, la participation du musée à l'Exposition universelle de 1878 est le dernier grand sujet adopté par la Commission : "La sous-commission nommée pour la réinstallation du musée est chargée de déterminer les envois à faire et le mode de ces envois, et, enfin, de prendre les précautions pour que ces richesses scientifiques adressées à Paris en reviennent intactes. " Dans le même temps, un débat s'instaure au sein de la Société archéologique ${ }^{132}$. Le conservateur propose d'y envoyer "tout ce qu'il possède sur l'outillage des premiers ateliers de fer de notre contrée ". Lors d'un séjour à Paris en avril, Charles Marionneau, président de la Société, a pu constater que « rien n'était prêt. Les caisses étaient fermées, et il était bien inutile de rechercher au milieu de l'encombrement général les objets appartenant à notre musée. Au $1^{\text {er }}$ juin peut-être aurons-nous le plaisir de les savoir installés ${ }^{133}$ ". Les laissezpasser de Fortuné Parenteau et Pitre de Lisle du Dreneuc (fig. 25 à 29) nous permettent de savoir qu'ils y ont participés tous les deux, dans la classe des

Figures 25 et 26 - Carte d'exposant en sciences anthropologiques de Fortuné Parenteau à l'Exposition universelle de Paris, 1878, recto et verso (musée Dobrée, fonds Parenteau, inv. 58.554 - cl. musée Dobrée)
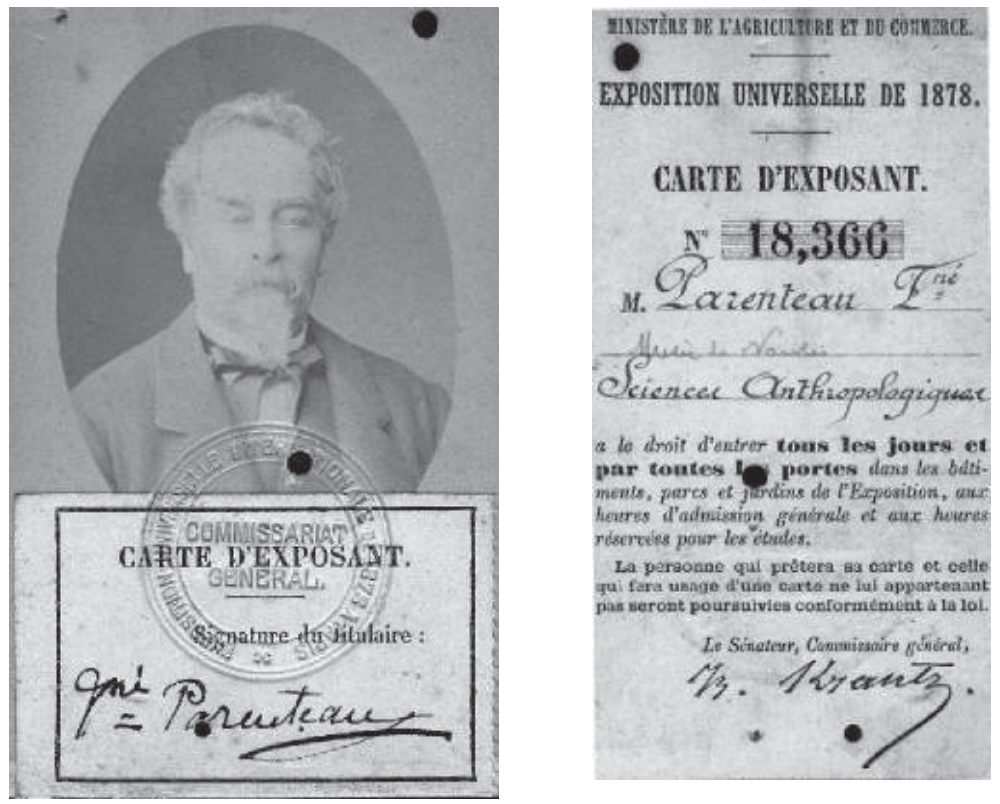

132. BSANLI, 16, 1877, p. 214, 6 novembre 1877.

133. BSANLI, 17, 1878 , p. 20,7 mai 1878 . 
Figures 27, 28, 29 - Carte d'exposant en sciences anthropologiques de Pitre de Lisle du Dreneuc à l'Exposition universelle de Paris, 1878, et médaille offerte pour services rendus à MM. Pitre et Georges de Lisle du Dreneuc

(coll. part., cl. musée Dobrée)
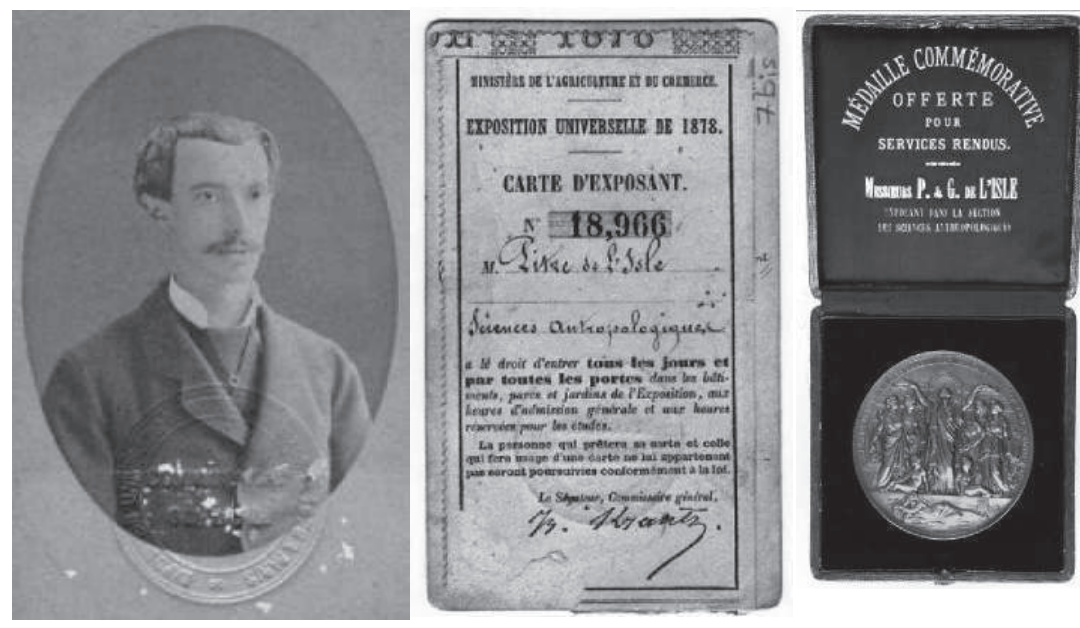

Sciences anthropologiques, le premier au titre du musée, le second, avec son frère Georges, sans doute, comme spécialistes des monuments mégalithiques. Un autre Nantais, d'origine anglaise, le collectionneur Charles Seidler, y participe également.

Le grand œuvre de Parenteau reste la rédaction de l'inventaire de ses collections. Comme il nous l'apprend dans le manuscrit du Catalogue complet de ma collection archéologique ${ }^{134}$ en 1881, Parenteau s'atèle à la rédaction de la publication de ses collections : son Inventaire archéologique et sa collection personnelle de bijoux. Dans l'introduction manuscrite de l'Inventaire de 1881 :

«Depuis cette époque [la publication du catalogue de 1869], j'ai pu faire marcher parallèlement sans le nuire et sans le heurter, la collection particulière du musée départemental, et la mienne, propre, plus tard, espérons qu'elles se réuniront, si rien ne vient s'y opposer. "

" J'ai consacré une partie de ma vie à réunir, dans le Poitou, mon pays natal, et dans la Bretagne, mon pays d'adoption, les bijoux et les bibelots qui forment ma collection, ainsi que les documents qui les expliquent. Arrivé à la maturité, j'ai cru devoir en faire l'inventaire. Chez les uns, l'inventaire précède la vente; pour moi, l'inventaire précédera peut-être de peu de temps

134. Parenteau, Fortuné, Catalogue complet de ma collection archéologique, $1^{\mathrm{er}}$ janvier 1881, manuscrit, musée Dobrée, fonds Parenteau, inv. 997.11.5, p. 4. 
l'immobilisation de ma collection dans un musée public et accessible à tous [...]. J'ai cru bien faire en joignant à cet inventaire la description des objets les plus curieux que, depuis dix ans, j'ai fait entrer au musée de l'Oratoire (que j'ai l'honneur de diriger depuis vingt ans), en 1869."

Le premier Inventaire archéologique de ses collections occupe Parenteau de 1865 à 1878, date de sa parution ${ }^{135}$. Il le dédit à sa femme et le fait précéder d'une citation qui lui est chère : "Chacun creuse le sol où chacun met son grain ", déjà employée dans ses publications. Le musée Dobrée conserve deux versions de cet Inventaire archéologique, correspondant à deux stades d'avancement du manuscrit. Le document préparatoire ${ }^{136}$, le plus complet, car enrichi de nombreuses planches d'objets comparatifs ou de courriers de chercheurs, est déjà précédé de "l'introduction à l'étude des bijoux " qui est un simple collage annoté de pages imprimées de différentes publications rédigées dès le $1^{\mathrm{er}}$ janvier $1858^{137}$, avec quelques variantes jusqu'en 1875 . Il comporte ensuite 61 planches gravées à l'eau-forte avec, en regard, la notice manuscrite de chaque objet ${ }^{138}$. Pour certaines, le dessin préparatoire, sur calque et à l'encre de Chine de la planche est conservé (fig. 30 et 31).

\section{Figures 30 et 31 - Calque préparatoire et planche gravée de l'Inventaire archéologique de Fortuné Parenteau, 1878}

(musée Dobrée, fonds Parenteau, inv. 997.11.4, p. 74 et 75 - cl. musée Dobrée).
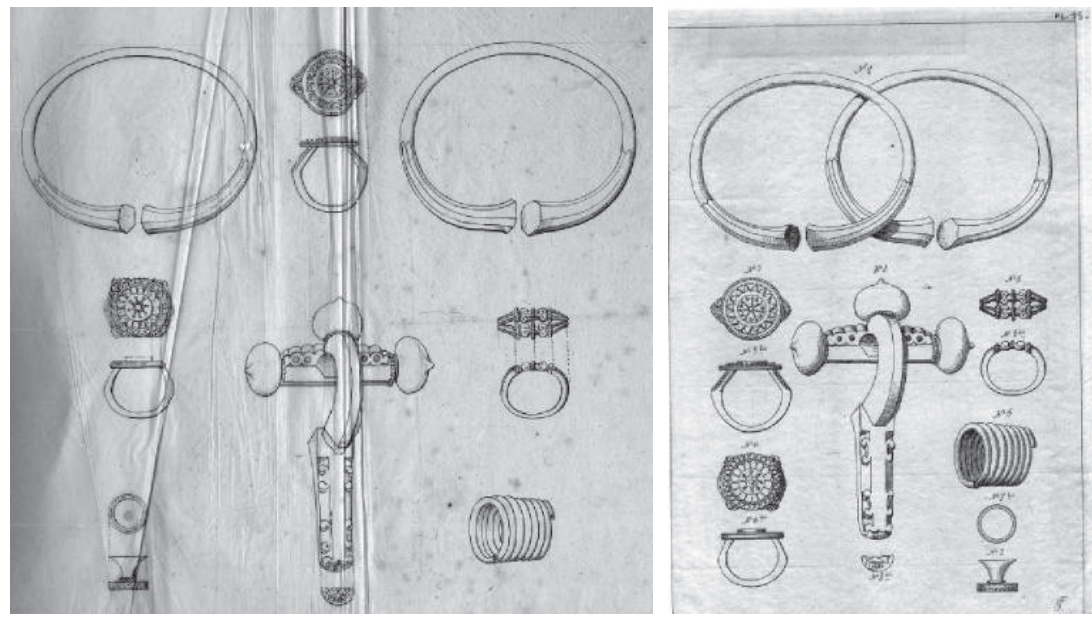

135. PARENTEAU, Fortuné, Inventaire archéologique précédé d'une introduction à l'étude des bijoux, Nantes, V. Forest et E. Grimaud, 1878.

136. PARENTEAU, Fortuné, Inventaire archéologique, manuscrit, musée Dobrée, fonds Parenteau, inv. 997.11.4.

137. PARENTEAU, Fortuné, Catalogue descriptif d'une collection de bijoux, 1858, manuscrit, musée Dobrée, fonds Parenteau, inv. 997.11.9, p. 149, 151 et 153.

138. Il fera supprimer la planche 60 dans l'édition imprimée. 
L'auteur a parfois ajouté, en regard de planches d'objets de sa collection, des planches de pièces appartenant à des musées ou à des collectionneurs privés (Fig. 32 à 34). Dans d'autres cas, des découpages de textes publiés concernant le même type d'objets ou encore des courriers d'échanges avec des chercheurs sont annexés entre les planches. Ce qui permet de comprendre la méthode de travail de Parenteau.

\section{Figure 32 - Légende manuscrite en regard de la planche 21, terres cuites du Bas-Empire, de l'Inventaire archéologique de Fortuné Parenteau, 1878}

(musée Dobrée, fonds Parenteau, inv. 997.11.4, p. 91 - cl. musée Dobrée)

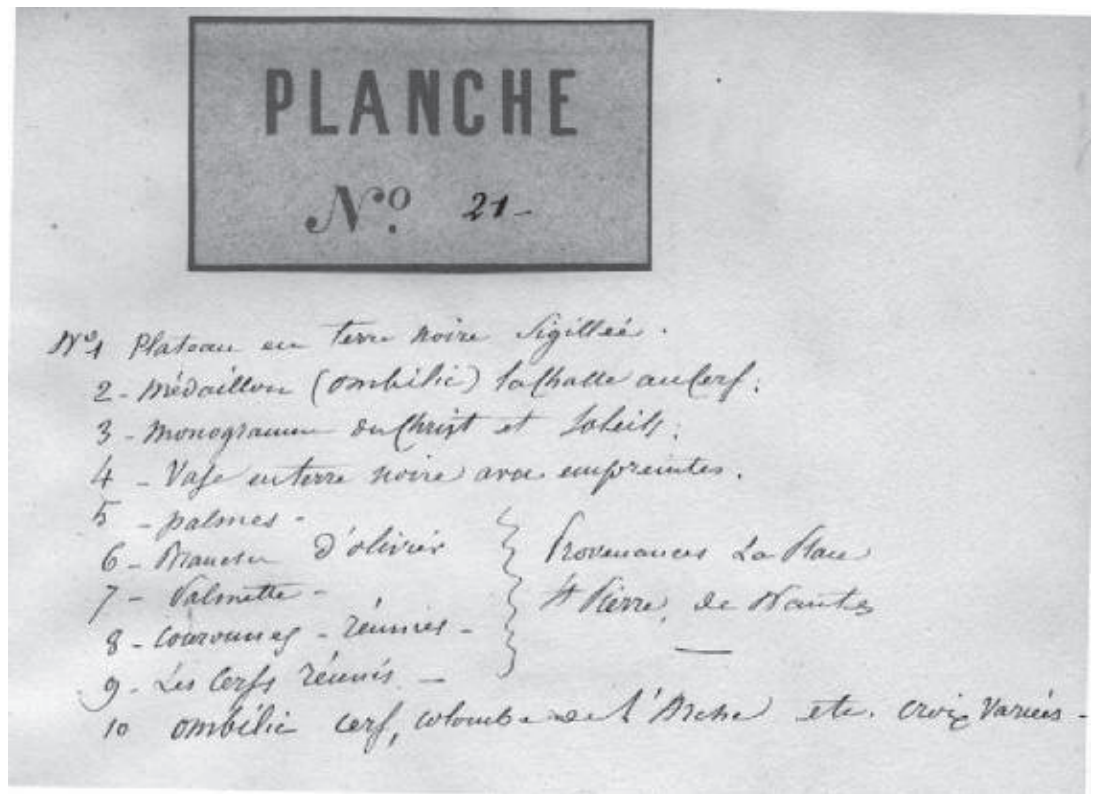

La seconde version ${ }^{139}$, définitive et totalement imprimée, est composée de 62 planches gravées, avec, en regard, les notices des objets et de courts textes de synthèse sans véritable ordre chronologique (âge de la pierre polie, céramique gauloise, céramique gallo-romaine, âge du Bronze, outillage primitif du Fer en Gaule, armes de fer, âge d'or et âge du cuivre, etc. ${ }^{140}$. En fait, cet Inventaire des collections archéologiques de Parenteau suit à peu près le même plan que la seconde édition du Catalogue du musée

139. PARENTEAU, Fortuné, Inventaire archéologique précédé d'une introduction à l'étude des bijoux, Nantes, 1878, musée Dobrée, fonds Parenteau, inv. 997.11.18.

140. Fortuné Parenteau se plaint : "Mon graveur ayant mis un peu de fantaisie dans le numérotage des objets gravés, je suis obligé de suivre l'ordre indiqué par lui ": PARENTEAU Fortuné, Inventaire archéologique précédé d'une introduction à l'étude des bijoux, Nantes, 1878, nota bene, p. 14. 
Figures 33 et 34 - Planche XXI, céramiques paléochrétiennes, dans l'Inventaire archéologique de Fortuné Parenteau, 1878, et, en regard dans le même ouvrage, Baron de Wismes (Exposition), Poteries mérovingiennes de la place Saint-Pierre de Nantes, pl. XI, planche d'objets paléochrétiens appartenant au baron de Wismes et lithographiés par J. Grinsard, Fortuné Parenteau, 1878

(musée Dobrée, fonds Parenteau, inv. 997.11.4, p. 88 et 89 - cl. musée Dobrée)
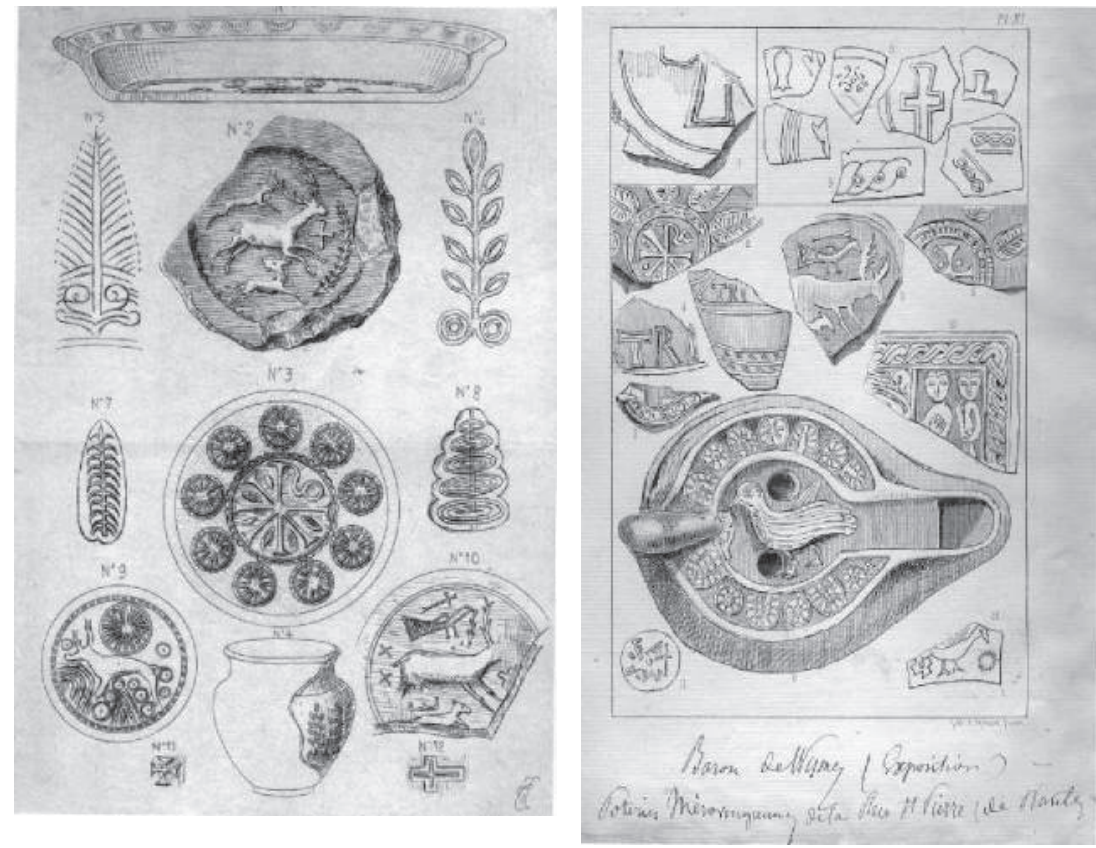

archéologique de 1869, avec un classement chronologique par matériau. Il y mêle donc, à titre de comparaison et pour les éclairer, des objets lui appartenant, dont beaucoup proviennent de fouilles locales et qu'il a conservé dans ses collections, des objets du musée départemental ou encore provenant de collections particulières, ce qui a soulevé une polémique dans la communauté intellectuelle locale (fig. 35 et 36). On connaît l'attachement de Parenteau à l'illustration des collections et, malgré le nombre de planches gravées à l'eau-forte, il exprime son regret "de ne pouvoir aller au-delà; mais, en conscience, il était temps de s'arrêter", et d'ajouter "mes planches, en ce qui regarde ma collection, en empêcheront l'évaporation ${ }^{141}$ ". Comme il l'exprime peu après, il s'agit d'une "publication de luxe que j'ai cru pouvoir me permettre ${ }^{142}$ ".

141. Dans l'introduction, p. 3.

142. Catalogue complet de ma collection archéologique, janvier 1881, manuscrit, musée Dobrée, fonds Parenteau, inv. 997.11.5, p. 4. 
Fig. 35 - Clef de l'abbatiale Saint-

Philbert-de-Grand-Lieu (L.-A.) et clefs antiques

(Fortuné Parenteau, musée Dobrée, fonds Parenteau, inv. 997.11.5, p. $74-$ cl. musée Dobrée)
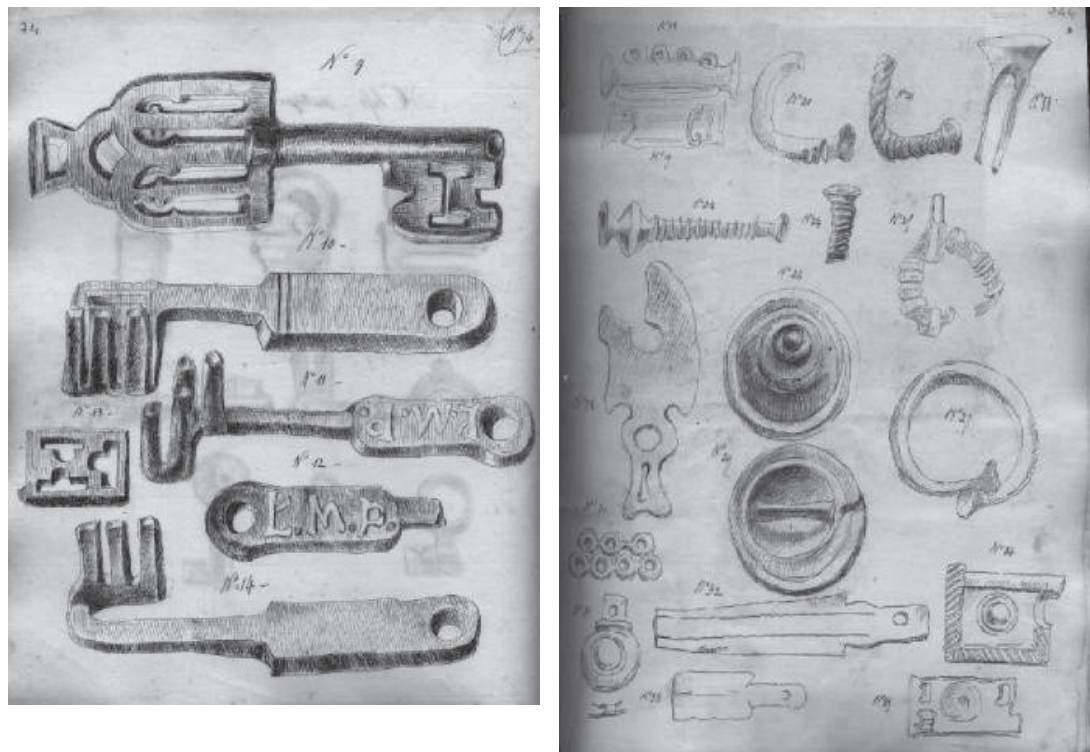

Quatre ans avant sa mort, Parenteau est déjà très fatigué car, à la fin de son Inventaire, il écrit : "Lors de la rédaction et de l'impression de mon Inventaire, garde-malade et très fatigué moi-même, je n'eus peut-être pas à ma disposition toute ma présence d'esprit et ma liberté d'action ${ }^{143}$. "Même s'il est prêteur, on ne sait pas s'il prend une part active à l'exposition parisienne du musée d'Ethnographie du Trocadéro (salle d'Anthropologie) de 1878. Par le baron de Wismes, et comme dans toutes les expositions déjà citées, on apprend que les collectionneurs nantais, membres de la Société, y ont participé ${ }^{144}$ : Charles Seidler, "dont l'exposition était une des plus belles", Parenteau, Marionneau et lui-même font partie de ces prêteurs ${ }^{145}$. Parenteau continue toutefois d'assister, quand il le peut, aux séances de la Société, fait entrer au musée $100 \mathrm{~kg}$ de fiches en fer de l'oppidum de Vue ${ }^{146}$ et un torque en or provenant de Nozay, par exemple. En 1880, après communication à

143. Ibidem, dans les Addenda et errata, p. 137.

144. BSANLI, 18, 1879, p. 8-9, 21 janvier 1879.

145. À cette même séance du 21 janvier 1879, puis à la suivante le 4 février (p. 11-17), Héracle Olivier de Wismes présente à la Société les 37 plus belles pièces ou ensemble d'objets de sa collection qui ont été exposés au Trocadéro, quai de Billy.

146. BSANLI, 18, 1879, p. 18, 18 février 1879. 
la Société ${ }^{147}$, il publie son dernier article sur "Le culte du veau d'or dans l'Antiquité et jusqu'à nos jours ${ }^{148}$ ", mais sans les planches qui, pourtant, sont imprimées. Le musée conserve au moins quatre ou cinq versions différentes et manuscrites de cette publication dont le premier titre a été "Le culte $d u$ veau d'or depuis la chute de l'homme jusqu'à nos jours ". Cette même année, il publie également "Les bijoux bretons ", dans La Bretagne artistique, pittoresque et littéraire, 1880 . Ce seront ses deux dernières publications, même s'il a laissé à l'état de manuscrit, et très inachevé, un nouveau Catalogue complet de ma collection archéologique ${ }^{149}$, rédigé en janvier 1881. Après une note préliminaire relative à son parcours depuis sa Vendée natale jusqu'à sa volonté de faire le don de ses collections au Département, il classe ses collections comme à son habitude, en séries : bagues et anneaux (127 pièces), boucles, agrafes et bijoux (100 pièces), bijoux divers (rouelles surtout, 25 pièces), boutons (22 pièces), reliquaires et bijoux religieux (11 pièces), bracelets (23 pièces), armes antiques (30 pièces), clefs antiques (17 pièces), cheval (24 pièces), lances antiques (17 pièces), haches de l'âge de la Pierre (7 pièces), puis le catalogue s'achève simplement sur le titre " haches polies". Fortuné Parenteau l'a illustré de sa main, en dessinant à la plume dans le texte (ou parfois redessinant) la plupart des objets cités. En outre, en fin de volume, il dessine quelques ensembles récemment découverts, comme les principales pièces du dépôt métallique de la Prairie de Mauves, à Nantes, trouvé à la fin de 1881. Il commence le Catalogue descriptif des monnaies gauloises de ma collection et s'arrête au numéro 8 , inscrit les dernières acquisitions faites pour le musée lors du dernier trimestre de 1881 (monnaies, armes africaines, montre en or de la duchesse de Berry, etc.), enfin il récapitule les documents nécessaires au don de sa collection au Département et colle les coupures de presse relatives à ce don (février 1882).

Les ressources financières du musée semblent de plus en plus réduites et la chapelle de l'Oratoire est complètement envahie d'objets divers car Parenteau, comme conservateur, n'a effectué aucun tri et accepte tous les dons. À tel point qu'à la fin de 1880, le président de la Société archéologique, le baron de Wismes ne peut faire l'acquisition de fragments de sculptures antiques, ornées de "moulures, de denticules et de triglyphes", découverts rue Saint-Jean et dessinés par Louis Petit :

"Il est certain que si le musée pouvait disposer d'un local plus vaste, d'une cour, ou, à défaut de mieux, d'un chantier où seraient déposées les pièces un peu trop encombrantes pour la salle des collections, peu à peu ces tronçons de colonnes, de frises et d'entablements que l'on découvre autour de notre vieille enceinte, formeraient une riche carrière et, peut-être, un jour verrions-nous sortir de cet entassement, ces temples d'Apollon et de Jupiter que nos vieilles chroniques retracent encore à nos regrets ${ }^{150}$. "

147. Le texte lu à la séance de la Société est conservé au musée Dobrée, fonds Parenteau, inv. 997.11.22 (1 et 2).

148. BSANLI, 19, 1880, p. 18-19, 6 avril 1880, et p. 183-186.

149. Musée Dobrée, fonds Parenteau, inv. 997.11.5.

150. BSANLI, 19, 1880, p. 28, 2 novembre 1880. 
La dernière séance de la Société à laquelle Fortuné Parenteau participe est celle du 7 décembre 1880 .

Un an et demi plus tard, le Bulletin de la Société ne mentionne même pas le don au musée de la collection de Parenteau, pourtant largement relaté dans la presse locale ${ }^{151}$. Le conservateur donateur avait rédigé à ce sujet deux courriers officiels ${ }^{152}$ dont l'un, adressé à Louis Herbette, préfet, président de la Commission administrative du musée départemental d'Archéologie, le prie " de vouloir bien offrir, de ma part, ma collection, au Conseil général du département de la Loire-Inférieure ", et l'autre, est adressé au maire de Nantes par le "conservateur du musée départemental et de la Ville de Nantes d'Archéologie de l'Oratoire, avec prière de la communiquer à la commission de la Société archéologique et de plaider ma cause comme membre du Conseil municipal près de l'administration ". Si nous n'avons aucune trace de la réaction du Département à ce don, en revanche, le maire, Georges-Évariste Colombel, en informe le Conseil municipal ${ }^{153}$ qui entérine le don "à la Ville de Nantes et au Département " avec les deux conditions fixées par Parenteau : la collection restera déposée à perpétuité au musée départemental d'Archéologie; la Ville fera confectionner à ses frais les vitrines destinées à recevoir les objets qui composent cette collection ${ }^{154}$.

L'intermédiaire de cette transaction n'est autre que le président de la Société archéologique, Marie-Charles-Jules de La Laurencie ${ }^{155}$. Le don n'est accompagné d'aucune liste; l'Inventaire archéologique de 1878 a fait office de descriptif de la collection. Quelques mois plus tard, le 10 septembre $1882^{156}$, Fortuné Parenteau décède à Pouzauges (Vendée)

151. Articles dans l'Union Bretonne, le Phare de la Loire et l'Espérance du Peuple, du 25 février 1882. Très amer, Fortuné Parenteau écrit «Le Musée de l'Oratoire depuis près de trente ans de direction est mon neveu d'adoption. Je lui fais épouser ma fille et lui donne ma collection. Je partirai pour la Vendée le lendemain de l'installation de ma collection au Musée et ne rentrerai pas à Nantes " : musée Dobrée, fonds Delacoux, 1999, boîte F, n²23, cahier manuscrit.

152. Musée Dobrée, fonds Parenteau, inv. 997.11.5, p. 261.

153. Délibérations du conseil municipal du 23 février 1882.

154. Le Conseil municipal vote un crédit de $1500 \mathrm{~F}$ pour la confection des vitrines (Délibérations).

155. Avec le baron Héracle Olivier de Wismes, Charles de La Laurencie a songé à organiser une exposition à l'occasion de ce don. Fortuné Parenteau, qui, toute sa vie, a rêvé d'une grande exposition archéologique à l'Oratoire, en est très fâché car, comme toujours, l'exposition serait plutôt une exposition d'" art décoratif ", illustrée par les collections des collectionneurs locaux et, de plus, ne se tiendrait pas au musée de l'Oratoire. Parenteau écrit donc avant son départ définitif en Vendée que "Ici à Nantes, les deux dernières expositions d'archéologie ont été organisées à la Monnaye et au Foyer du théâtre de la Renaissance; personne n'aurait voulu l'installer au Musée de l'Oratoire. Une exposition comme celle qu'ils rêvent est ridicule, mesquine... Mais si l'exposition n'a pas lieu, Mrs de La Laurencie et de Wismes ne me le pardonneront pas ", musée Dobrée, fonds Delacoux, 1999, boîte $\mathrm{F}, \mathrm{n}^{\circ} 23$, cahier manuscrit.

156. Acte de décès aux Archives départementales de la Vendée, état civil de Pouzauges, Décès, AD2E182/14, années 1870-1883. Il est toujours inscrit comme "propriétaire " dans l'acte de décès. 
"à la suite d'une longue et pénible maladie, qui, jusqu'au dernier instant, lui laissa la pleine jouissance de sa belle intelligence et de son noble coeur $^{157}$ ". Pitre de Lisle du Dreneuc ajoutera :

«Épuisé par la terrible maladie qui devait nous l'enlever, [...] il lutta énergiquement contre le mal, et, jusqu'au dernier jour, il remplit avec le plus grand zèle les devoirs de sa charge [...] et, peu d'heures avant sa mort, il étudiait certains objets anciens qu'il destinait aux collections départementales et que lui apportait son fidèle Thomas, gardien depuis vingt-cinq années, du musée de l'Oratoire ${ }^{158}$. "

Dans un document manuscrit et non daté conservé au musée Dobrée, Pitre de Lisle précise :

" Il était presque mourant lorsque courageux jusqu'au bout, il vint luimême apporter sa collection au Musée et c'était vraiment touchant de voir ce collectionneur passionné se séparer de ses chers trésors et les laisser de ses mains tremblantes dans ce musée qu'il ne devait plus revoir [...]. Aidé de Madame Parenteau, il apportait de la rue Bertrand-Geslin à l'Oratoire, dans des boîtes dans ses poches, sur les coussins de la voiture, les superbes bibelots qu'il installait dans les vitrines que l'on venait de terminer ${ }^{159}$. "

Peu avant sa mort, Parenteau a songé à prendre Thomas Dobrée comme exécuteur testamentaire.

En 1883, Madame Parenteau donne au musée " une certaine quantité de livres traitant de l'archéologie et de numismatique " provenant de la bibliothèque de son mari ${ }^{160}$ (fig. 37). Après le décès de Madame Parenteau, ce qui restait des collections de son mari est vendu en vente publique les 30 juin et $1^{\text {er }}$ juillet 1902. C'est Paul Soullard (parfois écrit Soulhard), luimême numismate, ami de très longue date de Fortuné Parenteau, bibliothécaire-archiviste de la Société archéologique de Nantes et membre de la Commission administrative du musée, qui rédige le catalogue de la vente ${ }^{161}$ et l'archéologue poitevin Gustave Chauvet, beau-frère de Parenteau, écrit la notice sur le défunt ${ }^{162}$. Quelques objets archéologiques, surtout des bijoux, figurent au catalogue qui comporte 364 numéros ou lots. L'ensemble met en évidence l'importance des collections de Parenteau qui touchent à de nombreux domaines : souvenirs de la duchesse de Berry, objets décoratifs (arts de la table) du XvIII ${ }^{\mathrm{e}}$ siècle, éventails, verres vénitiens, faïences, porce-

157. BSANLI, 22, 1883, p. 204, " Nécrologie, M. F. Parenteau ", signée "Un ami ».

158. Lisle du Dreneuc, Pitre de, «M. Fortuné Parenteau, conservateur du Musée de l'Oratoire à Nantes ", $R B V, 1883$, p. 143.

159. Musée Dobrée, archives collectionneurs, s.v. Parenteau, Pitre de Lisle du Dreneuc, Collection Parenteau, vers 1902. En fait, il s'agit sans doute d'une partie du rapport que Pitre de Lisle rédige en 1902 sur la collection Parenteau à la demande de la Commission administrative du musée départemental d'Archéologie.

160. Archives du musée Dobrée, lettre du préfet Anatole Catusse à Pitre de Lisle du Dreneuc, 13 août 1883.

161. Catalogue pour la vente d'objets anciens ayant fait partie des collections de Fortuné Parenteau, Fontenay-le-Comte, Charles Claireaux impr., 1902, musée Dobrée, fonds Delacoux. 1999, boîte F, 23.

162. Ibidem, p. III à v. 
laines, gravures, médailles, monnaies (gauloises, féodales, royales), objets extra-européens, etc.

En tant que président de la Société archéologique, Héracle Olivier de Wismes dresse un bref éloge funèbre de Fortuné Parenteau lors de la séance du 7 novembre 1882. Pour la première fois, est évoqué le don du conservateur :

"Nous avons à déplorer la mort de notre savant et si dévoué collègue, M. Fortuné Parenteau, dont chacun connaissait le zèle et l'attachement à la Société [...]; en prononçant de nouveau son nom, c'est, je crois, le meilleur souvenir que je vous puisse laisser de ce bon collègue dont la dernière pensée fut pour nous, et dont nous sommes heureux d'admirer avec reconnaissance, dans nos vitrines, la magnifique collection ${ }^{163}$."

Une nécrologie anonyme, signée "Un Ami », paraît l'année suivante dans le Bulletin de la Société archéologique :

"La mort frappait à Pouzauges, le 10 septembre dernier, le Conservateur du Musée départemental d'Archéologie de Nantes, âgé de soixante-neuf ans. C'est là le seul titre qu'ambitionnait M. F. Parenteau [...]. Il n'avait qu'un but, qu'une préoccupation, l'étude et son Musée de l'Oratoire ${ }^{164}$."

\section{Pitre de Lisle du Dreneuc (1846-1924)}

\section{Archéologue dilettante et infatigable "découvreur" de mégalithes}

Le manque d'archives relatives à Pitre de Lisle du Dreneuc ne permet guère de retracer avec précision le parcours du second conservateur du musée départemental de l'Archéologie de l'Oratoire, premier conservateur du musée Thomas Dobrée. Le vicomte Pierre René (dit Pitre) de Lisle du Dreneuc, naît à Nantes, 6 rue Jean-Jacques-Rousseau, le 24 avril 1846. Il est le cinquième fils de Pierre de Lisle du Dreneuc et de Renée Arsène Bureau ${ }^{165}$. Grâce à la notice nécrologique rédigée par son successeur et ami, le chanoine Georges Durville, dans l'Écho de la Loire, on apprend qu'il est issu " d'une de ces anciennes familles nantaises qui se signalait par leur fidélité aux vieilles traditions bretonnes ${ }^{166}$ ". Son père avait été officier de la garde du Roi sous la Restauration, jusqu'à la chute de Charles X qui provoque sa démission (1830). Son fils Pitre a songé à s'engager parmi les zouaves pontificaux, comme ses frères Georges et Henri, mais son goût pour la chasse l'amène très tôt à découvrir sa passion ${ }^{167}$ : les mégalithes. Comme l'explique le chanoine Durville :

163. BSANLI, 21, 1882, p. IX, 7 novembre 1882.

164. BSANLI, 22, 1883, p. 204-209.

165. Acte de naissance aux Archives municipales de Nantes, état-civil du $5^{\mathrm{e}}$ canton, Naissances, 1E784/1846.

166. DuRVILLE, chanoine Georges, "Nos grandes enquêtes : M. le vicomte P. de Lisle du Dréneuc, conservateur du musée Dobrée et du musée d'archéologie ", L'Écho de la Loire, février 1924.

167. Dans Le Phare de la Loire, "Nécrologie, les obsèques de M. de Lisle du Dréneuc ", le préfet de la Loire-Inférieure, Paul Bouju, écrit à ce sujet : "Rien de son pays ne le laissait 
"L'archéologie sourit de bonne heure au futur conservateur de nos musées départementaux. Dans sa jeunesse, grand amateur de la chasse, il ajoutait à celle du gibier, celle des monuments mégalithiques. [...] En compagnie de ces frères, Arthur et Georges de Lisle qui, partageant ses goûts, partagèrent aussi ses fatigues, il se mit à parcourir la région, arpentant les champs, sondant les bois, fouillant le sol dans l'espérance d'y trouver un souvenir des Gaulois ou des populations qui les ont précédés dans notre pays. "

Pitre de Lisle exprime ce plaisir de la découverte d'un mégalithe : "J'éprouve une émotion de chasseur qui tombe sur une belle pièce de gibier longtemps poursuivi à en découvrir de nouveaux [monuments mégalithiques] ${ }^{168}$. " De sa formation, on ne connaît rien. Il se marie avec Marie Françoise Marguerite Adrienne Espitalié-La Peyrade (1852-1928) dont il a huit enfants entre 1881 et $1895^{169}$ (fig. 38 à 41 ).

En dehors de son goût pour la chasse et la découverte de mégalithes, on ne sait pas comment il fait vivre sa famille, car, contrairement à Fortuné Parenteau qui disposait des revenus de ses terres et fermes vendéennes, Pitre de Lisle ne semble disposer d'aucun bien, à l'exception de sa propriété du Fief à Sautron où il vécut une partie de sa vie (fig. 42 et 43).

Outre le côté "chasseur" de sa quête des monuments mégalithiques, Pitre de Lisle s'inscrit dans un courant d'intellectuels passionnés d'archéologie de la seconde moitié du XIX ${ }^{\mathrm{e}}$ siècle. On n'est plus alors dans la recherche de l'universalité qui caractérisait jusque là les érudits nantais, mais déjà dans une démarche scientifique spécialisée. Ainsi, au Congrès

indifférent. N'avait-il pas entrepris, au temps de ses randonnées intrépides de gentilhomme chasseur, de constituer une collection des oiseaux du pays. Bien d'autres chasses le sollicitèrent par la suite. "

168. Dans le discours de M. Dortel, président de la Commission administrative du musée départemental d'Archéologie, aux obsèques de Pitre de Lisle du Dreneuc, Le Phare de la Loire, 17 février 1924, " Nécrologie, les obsèques de M. de Lisle du Dréneuc ". La première mention que nous possédons de la découverte d'un mégalithe concerne une visite à Batzsur-Mer, mais il est probable qu'il se livra bien plus tôt à ce type de recherche.

169. Il se marie à Nantes, le 6 juillet 1880. Il est alors désigné comme "propriétaire " demeurant à La Haie-Fouassière (Loire-Inférieure). Ses frères, Henri, âgé de 48 ans, et Georges, 39 ans, sont ses témoins. L'acte de mariage conservé aux Archives municipales de Nantes (1E 1431) indique dans la marge une modification de l'orthographe du nom du marié inscrit sur le registre "de L'Isle du Dréneuf " et qu'il faut désormais lire "de Lisle du Dreneuc ", depuis un jugement du 7 avril 1908. 
Figure 38 - Pitre de Lisle du Dreneuc et son épouse Adrienne, assis, au manoir du Fief à Sautron (L.-A., coll. part. - cl. musée Dobrée)

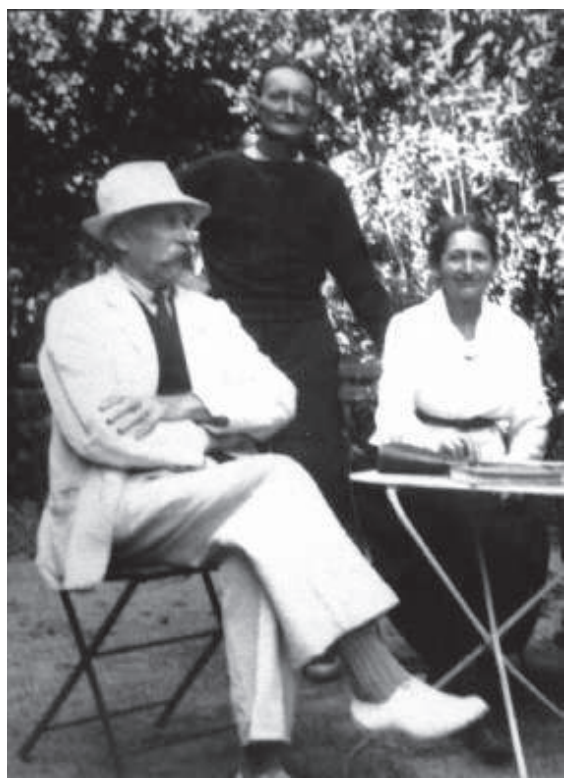

Figure 39 - Adrienne (Madame $P$. de Lisle du Dreneuc), photographie anonyme (coll. part., cl. musée Dobrée)

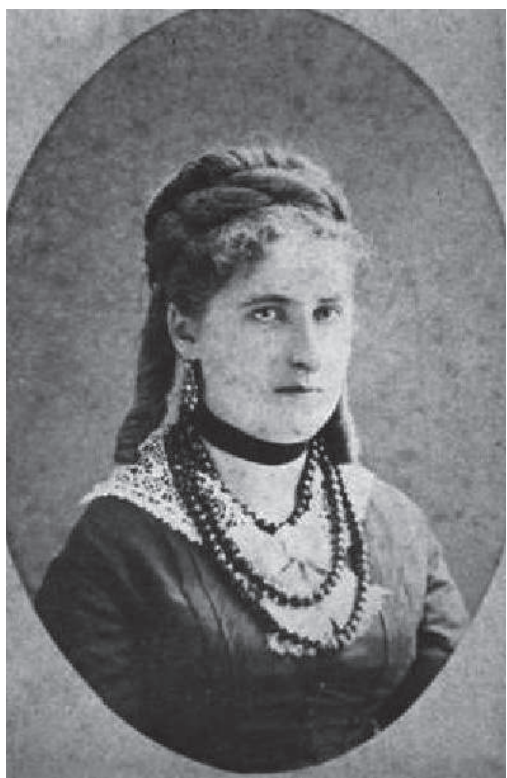

Figure 40 - Les huit enfants de Pitre de Lisle du Dreneuc à La Baule-Escoublac

(L.-A., coll. part. - cl. musée Dobrée)

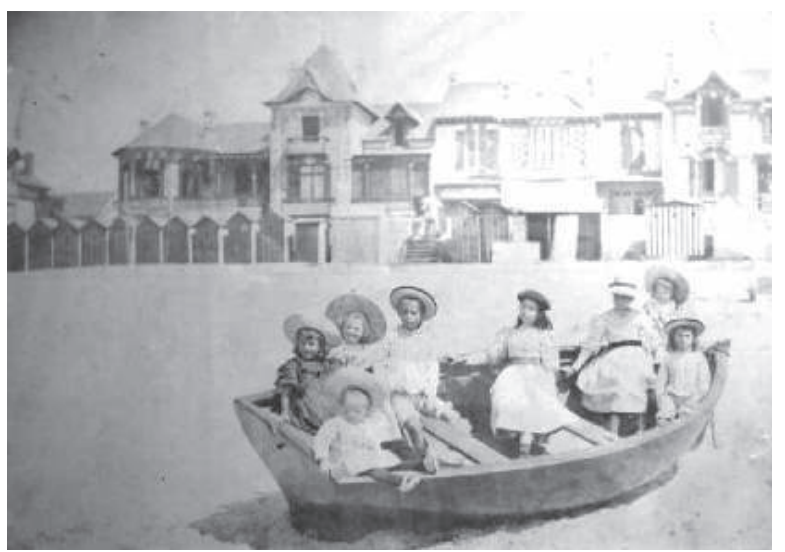


Figure 41 -

Caricature : Pitre de Lisle jouant une berceuse à l'un de ses enfants, anonyme (Paul de Lisle?)

(coll. part. cl. musée Dobrée)
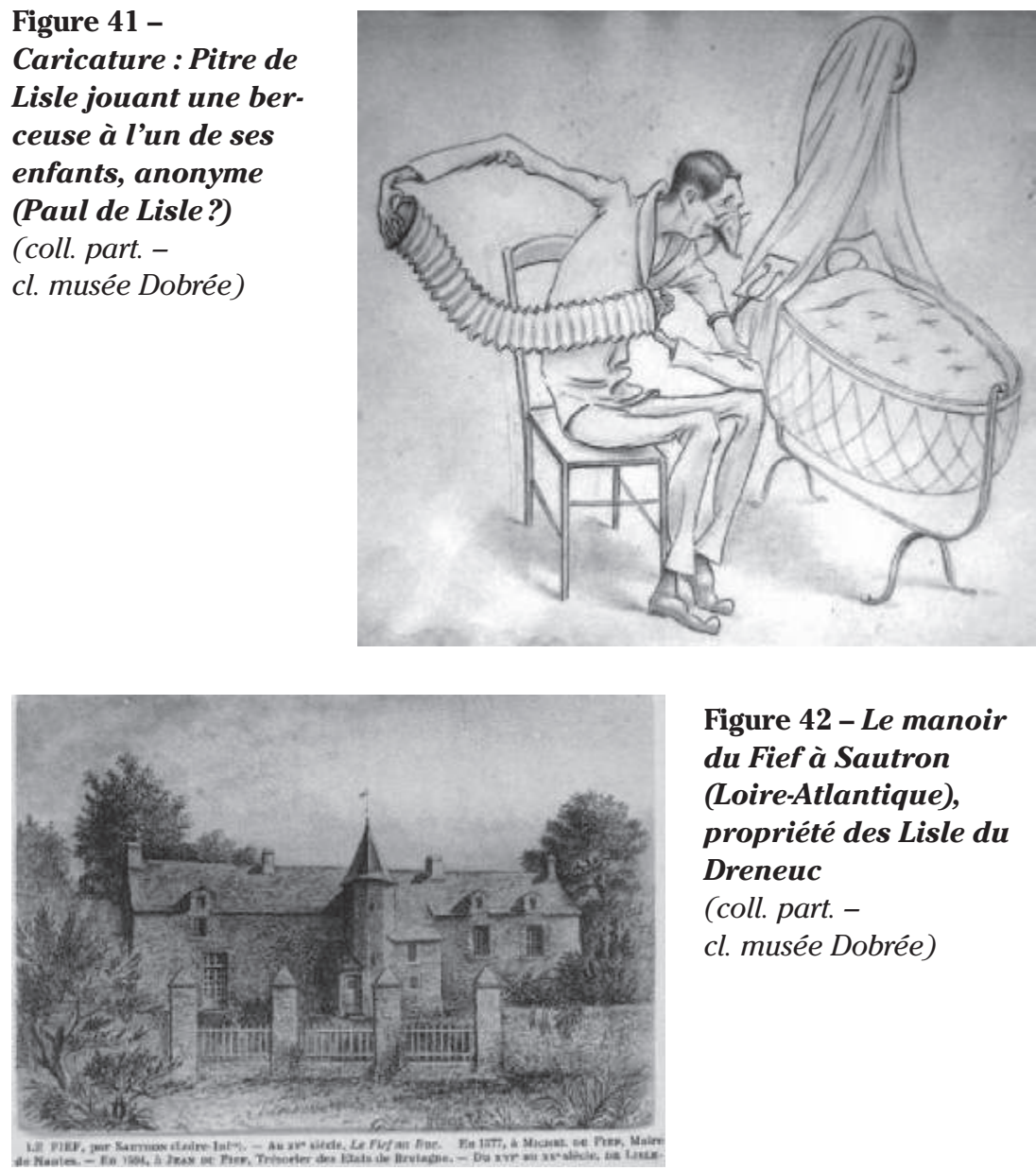

Figure 42 - Le manoir du Fief à Sautron (Loire-Atlantique), propriété des Lisle du Dreneuc

(coll. part. cl. musée Dobrée)

Figure 43 - Le manoir du Fief à Sautron (Loire-Atlantique), par Paul de Lisle du Dreneuc (coll. part. - cl. musée Dobrée)

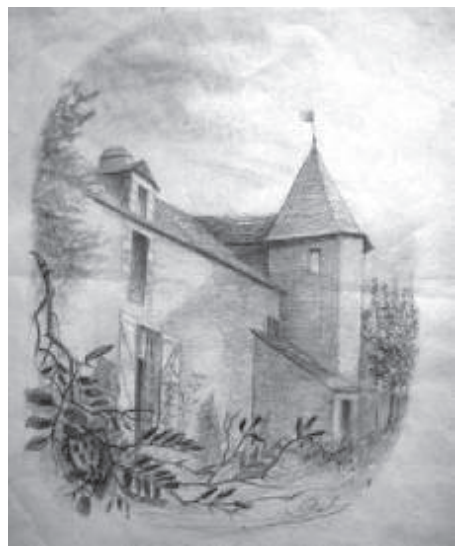


de l'Association française pour l'Avancement des Sciences qui s'est tenu à Nantes en 1875, Gabriel de Mortillet constate "le peu d'industrie paléolithique en Bretagne ${ }^{170}$ ". Quelques années plus tard, en 1882, au Congrès de l'Association bretonne, Pitre de Lisle du Dreneuc répond à Gabriel de Mortillet en publiant les Stations primitives de la Bretagne, dont deux sites paléolithiques majeurs, le Mont Dol, à Dol (Ille-et-Vilaine) et les "stations" de Montbert, à côté du lac de Grand-Lieu (Loire-Atlantique) ${ }^{171}$.

C'est à plus de cinquante ans, à la fin de l'année 1877, que Pitre de Lisle du Dreneuc (inscrit sous la graphie de Pitre de L'Isle du Dréneuf), pourtant membre de la Société archéologique depuis le 28 octobre $1872^{172}$, prend part pour la première fois aux séances de la Société. Il communique sur les découvertes de "stations" préhistoriques en Loire-Inférieure (L'Étranglard à Saint-Géréon, La Haie-Pallet à Mouzillon, Le Rocher et Bégrol à La HayeFouassière, enfin La Canterie ou le Clos de Moquechien à Saint-Fiacre) ${ }^{173}$, qu'il publie en 1878, comme "résultat des explorations que nous avons faites, mon frère Georges de l'Isle et moi ${ }^{174 " . ~}$

Dès la fin de l'année 1877, Pitre devient secrétaire du comité de la Société archéologique sous la présidence du baron Héracle Olivier de Wismes, autre passionné des sites néolithiques. Il s'atèle très rapidement à la grande publication qu'il va poursuivre jusqu'en 1885, mais restera inachevée : L'Inventaire archéologique de la Loire-Inférieure, en commençant par l'arrondissement de Châteaubriant, qu'il présente à une séance de la Société ${ }^{175}$. Il en publie une première partie dans le Bulletin de $1880^{176}$. Pour ce faire, et grâce à un esprit méthodique et à un sens aigu de l'observation, il réalise un grand travail de compilation, de vérification de sources, de recherches personnelles et met au point une méthodologie de publication encore inusitée dans les publications locales, avec, groupée par commune, depuis l'époque préhistorique jusqu'à la fin de l'occupation romaine, l'ensemble des découvertes connues ${ }^{177}$. La localisation précise des sites sur le cadastre (section et parcellaire), la création d'icones (signes conventionnels) pour identifier les types de monuments,

170. Conservateur du musée des Antiquités nationales de Saint-Germain-en-Laye et fondateur de la revue Matériaux pour l'Histoire primitive et naturelle de l'Homme (= Matériaux).

171. Bulletin archéologique de l'Association bretonne (=BAAB), 1882, p. 3-27.

172. BSANLI, 11, 1872, p. 151. Pitre est présenté par Charles Marionneau et le militaire Charles Bougouin, membres résidants de la Société.

173. BSANLI, 16, 1877, p. 213-214, 4 décembre 1877.

174. LiSLE DU DRENEUC, Pitre de, "Stations paléolithiques et néolithiques de la LoireInférieure ", BSANLI, 17,1878, p. 45-56. Cet article est immédiatement salué par la communauté scientifique, voir Matériaux, IX, 1878, p. 522-525.

175. BSANLI, 19, 1880, p. 24-25, $1^{\text {er }}$ juin 1880.

176. LISLE DU DRENEUC, Pitre de, « Dictionnaire archéologique de la Loire-Inférieure (époques primitive, celtique, gauloise et gallo-romaine), arrondissement de Châteaubriant ", BSANLI, 19, 1880, p. 117-182.

177. En fait, il applique la même méthode que Gaston de La Chenelière pour le nord de la Bretagne. 
etc., facilitent la lecture de son Dictionnaire. Il explique sa méthode dans l'introduction du Dictionnaire :

" Nous avons dressé une liste alphabétique des communes, en indiquant pour chacune d'elles les monuments, les trouvailles, les stations et les retranchements en terre de l'époque primitive. Les lieux dits dont les noms peuvent aider à faire connaître des points intéressants, des mégalithes, des tombes, des cachettes, ont été notés sur les matrices cadastrales, et transcrits à la suite des indications archéologiques. Enfin, j'ai cru devoir citer les antiquités gallo-romaines, stations, voies, castrum, etc. Les objets et les monuments de cette époque sont si intimement mêlés aux vestiges des époques antérieures, qu'il est difficile de les séparer tout à fait ${ }^{178}$. "

Pitre de Lisle publie trois arrondissements du nord de la Loire (Châteaubriant, Saint-Nazaire et Paimbœuf) mais ne peut achever ce travail au sud de la Loire ${ }^{179}$. Pour faciliter la lecture de son dictionnaire, il choisit une présentation alphabétique des sites et réalise un tableau de synthèse des découvertes ${ }^{180}$. Bien que reconnu comme fort intéressant et essentiel, cet inventaire fait l'objet d'une critique de Paul du Chatellier et, surtout, d'Émile Carthailhac ${ }^{181}$. Ce dernier déplore le manque d'illustrations, le fait que Pitre de Lisle n'ait pas exposé la question de la Brière et, enfin, remet en cause la datation proposée par l'auteur pour les monuments mégalithiques :

"M. Pitre de Lisle semble croire que l'archéologie pure ne reconnaît pas aux dolmens de l'Ouest une antiquité plus grande que le $v^{\mathrm{e}}$ siècle avant notre ère, et il laisse à ce qu'il appelle " une certaine école, les vues grandioses " touchant l'ancienneté de l'époque du renne. J'en appelle pour regretter cette ligne à M. Pitre de Lisle mieux informé ${ }^{182}$. "

Pitre tient deux carnets de ses excursions, malheureusement incomplets mais précieux ${ }^{183}$. Le premier, conservé en collection particulière, est

178. Ibidem, p. 119.

179. Le chanoine Georges Durville le regrette dans l'éloge qu'il fait de Pitre de Lisle du Dreneuc : «La façon remarquable dont il a traité son sujet fait regretter plus vivement qu'il n'ait pas achevé son ouvre. Il y a là, pour la Préhistoire du sud de la Loire, une lacune difficile à combler " (L'Écho de la Loire, février 1924).

180. Après l'arrondissement de Châteaubriant, il publie celui de Saint-Nazaire, BSANLI, 20, 1882 , p. $93-176$ ( $1^{\text {re }}$ partie) et $B S A N L I, 21,1883,38-118$ ( $2^{\mathrm{e}}$ partie), puis celui de Paimboeuf, $B S A N L I, 24,1885$, p. 1-81. En 1882, il rassemble les trois arrondissements de Châteaubriant, Saint-Nazaire et Paimbœuf : Dictionnaire archéologique de la Loire-Inférieure (époques primitive, celtique, gauloise et gallo-romaine) : arrondissements de Châteaubriant, SaintNazaire et Paimboeuf (Dictionnaire archéologique), Nantes, 1882, Vincent Forest et Émile Grimaud imp.

181. " Dictionnaire archéologique de la Loire-Inférieure ", dans Matériaux, 1882-1883, p. 215-216 et 1885, p. 121-124.

182. CARTAILHAC, Émile, dans Matériaux, 1885, p. 124.

183. Archives privées, "Hommage au vicomte Pitre de Lisle du Dreneuc ", anonyme, s.d. (1924?) : "Il parcourt chaque commune interrogeant toutes les personnes susceptibles de lui apporter des renseignements. Combien de kilomètres dut faire le vicomte de Lisle au pas lent de son cheval trainant le break?" 
un journal où il relate au jour le jour, de mai 1877 à novembre 1887, ses occupations, ses impressions, le temps qu'il fait et bien d'autres choses ${ }^{184}$ :

"Ce jour, premier de mai [1877], j'ai commencé le présent cahier. Ainsi que dans le registre de M. de la Meilleraye, je veux noter chaque jour les déplacements, faits mémorables et autres de peu d'importance qu'il me plaira de retrouver ${ }^{185}$."

Ainsi, par exemple, entre le 11 et le 16 mai 1877, Pitre écrit :

"11 Excursion à Bégrolle et par La Ramée, où le veau est né dans la nuit, au-dessus de La Thébaudière. Flèche en silex tranchante.

12 Je fus à Nantes avec Georges, arrêter un appartement. Léon projette un voyage d'explorations pour découvrir la frontière de la langue bretonne et les différents dialectes.

13 Dimanche. De Bégrolle nous coupons par La Petière à La Canterie.

14 Le Breil et Bégrolle, moins rapidement et sans résultat. Le soir, je dînai à La Giraudière.

15 Mardi. Je fus avec Pierre Patelin, visiter les fouilles de Kerviler à St-Nazaire. L'anse de Penhouet a été barrée par une jetée formée par les terres que l'on a retirées du $1^{\text {er }}$ bassin commencé en 1842. À l'époque galloromaine, cette anse servait peut-être de port. En tout cas, des poteries et une monnaie de Tetricus ont été trouvées dans une couche de sable et de galets. La couche de vase entre les niveaux de 1842 et l'époque de Tetricus donne une série de couches séparées dont le nombre correspond avec le nombre approximatif d'années écoulées soit, environ, 1550, Tetricus régnant en 270. En creusant à une profondeur plus grande, Kerviler a trouvé une épée en bronze, un poignard, des poteries en terre noirâtre, très grossières de forme et ressemblant à des fonds de bouteilles à cidre, une corne de cerf forée pour le passage d'un manche et creusée à chaque bout pour emmancher une hachette d'un côté, une pointe, de l'autre. Ces objets appartiennent évidemment à l'âge préhistorique de ce point de notre pays. Voulant fixer la date de cette période, Kerviler a repris les hauteurs des dépôts laissées par un siècle, soit $35 \mathrm{~cm}$, et a retrouvé un laps de temps de 3000 ans environ, entre l'époque de Tetricus et celle de ces armes. Les objets n'ont pas été trouvés superposés mais il est facile de suivre le niveau romain parfaitement tranché, jusqu'au-dessus du puits et du fond où l'on rencontre les instruments primitifs. Une énorme vertèbre que l'on vient de trouver dans le puits creusé spécialement pour ses fouilles, va peut-être nous conduire à la date de l'homme des cavernes. Kerviler l'a immédiatement emballée et emmenée à Paris.

Tous les objets trouvés sont installés dans un musée provisoire : une corne de cerf porte la trace d'une coupure circulaire pour en détacher une pointe. Deux cornes de chevreuil, noires comme de l'encre, une aiguille en os complètent sa collection. D'énormes pierres percées de trou et ayant servi d'ancres prouvent qu'à l'âge du Bronze, l'anse servait de port. [...] Au temps où le niveau des vases se trouvait au-dessus des basses marées, les dépôts ont dû être bien plus abondants; la régression plus forte compense cette

184. Archives privées, Pitre de Lisle du Dreneuc, Journal, 1877-1887 (copie numérisée, archives du musée Dobrée).

185. Pitre de Lisle du Dreneuc, Journal, p. 1. 
plus grande épaisseur. Toutes ces circonstances ont été pesées et appréciées par notre ingénieur.

Le soir, il nous a accompagnés jusqu'à Nantes, et de là, a gagné Paris. Sa femme est une $\mathrm{D}^{\text {lle }}$ Guyesse de Lorient, nièce de ce peintre à qui Briseux a dédié le poème Marie.

16 Insipide promenade à la recherche du Fief de Loiselinière. Le soir, je fus voir le vicaire."

Parfois, Pitre illustre ses notes de croquis, de plans, de dessins aquarellés de mégalithes ou d'objets, de paysages ou de monuments (fig. 44-47), parfois encore il donne dans l'ironie (fig. 48).

Figure 44 - Gourmelon (Pornic, L.A.), Journal de Pitre de Lisle du Dreneuc, 5 septembre 1877 (coll. part. - cl. musée Dobrée)

Figure 45 - La Vrillère à La Chapelle-Basse-Mer (L.-A.), Journal de Pitre de Lisle du Dreneuc, 30 juin 1879 (coll. part. - cl. musée Dobrée)
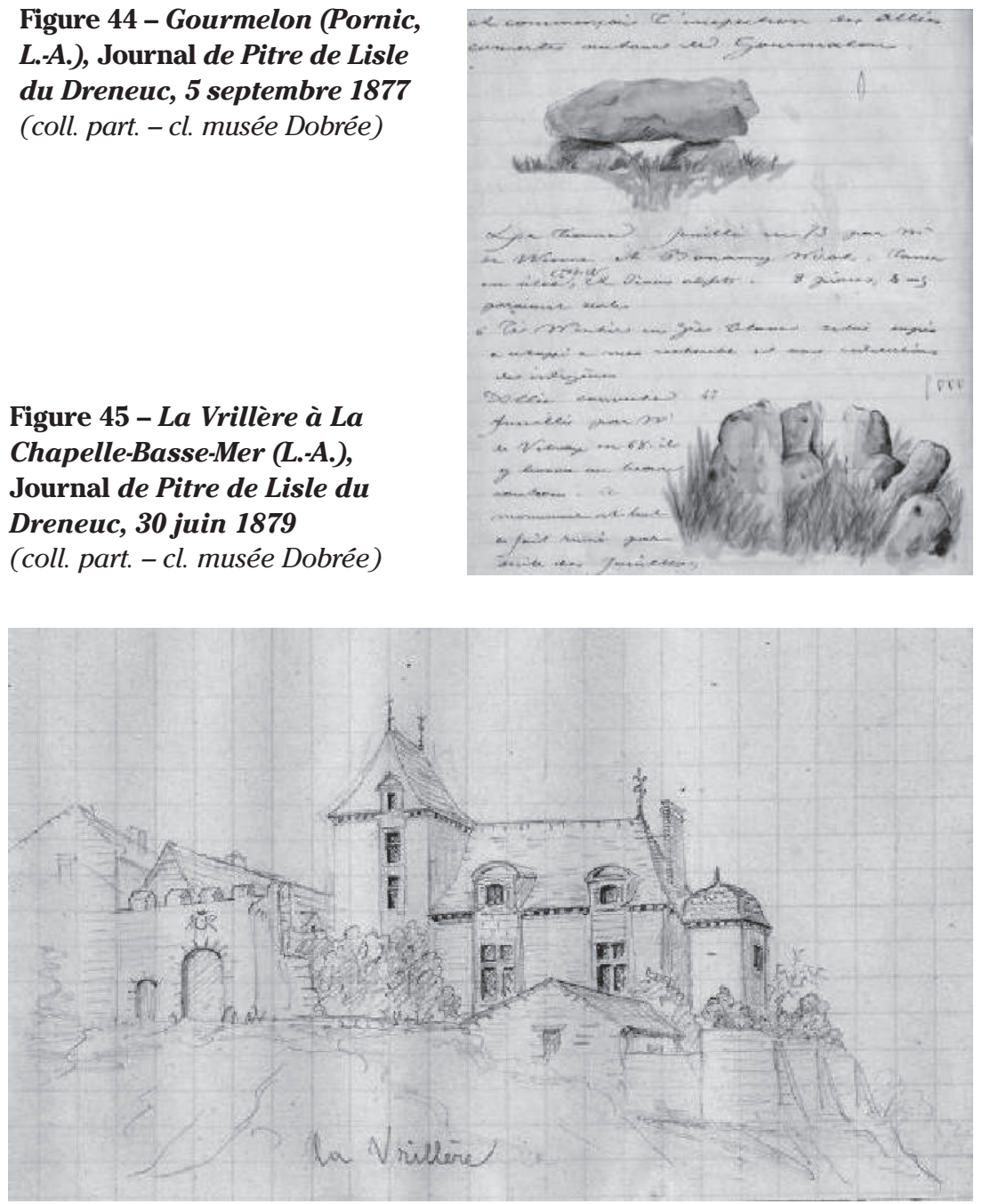


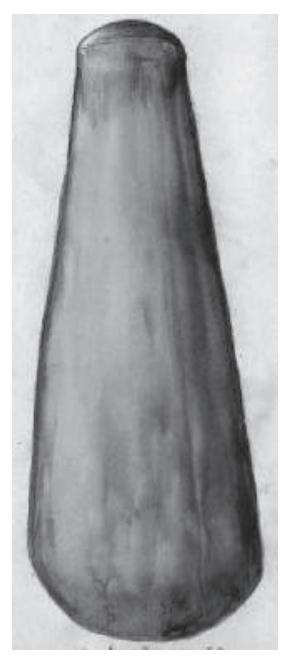

Figure 46 - Hache à bouton de Vallet (L.-A.), Journal de Pitre de Lisle du Dreneuc, 27 mai 1878

(coll. part. - cl. musée Dobrée)

Figure 47 - Coupe stratigraphique des fouilles de Georges et Pitre de Lisle du Dreneuc à Montbert (L.A.), Journal de Pitre de Lisle du Dreneuc, 18 juin 1878 (coll. part. - cl. musée Dobrée)
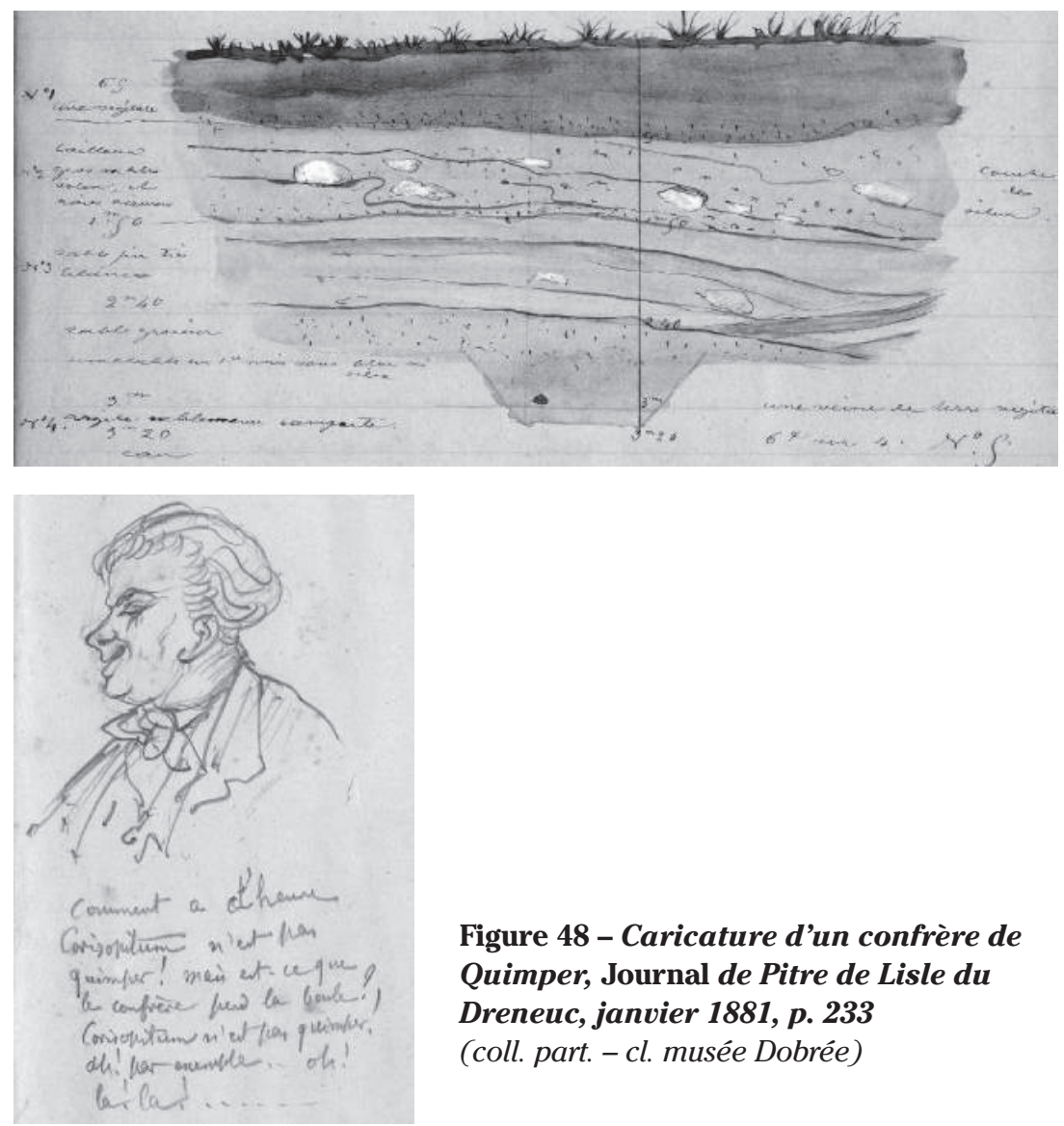

Figure 48 - Caricature d'un confrère de Quimper, Journal de Pitre de Lisle du Dreneuc, janvier 1881, p. 233

(coll. part. - cl. musée Dobrée) 
Le second carnet, intitulé Notes de voyage de Pitre de Lisle du Dreneuc, est un inventaire alphabétique des sites visités entre 1850 et $1893^{186}$. Sans doute conçu au départ pour faciliter la rédaction de son Dictionnaire archéologique, il est très incomplet et ne comporte que deux dessins de mégalithes, sur calque (fig. 49).

\section{Figure 49 - Menhir \\ en grès de la \\ Courrasserie à \\ Doulon (Nantes, \\ L.A.), Pitre de Lisle du Dreneuc, Notes de voyage \\ (coll. part. - \\ cl. musée Dobrée)}

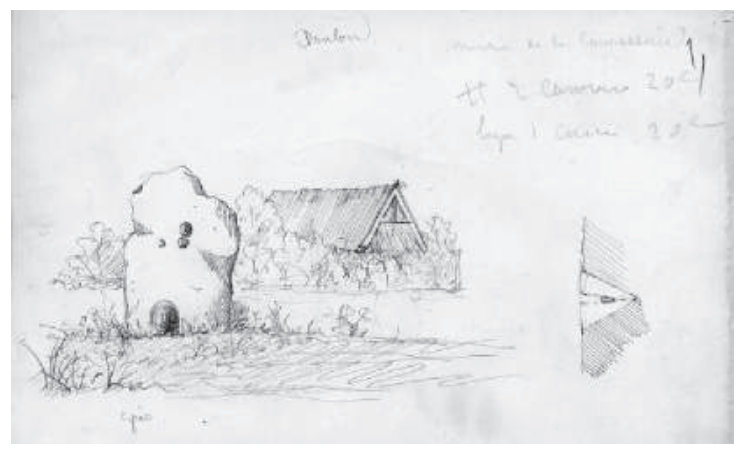

À Rezé (L.-A.), par exemple, où il note des découvertes entre 1850 et 1876 , il indique :

« 1850 Un portique à colonnes, façade de temple ou de palais, découvert en 1850

1851 Dans les travaux creusés pour la construction du Pont de Rezé, on découvrit des poteries, des meules, des monnaies, etc.

1857 À $8 \mathrm{~m}$ de l'église, on découvrit une salle de $8 \mathrm{~m}$ sur 3 avec des restes de mosaïques, poteries, etc.

1858 On découvrit une $2^{\mathrm{e}}$ salle contigüe à la première qui était ornée d'arcatures dessinées en petits carreaux noirs [...].

1859 Dans les travaux de voierie, on découvrit à Rezé 4 colonnes brisées, bases, fûts et chapiteaux. Une hache brisée et 20 sarcophages [...].

1872 Des fouilles dirigées par Monsieur Marionneau dans l'enceinte du prieuré de Saint-Lupien ont mis au jour : 1. une muraille gallo-romaine et la chapelle du XVème; elle est appareillée en opus spicatum. 2. de nombreux sarcophages en calcaire coquillier et pierre de Crazanne; ils contiennent des squelettes, un vase en terre grossière renfermant encore des charbons, des fragments de poteries gallo-romaines et quelques pièces de monnaie assez frustes.

1876 Une Vénus en médaillon, donnée par M. de Monti et une petite lampe avec le poisson [...]."

Pendant 12 ans, entre 1880 et 1892, l'activité de Pitre de Lisle du Dreneuc est intense et les publications scientifiques s'enchaînent, principalement sur le sujet qui le passionne : la pré- et la protohistoire. Infatigable "inven-

186. Musée Dobrée, archives, fonds de Lisle du Dreneuc. Il semble avoir été rédigé entre 1850 et 1893 . 
teur" de mégalithes, il arpente avec son frère Georges, non seulement le département mais toute la Bretagne ${ }^{187}$. Il en résulte une production écrite considérable. Pour le Paléolithique et le Néolithique, il décrit avec méthode les "stations" préhistoriques de la Bretagne et du département ${ }^{188}$, donnant des plans et des coupes, précisant les matériaux utilisés, la nomenclature des outils, etc. Il fouille les tumulus de La Roche à Donges ${ }^{189}$, décrit les menhirs du pays de Retz ${ }^{190}$, les mégalithes du pays Guérandais ${ }^{191}$, de SaintMichel-Chef-Chef ${ }^{192}$ et les tumulus de Pornic ${ }^{193}$. Certains mégalithes ont aujourd'hui disparus, comme le dolmen du Moulin Perret à Corsept, fouillé en 1883, mais ils peuvent être étudiés grâce aux notes précises et aux croquis qu'il en a laissés. Pitre s'intéresse également au mobilier découvert, particulièrement aux haches polies et aux armes de l'âge du Bronze ${ }^{194}$ et, pour comparaison, s'intéresse aux découvertes étrangères à l'Armorique (fig. 50 et 51). Il établit des typologies, et ses descriptions des objets comme des lieux de découvertes sont précises et restent utiles. Il dessine sans doute moins que Parenteau et davantage les sites et monuments que les objets mais, jusqu'à ce que le musée Dobrée ne retrouve plus d'un siècle plus tard le mobilier chalcolithique du tumulus de La Roche, à Donges,

187. BSANLI, 20, 1881, "Allocution de M. le baron de Wismes, président sortant ", p. XxxVII : "Avec M. de Lisle du Dréneuf nous poursuivons de bien neuves et curieuses investigations dans le domaine du préhistorique. Non seulement il fouille des dolmens vierges et jusqu'à lui presque inconnus, mais il semble que notre collègue ait comme un don de double vue pour retrouver dans nos contrées les traces des populations qui ont précédé celles des dolmens et ne se servaient encore que de la pierre non polie."

188. LisLE Du Dreneuc, Pitre de, "Stations paléolithiques et néolithiques de la LoireInférieure ", $B S A N L I, 17,1878$, p. 45-56. " La Bretagne primitive ", $B A A B, 1881$, p. 51-84, suivi des "Stations primitives de la Bretagne ", 1882, p. 3-27.

189. Lisle du Dreneuc, Pitre de, "Fouilles du tumulus de la Roche, Donges, LoireInférieure ", BSANLI, 20, 1881, p. 75-88.

190. LisLe Du DRENEUc, Pitre de, "Les triangles de menhirs de la Loire-Inférieure et les menhirs triangulaires du Pays de Retz ", $R A, 1886,1$, p. 139-144.

191. LisLE Du DRENEUC, Pitre de, « Les fouilles du grand monument de Boga en Guérande (Loire-Inférieure) ", BSANLI, 29, 1890, p. 162-165.

192. LisLE Du DRENEuc, Pitre de, "Les dolmens de Saint-Michel-Chef-Chef ", BSANLI, 25, 1886, p. 127-134, et " Fouilles des dolmens du Grand Carreau Vert, Saint-Michel-Chef-Chef ", Matériaux, 1886, 3, p. 277-285. Pitre de Lisle complète la fouille du tumulus par le baron de Wismes en 1875 et y découvre un septième dolmen, celui du Grand Carreau Vert, qui a conservé son mobilier.

193. Lisle du Dreneuc, Pitre de, « Notice sur les fouilles du tumulus de La Motte, SainteMarie (Loire-Inférieure) ", BSANLI, 31, 1892, p. 183-203.

194. LISLE DU DRENEUC, Pitre de, "Caractères particuliers des antiquités primitives de la Bretagne, $B A A B, 1880$, p. 113-118; id., " Notes sur les différentes armes de pierre et de bronze, trouvées aux environs de Donges (Loire-Inférieure) ", BSANLI, 20, 1881, p. 89-90; $i d$., " Découvertes de haches en plomb (Bretagne) ", RA, déc. 1881, p. 335-343; id., Les haches à tête de la Bretagne et de Vendée, Vincent Forest et Émile Grimaud, 1881; id., "Les armes de bronze du sud-est et du nord-est de la Bretagne ", $B A A B, 1882$, p. 28-36; $i d$., "Épées et poignards de bronze du Morbihan et de la Loire-Inférieure ", dans la Société d'Émulation des Côtes-du-Nord, 1883, p. 126-150. Micault, Victor et LisLE Du Dreneuc, Pitre de, Inventaire des épées et poignards de bronze trouvés dans les cinq départements de la Bretagne, Saint-Brieuc, 1883-1884, 81 p. 
fouillé en 1881, seuls les dessins de Pitre avaient pu permettre à ce monument de contribuer à écrire l'histoire de ce territoire, et de ce monument en particulier.

Figure 50 - Vase à quatre anses du tumulus de Castelow (Sussex, G.-B.), Pitre de Lisle du Dreneuc (cl. musée Dobrée)
Figure 51 - Vase à quatre anses et supports multiples d'un tumulus publié par Édouard Piette, Pitre de Lisle du Dreneuc (musée Dobrée)
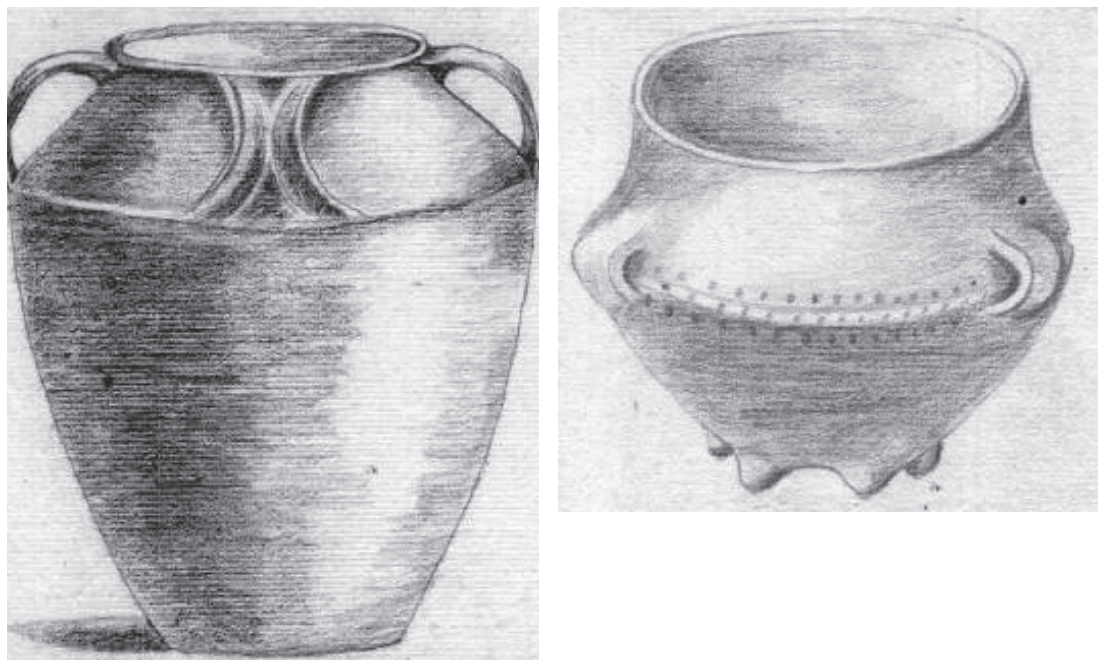

De 1875 à 1892, Pitre et Georges de Lisle ont ainsi exploré une soixantaine de sites préhistoriques (fig. 52). Pitre apparaît comme un archéologue travaillant avec les moyens et les ambitions de son époque, mais il se distingue néanmoins par sa lucidité et le soin apporté à transmettre ses informations aux générations suivantes.

On peut se faire une idée de la méthode de fouilles qu'il a pratiquée avec son frère Georges en relisant certaines de ses publications, et, notamment, dans les descriptions des fouilles du dolmen du Grand Carreau Vert, en $1883^{195}$ (fig. 52), ou du tumulus de La Motte à Sainte-Marie-sur-Mer, en $1892^{196}$. Après autorisation du propriétaire du terrain, il commence par "aplanir le tumulus", c'est-à-dire par le libérer de toute construction, puis il procède à la fouille par "l'ouverture d'une tranchée sur le parcours du chemin " afin de découvrir les

195. LisLE Du DrenEuc, Pitre de, «Fouilles des dolmens du Grand Carreau Vert, Saint-MichelChef-Chef ", Matériaux, 1886, 3, p. 277-285.

196. LisLE Du Dreneuc, Pitre de, « Notice sur la fouille du tumulus de la Motte, Sainte-Marie, Loire-Inférieure ", BSANLI, 31, 1892, p. 199-203. 


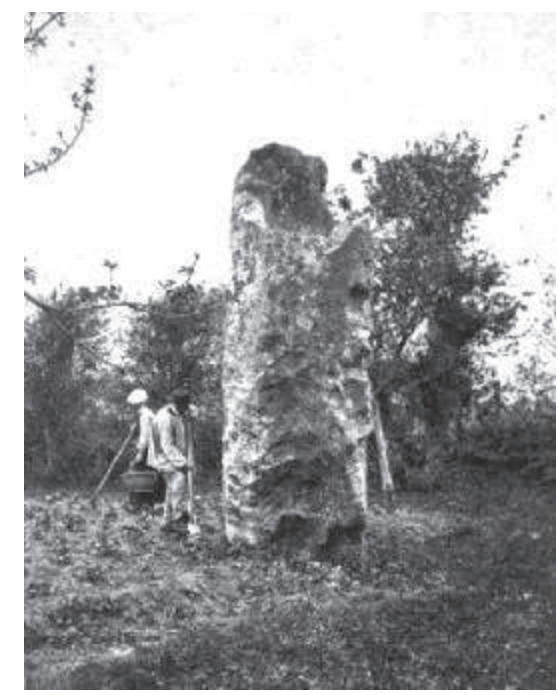

Figure 52 - "Fouilles" de la Pierre Frite, à Basse-Goulaine (L.A.)

(cl. anonyme, 1877, musée Dobrée)

Figure 53 - Dolmens du tumulus du Grand Carreau Vert à Saint-Michel-Chef-Chef (L.-A.), dessin original sur calque, Pitre de Lisle du Dreneuc, 1892 (musée Dobrée)

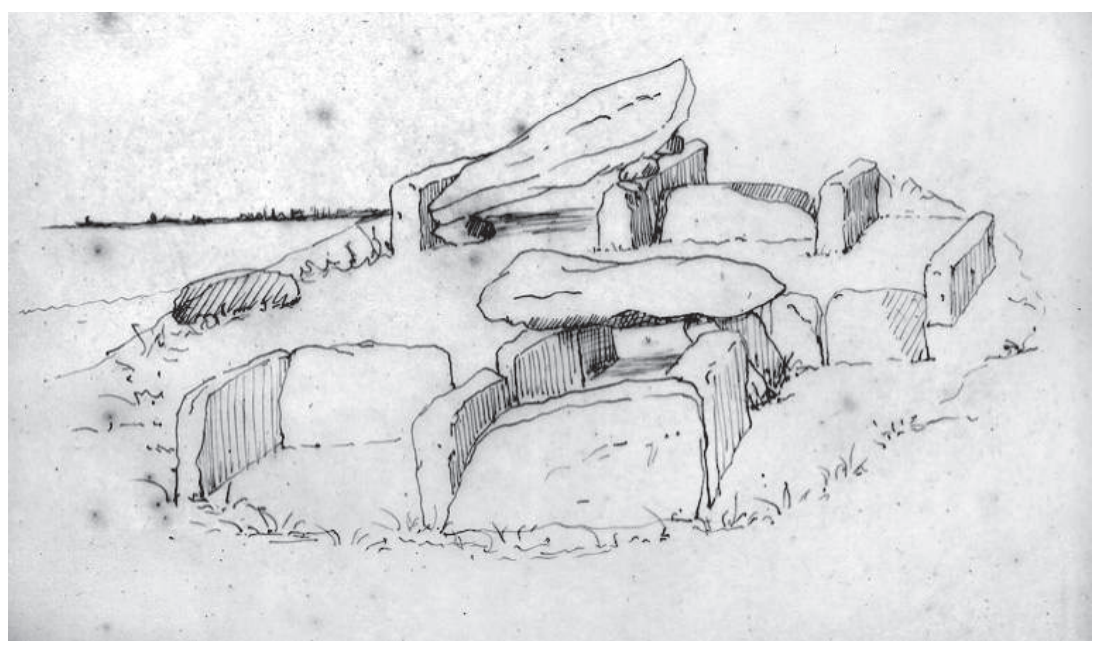

structures (allée couverte), ensuite il fait " enlever le remplissage de moellons qui obstruaient l'entrée " et pénètre dans l'édifice, "enlève les pierres amoncelées à l'intérieur " et peut alors commencer sa fouille. Il repère les couches de sédiments comme cette "terre ocreuse, pulvérulente, ayant cet aspect bien connu qui signale le niveau le plus riche de nos fouilles" ou encore " au-dessous du terrain s'étend un lit d'argile jaune pâle, battue comme l'aire d'une ferme 
bretonne et qui couvre le fond de tout le monument; cette terre est plaquée sur le sol naturel ", et devait être déjà solidifiée quand on a déposé

" les objets funéraires dans le dolmen, car ils étaient placés dessus sans y adhérer. Nous venions à peine d'effleurer le sommet de la terre rouge lorsqu'un coup de pioche fit tout à coup rouler un gros vase [...]. Cette trouvaille nous avertit de nous tenir sur nos gardes, en laissant les piocheurs s'escrimer sur une partie moins avancée du monument, nous continuons seuls la minutieuse besogne du triage des terres [...]. En palpant les terres amassées le long du premier montant qui soutient la table, nous rencontrons la surface ronde et polie d'un énorme vase [...]. Après avoir dégagé à la pointe du couteau les terres qui l'englobaient, nous soulevons avec des précautions maternelles ce doyen de la poterie celtique. [Enfin,] en palpant les terres mêlées de charbon, vers le centre du caveau, je ressentis au doigt une piqûre très vive causée par la pointe acérée d'une flèche. "

Pour résumer la méthode : l'archéologue n'étudie pas la structure extérieure des édifices et se contente de l'intérieur des monuments dans l'espoir d'y trouver des objets. Cette méthode d'exploration des monuments mégalithiques est conforme à la pratique de l'époque : on "visite" les édifices ${ }^{197}$. Si l'exploration extérieure est verticale, en revanche, à l'intérieur de l'édifice le dégagement des sols est accompli selon une méthode horizontale : on distingue les couches de couleur et de nature différentes, mais on ne peut pas encore parler de méthode stratigraphique. Toutefois, l'archéologue est capable de distinguer les différents plans intérieurs des monuments et prend des mesures systématiquement. Le mobilier est recueilli mais le lien n'est pas fait entre le relevé des structures, la position des objets et la stratigraphie pour dégager les caractères principaux du monument et contribuer ainsi à la connaissance du mobilier qu'il renfermait.

Les publications de Pitre sont généralement des comptes-rendus de fouilles rédigés un peu comme l'aventure d'un "chasseur de trésor" :

"C'est toujours un moment de suprême attraction que celui où l'on pénètre pour la première fois dans un dolmen inviolé : les grandes pierres qui se dressent autour de vous comme pour défendre leur trésor, la lueur pâle dessinant à demi les objets ensevelis depuis tant de siècles, tout vous reporte vers un passé dont le mystérieux lointain vous fascine comme les regards du Sphinx ${ }^{198}$."

Pitre de Lisle du Dreneuc a toutefois conscience de ses propres limites et du fait que la recherche en Préhistoire n'en est encore qu'aux premiers

197. Cependant, la démarche de Pitre et Georges de Lisle est beaucoup plus "scientifique" que celle de Gustave Paille, d'abord théoricien de la fouille des mégalithes, puis véritable chasseur d'objets qui n'hésite pas à faire parler la poudre pour déterrer des pièces destinées à constituer le musée privé de son commanditaire, le marquis PierreAuguste de Montaigu, au château de la Bretesche, en Missillac (Loire-Atlantique) : BLAIN, Hugues-François et SANTROT, Jacques, " Gustave Paille, un archéologue "à façon” en Basse Bretagne (1898-1905) ", ABPO, 107, 2000, 3, p. 111-115, et p. 143.

198. Lisle Du Dreneuc, Pitre de, " Notice sur les fouilles du tumulus de La Motte, SainteMarie (Loire-Inférieure) ", BSANLI, 31, 1892, p. 200. 
balbutiements. Dans son Dictionnaire archéologique, en parlant des monuments mégalithiques, et en particulier de ceux de Pornic, il écrit :

"Nous passerons en revue chacun de ces groupes, décrivant avec le plus de soin possible les dimensions et la forme de chaque galerie. Je sais très bien que ces descriptions monotones [...] sont parfaitement ennuyeuses; je le sais d'autant mieux que j'ai pris la peine de les relever et de les transcrire. [...] et voici pourquoi je l'ai fait. Plus tard, nos descendants en sauront plus long que nous, je l'espère bien; ils pourront classer les mégalithes, leur trouver des styles et les répartir en différentes époques, suivant leur mode de construction. Mais, alors, il ne leur restera plus guère de dolmens pour exercer leur savoir. Il est donc bon de conserver par des descriptions exactes, complétées par des plans et des dessins, ces monuments que nous avons encore sous nos yeux et qui, pour la plupart, n'ont que peu de temps à vivre ${ }^{199}$."

Ce sont bien là les caractéristique d'une démarche scientifique, marquée par le respect des faits observés, l'objectivité d leur description, l'humilité du chercheur dans ses interprétations. Pitre de Lisle se considère comme le simple maillon d'une longue chaîne de chercheurs et il encourage ses collègues à comprendre l'importance des relevés qui sont susceptibles de devenir l'unique trace des monuments.

\section{Pitre de Lisle, second conservateur du musée départemental d'Archéologie (1882-1924)}

À la mort de Fortuné Parenteau (10 septembre 1882), la Société archéologique se réunit en séance extraordinaire le 26 septembre 1882 pour nommer Pitre de Lisle du Dreneuc nouveau conservateur du musée départemental d'Archéologie ${ }^{200}$, nomination ratifiée le 5 octobre par arrêté préfectoral $^{201}$. Le nouveau conservateur avait reçu l'appui du préhistorien Émile Cartailhac, directeur de la revue Matériaux pour l'histoire primitive et naturelle de l'homme, et, à la séance du 7 novembre 1882, le baron Héracle Olivier de Wismes fait l'éloge de Pitre de Lisle du Dreneuc "qui succède à M. Parenteau comme conservateur du Musée archéologique de Nantes. Cet honneur revenait de droit à $M$. de Lisle, dont le dévouement et le zèle sont si connus, et confirme l'opinion générale que personne mieux que lui ne pouvait remplir ces délicates fonctions ${ }^{202} "$.

Dès le 10 novembre 1882, le préfet le charge de deux missions prioritaires : dresser l'inventaire des différents mobiliers conservés au musée de l'Ora-

199. LISLE DU DRENEuc, Pitre de, Dictionnaire archéologique, 1882, p. 266.

200. Lisle du Dreneuc, Pitre de, Catalogue du musée archéologique de Nantes (= Cat. 1903), $3^{\mathrm{e}}$ édition, Nantes, imprimerie moderne, Joubin et Beuchet Frères, 1903, p. VII.

201. Archives du musée Dobrée, courrier de remerciement du préfet, Anatole Catusse, à Émile Cartailhac, 10 octobre 1882. Le musée n'ayant pas conservé les archives des Commissions administratives entre 1878 et 1886, on ne sait pas si cette nomination a fait l'objet d'une réunion de la Commission.

202. BSANLI, 21, 1882, p. x, 7 novembre 1882. 
toire ${ }^{203}$, et rédiger le catalogue précis de la collection Parenteau ${ }^{204}$, ce qu'il fera, partiellement seulement, en 1883. Ce document n'a malheureusement pas été retrouvé mais le préfet Catusse en accuse réception ${ }^{205}$. L'année suivante, en même temps qu'il lui réclame un inventaire de la collection de Charles Seidler, le préfet demande au conservateur un exemplaire, imprimé cette fois, de ce catalogue des collections Parenteau car " le document que je possède est incomplet; il serait bien long de le copier ${ }^{206}$ ". On doit certainement aux bonnes relations entretenues par Pitre de Lisle du Dreneuc avec l'Anglais Charles Seidler, l'entrée de l'importante collection de ce dernier au musée départemental de l'Oratoire. De ce personnage, sans doute installé à Nantes pour affaires vers 1860, on ne sait que très peu de choses. Ami de Fortuné Parenteau à qui il a donné accès à sa collection ${ }^{207}$, il est déclaré " savant archéologue " par Pitre de Lisle du Dreneuc ${ }^{208}$.

Devenu membre résidant de la Société archéologique le $1^{\text {er }}$ juillet 1873 en même temps que René de Kerviler ${ }^{209}$, puis titulaire de 1875 à 1883, Seidler y présente en 1879 sa collection préhistorique du Danemark ${ }^{210}$. Membre du comité central de la Société de 1880 à 1883, il décide avant son retour à Londres, de mettre en vente sa collection composée d'archéologie locale, française et européenne, mais aussi d'ethnographie africaine, américaine et océanienne, comme il l'annonce à Pitre de Lisle du Dreneuc ${ }^{211}$. Il

\section{Figure 54 - Portrait de Charles} Seidler, avant 1882 (archives photographiques du musée Dobrée - cl. anonyme, musée Dobrée)

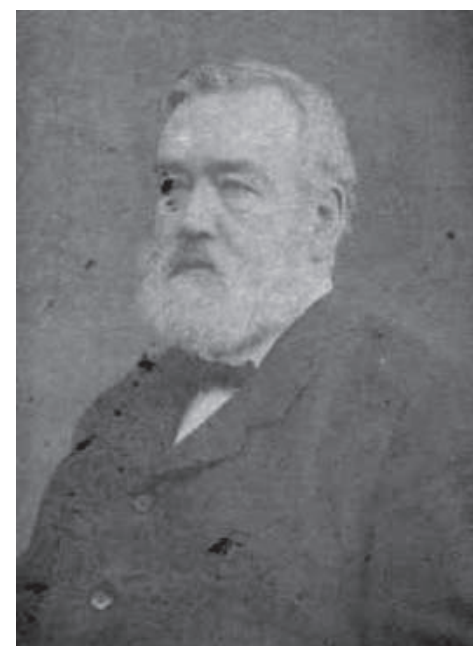

203. Archives du musée Dobrée, lettre du 10 novembre 1882 du préfet Anatole Catusse au nouveau conservateur.

204. Archives du musée Dobrée, lettre du 13 août 1883 du préfet Anatole Catusse à Pitre de Lisle du Dreneuc.

205. Archives du musée Dobrée, lettre du préfet Anatole Catusse, 26 janvier 1884 .

206. Archives du musée Dobrée, lettre du préfet Anatole Catusse, 31 octobre 1884.

207. Fortuné Parenteau a dessiné certains objets de la collection Seidler pour illustrer, à titre de comparaison, son Inventaire archéologique de 1878.

208. LiSLE DU Dreneuc, Pitre de, Cat. 1903, p. 9.

209. BSANLI, 12,1873, p. 101, $1^{\mathrm{er}}$ juillet 1873. Il est présenté à la Société par Alexandre Perthuis et Jean Prosper Hyrvoix.

210. BSANLI, 18, 1879, p. 17, 18 février 1879.

211. Archives du musée Dobrée, lettre de Charles Seidler, 10 août 1883, dans le Catalogue énonciatif du musée archéologique, 1868-1902, n 1360. Charles Seidler précise qu'il a fait " un inventaire de sa collection qui comprend 1300 pièces et [...] [qu'il] désire vivement qu'elle reste à Nantes". Dans ce même document, Pitre de Lisle explique "je connaissais depuis des années les belles séries d'armes anciennes ou étrangères recueillies par le savant anglais et leur aliénation me semblait un véritable malheur pour nos études. Le chiffre de $17000 \mathrm{~F}$ 
a toujours entretenu d'excellentes relations avec le musée départemental d'Archéologie (il est devenu membre de sa Commission administrative en 1882), et avec Nantes « où il comptait vingt années d'hospitalité affectueuse ${ }^{212}$ ». Après bien des péripéties, cette collection est achetée par la Ville de Nantes le 4 mars $1884^{213}$. C'est l'occasion pour la Société archéologique de ranimer de vieilles querelles avec le Département, comme le soulignent les membres de la Société archéologique dans un courrier de remerciement à la Ville de Nantes :

" Ils [les membres de la Société archéologique de la Loire-Inférieure] s'empressent d'applaudir au vote du Conseil municipal de la Ville de Nantes qui a permis à la Mairie d'acquérir la riche collection d'objets préhistoriques et curieux laissée par M. Seidler; ils se réjouissent de penser que le Musée de Nantes sera bientôt le plus beau de la province, ils remercient très sincèrement messieurs les conseillers [municipaux] de la faveur avec laquelle ils accueillent les propositions qui ont pour but le développement de la science des Antiquités Nationales; ils émettent, à l'exemple du Conseil municipal, le vœu que le musée archéologique, si bien patronné par la Ville, devienne un établissement municipal et prient Monsieur le Maire d'entrer en négociations avec l'Administration départementale pour obtenir la cession le plus tôt possible ${ }^{214}$."

Il faut reconnaître que la situation est un peu ambigüe car Jules de La Laurencie, qui vient de quitter la présidence de la Société archéologique en janvier 1884, est aussi conseiller municipal.

Huit ans plus tard, à la demande du conservateur, la municipalité cède la collection Seidler au Département de la Loire-Inférieure ${ }^{215}$; en attendant, en 1886, elle accorde au musée une subvention exceptionnelle de $1000 \mathrm{~F}^{216}$

demandé par M. Seidler était relativement faible, mais avec notre budget de $1000 \mathrm{~F}$ par an, il ne fallait pas songer à l'aborder" .

212. L'Espérance du Peuple, dimanche 9 mars 1884, achat de la collection Seidler.

213. LISLE Du DRENEuc, Pitre de, " La collection Ch. Seidler au musée archéologique de Nantes ", BSANLI, 23, 1884, p. 68-70.

214. Archives du musée Dobrée, autographe 2401, dans Catalogue des lettres, chartes et pièces manuscrites (1 à 2551), Collection Thomas Dobrée et collection du musée archéologique, Nantes, imprimerie moderne, 1906, p. 979, lettre non datée (mars 1883?), signée, notamment, par Jules de La Laurencie, Héracle Olivier de Wismes, Eugène Orieux, Émile Grimaud, Arthur des Jamonières, le marquis de Granges de Surgères, Paul du Chatellier, Charles Perrion, Alexandre Perthuis, Constant Verger, Régis de l'Estourbeillon, Léon Maître, Paul Soullard, Auguste Laurant, Joseph Rousse, Gustave de La Brosse, Auguste Bacqua, François Bougouin, le docteur Porson, Louis Viau, le docteur C. Merland, Alexandre de Monti de Rezé, Charles Riardant, Xavier de La Touche, Henri de La Peyrade, le docteur Genuit, Jules du Champ Renou et Jules Montfort. On remarquera que le conservateur du musée, Pitre de Lisle, n'a pas signé ce courrier.

215. En septembre 1886, le conservateur demande encore l'autorisation à la Ville de Nantes de lui prêter quelques pièces de la collection Seidler pour une exposition à l'occasion de la tenue à Nantes du congrès de la Société française d'Archéologie : archives du musée Dobrée, lettre d'Édouard Normand, maire de Nantes, au conservateur (16 septembre 1886) ; compterendu de la réunion de la Commission administrative du 17 février 1892, avec mentions marginales : "La collection Seidler appartient à la Ville " et « M. le maire a répondu affirmativement à la demande verbale de transposition de la collection présentée par le conservateur ".

216. Archives du musée Dobrée, courrier du maire de Nantes, Alfred Riom, au président de la Commission administrative du musée archéologique, 23 décembre 1892. La municipalité refuse en 1892 une nouvelle demande de subvention exceptionnelle. 
" pour subvenir aux frais de surveillance, d'entretien, d'augmentation même des collections urbaines, comme aussi pour couvrir les dépenses résultants de leurs manipulations éventuelles provoquées par les exigences du local de l'Oratoire 217 ". Le Conseil général s'engage, en échange du don de la collection Seidler, à en assurer la présentation ${ }^{218}$ mais la Ville de Nantes continue à prendre en charge l'assurance des collections Parenteau et Seidler contre l'incendie ${ }^{219}$. Il y a donc, dans les faits, quelque confusion entre le caractère départemental et municipal de ce musée dont les moyens de fonctionnement ne sont guère brillants : en 1886, la Commission administrative du musée reçoit du conservateur un rapport alarmiste :

"La situation financière du Musée est déplorable [le budget annuel est alors de $1000 \mathrm{~F}$ ]. Un musée ne peut réellement vivre s'il n'a pas les fonds nécessaires pour exposer au public des collections convenablement installées [...] et surtout $s$ 'il ne peut acquérir les pièces les plus indispensables pour l'étude. Pour cela, il lui faut un budget, et le musée archéologique n'en a pour ainsi dire pas ${ }^{220}$."

Le rapport établit un comparatif avec les budgets des autres musées archéologiques des grandes villes de France : “Amiens, $20000 \mathrm{~F}$, Lille $42700 \mathrm{~F}$, Lyon, $63000 F$ ", et il ajoute, en comparaison, les budgets des musées de la Ville de Nantes : "Le musée des Beaux-Arts reçoit annuellement $7150 \mathrm{~F}$, celui d'Histoire naturelle $7100 \mathrm{~F}$. "Cette requête demeure sans réponse.

Dans ces années 1880, en raison de l'état des finances du musée, encore installé à l'Oratoire, les achats se font très rares et l'enrichissement provient essentiellement de dons : collections locales de Sylvain-Alcide Bord et de René de Kerviler après les fouilles du bassin de Penhoët, à Saint-Nazaire, par exemple ${ }^{221}$, "qui remplissent à elles seules tout un côté de la nef de l'Oratoire $^{222}$ ", de la cathédrale de Nantes ${ }^{223}$, de la Porte Saint-Pierre ${ }^{224}$, de Petit-

217. BSANLI, 25, 1886, p. xxvı, 9 février 1886. La municipalité charge la Société archéologique de la gestion de cette subvention.

218. Archives du musée Dobrée, minute d'une délibération du Conseil municipal (s.d.) : "Le Département a fait exécuter à son compte les vitrines destinées à la collection Seidler (20 juin 1885) pour 2293,20 F. Les soubassements, chêne et glace, ajoutés postérieurement, ont fait monter l'ensemble de la dépense à 4489,94 F."

219. Archives du musée Dobrée, lettre du préfet Anatole Catusse au conservateur, 31 octobre 1884.

220. Archives du musée Dobrée, Commission administrative, séance du 15 juillet 1886.

221. Archives du musée Dobrée, Catalogue énonciatif 1869-1902, n 1350 et 1351, et séance de la Société du $1^{\text {er }}$ avril 1884, BSANLI, 23, 1884, p. xLIV et XLV.

222. "Musée départemental d'Archéologie ", La Ville de Nantes et la Loire-Inférieure, Nantes, 1898, II, p. 77.

223. C'est grâce au maire de Nantes, Georges-Évariste Colombel, que le produit des fouilles de la cathédrale, financées par la Ville de Nantes, entre au musée départemental d'Archéologie : archives du musée Dobrée, courrier de Colombel au conservateur, 21 août 1884, l'informant du transport des objets restés "dans la salle privative de la Société archéologique".

224. LEGENDRE, Alfred, Nantes à l'époque gallo-romaine d'après les découvertes faites à la Porte Saint-Pierre, Nantes, C. Mellinet, 1891. 
Mars, de Mauves-sur-Loire ${ }^{225}$ ou de Fégréac (Loire-Atlantique) ${ }^{226}$. Le musée reçoit également les collections brésiliennes (Bas-Amazone) de l'abbé Auguste Cullère en huit envois successifs, de 1884 à 1894 (309 pièces). Ami de l'abbé Cullère avec lequel il entretient une importante correspondance ${ }^{227}$, et avec l'aide du docteur Hamy, directeur du musée d'Ethnographie du Trocadéro, Pitre de Lisle du Dreneuc se passionne pour ces découvertes lointaines et publie quelques pièces de la collection (fig. 55) ${ }^{228}$.

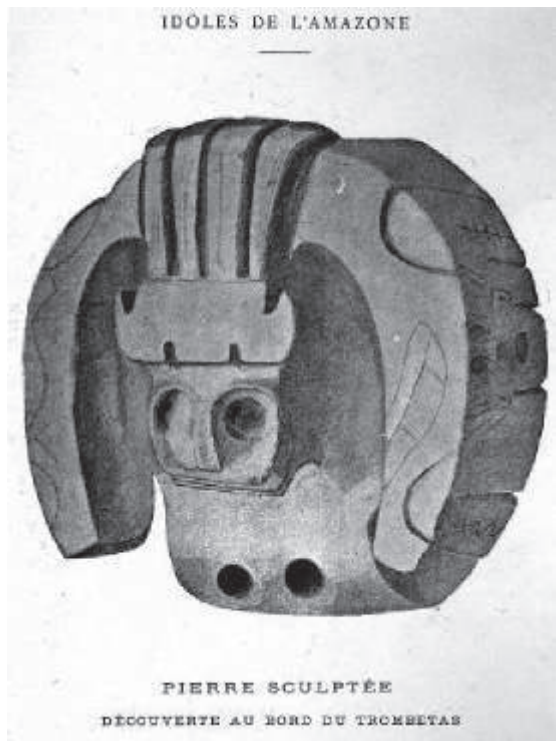

Figure 55 - Idoles de l'Amazone, pierre sculptée au bord du Trombetas : amulette amazonienne « représentant un mauvais génie » (Pitre de Lisle du Dreneuc, Nouvelles découvertes d'idoles de l'Amazone, Paris, 1889, musée Dobrée)

Découragé par l'encombrement chronique et sans issue de la chapelle de l'Oratoire, Pitre de Lisle trouve une nouvelle fois un secours auprès de la Ville de Nantes qui met à sa disposition, en mars 1885, le rez-de-chaussée de l'ancienne caserne de la Visitation ${ }^{229}$. L'année suivante, pour faire de la place

225. BSANLI, 23, 1884, p. LXV, 2 décembre 1884. MAÎTRE, Léon, « La station gallo-romaine de Vieille-Cour à Mauves ", BSANLI, 24, 1885, p. 101-107, suivi des " Monuments romains de Coussol et du Breil en Petit-Mars (Loire-Inférieure) ", BSANLI, 25, 1886 (1), p. 91-114; " La station gallo-romaine de Mauves ", BSANLI, 25, 1886, 2, p. 26-48. Fouilles de Léon Maître, archiviste départemental.

226. MAîTRE, Léon, " La station gallo-romaine de Rieux-Fégréac ", BSANLI, 26, 1887, 2 , p. 1-34.

227. Archives diplomatiques de Nantes, dossier Légation de France à Rio, affaires religieuses, série A, 2 MI13170, 166/3 et fonds Archives des Postes, série A 126, 184/4, 207/5, 75 ; archives du musée Dobrée, fonds "Donateurs", s.v. Auguste Cullère.

228. Lisle du Dreneuc, Pitre de, Nouvelles découvertes d'idoles de l'Amazone, Paris, Émile Lechevalier, 1889.

229. Archives du musée Dobrée, lettre du maire de Nantes, Édouard Normand, au conservateur, 18 mars 1885. 
et éliminer un certain nombre d'objets, la Commission départementale autorise le conservateur à pratiquer des échanges avec les collections du musée départemental d'Archéologie ${ }^{230}$. Cette même année, à la demande de la Ville de Nantes, il fait partie du comité d'organisation d'une exposition consacrée à l'Art ancien et à l'archéologie organisée à l'occasion du Congrès de la Société française d'Archéologie à Nantes ${ }^{231}$. La Société d'archéologie adhère à ce projet estimant " qu'une telle exposition est le complément nécessaire du Congrès et une occasion unique pour les Nantais eux-mêmes d'admirer les richesses toujours cachées que renferment les collections particulières de nos compatriotes. M. Léon Maître estime que les cloîtres de la Visitation pourraient être parfaitement appropriés à cet objet ${ }^{232}$ ». Outre une partie de la collection Seidler et les pièces maîtresses du musée départemental d'Archéologie, Pitre propose de présenter trois œuvres majeures de Nantes : le "reliquaire" du cœuer d'Anne de Bretagne (conservé à la mairie), le Livre d'heures d'Anne de Bretagne et le tome I de la Cité de Dieu (conservés à la Bibliothèque publique) ${ }^{233}$. Ses publications scientifiques et le succès de l'exposition lui vaudront de recevoir la grande médaille de vermeil du Congrès de la Société française d'Archéologie.

En 1887, c'est à son tour de faire le don, avec son frère Georges, d'une partie de leurs collections paléolithiques provenant de leurs fouilles locales de 1883 ("stations" du Pas-Chalène et de L'Ouchette à Montbert, du Breil et de Bégrolle à La Haye-Fouassière, Loire-Atlantique) et autres : outillage lithique et osseux de Laugerie-Haute, aux Eysies-de-Tayac, et de La Madeleine, à Tursac (Dordogne) ${ }^{234}$, du Bois du Rocher, à Saint-Hélen (Côtes-d'Armor). 289 objets entrent ainsi dans les collections du musée ${ }^{235}$.

En 1888, la situation du musée empirant, le conservateur écrit à Jules de la Laurencie, conseiller municipal et président honoraire de la Société archéologique en dressant un état de la situation et en précisant les besoins, tant techniques que scientifiques :

230. Archives du musée Dobrée, relations du musée avec la préfecture, lettre du préfet Paul Glaize au conservateur, 3 avril 1886 : "La Commission départementale vous a autorisé, conformément à votre demande du 29 mars dernier, à échanger des pièces de peu de valeur et qui font double emploi au Musée départemental d'archéologie contre des objets pouvant compléter les collections dudit Musée."

231. Archives du musée Dobrée, courriers du maire de Nantes, Édouard Normand, au conservateur du musée, 25 juin, 12 août, 16 septembre, 2 et 6 octobre 1886 .

232. BSANLI, 24, 1885, p. XV, 3 novembre 1885.

233. Cette " Exposition des Beaux-Arts, d'Archéologie et de Photographie " fit l'objet d'un article, $B S A N L I, 25,1886$, p. 104-107. Dans le discours d'inauguration prononcé le 10 octobre 1886, Anatole de Brémond d'Ars, président de la Société archéologique, énumère les différents prêteurs et fait, pour la première fois, référence à la collection privée du conservateur : "Les antiquités primitives provenant du Musée départemental enrichi des collections Seidler et Kerviler, et que $M$. de Lisle a disposées avec cette méthode parfaite appliquée depuis longtemps à sa collection particulière composée elle-même d'objets essentiellement bretons. "

234. " Le désir d'augmenter ses connaissances l'avait précédemment emmené vers le paradis des préhistoriens qu'est la vallée de la Vézère " : notice nécrologique Hommage au vicomte Pitre de Lisle du Dreneuc, anonyme, sans doute 1924 (archives privées).

235. On ne sait sans doute pas alors que les plus belles pièces des sites fouillés ont été conservées par Pitre de Lisle dans sa collection personnelle. 
"Toutes les richesses accumulées depuis des années et accrues ces temps derniers par les belles collections de MM. Parenteau, Kerviler, Seidler, Bord et St-Léger, forment un fonds important dont il est urgent de tirer parti, car un musée n'existe réellement que lorsque ses collections sont convenablement installées et présentées au public avec ordre et méthode [...]. L'organisation intérieure du musée est donc indispensable, mais pour atteindre ce but, il faudrait pouvoir disposer des ressources suffisantes pour les travaux suivants : $1^{\circ}$ Compléter l'arrangement des collections dans un ordre méthodique [...]. $2^{\circ}$ Préparer les vitrines pour recevoir les objets [...]. $3^{\circ}$ Publier un catalogue et étiqueter les objets [...]. $4^{\circ}$ Enfin, pour arriver à un classement méthodique, il est indispensable de combler les grandes lacunes qui interrompent la série chronologique. Pour qu'un musée de ce genre ait une réelle valeur scientifique et présente un enseignement facile pour tous, il faut que les principales époques y soient représentées, au moins par les pièces les plus caractéristiques $[\ldots]^{236}$.»

Face à chaque rubrique, Pitre de Lisle donne une estimation financière. Il ajoute :

"Ce manque de ressources déterminées est extrêmement préjudiciable aux intérêts du Musée. Il est impossible de donner à l'organisation une marche régulière lorsqu'on est toujours sous le coup d'un vote plus ou moins favorable $[\ldots]$."

Ce courrier est révélateur du caractère "professionnel " la démarche du conservateur et de l'intérêt qu'il porte à " son " musée. Il sera d'ailleurs partiellement entendu car le budget annuel du musée passera de 1000 à 3500 F. Pour sa part, la Ville accordera au musée en 1890 un nouveau budget spécifique $(650 \mathrm{~F})$ pour la confection de vitrines ${ }^{237}$.

À partir de 1890, devenu vice-président de la Société archéologique et correspondant du ministère de l'Instruction publique et des Beaux-Arts pour les travaux historiques (sous-commission des monuments mégalithiques) ${ }^{238}$, Pitre de Lisle du Dreneuc n'a plus le temps de poursuivre des recherches de terrain ${ }^{239}$. À la demande de la Commission administrative du musée, il travaille sur un nouveau règlement intérieur de l'établissement dont on possède plusieurs versions manuscrites avant l'imprimé de $1894^{240}$. Dès la réunion de la Commission administrative du 22 mai 1891, le conservateur donne lecture d'un texte du règlement ${ }^{241}$, " approuvé par la préfecture ", qui comporte quatre articles portant sur les rôles respectifs de la Commission administrative, du trésorier, du conservateur et du gardien du musée. Si la

236. Archives du musée Dobrée, collectionneurs, s.v. Pitre de Lisle du Dreneuc, $\mathrm{n}^{\circ} 31$.

237. Archives du musée Dobrée, courrier du maire de Nantes, Ernest Guibourg de Luzinais, 26 février 1890. Pitre de Lisle ajoute de sa main sur cette lettre : "Musée archéologique, confection de vitrines pour les collections qui y sont dispersées : 646,40 F."

238. Archives du musée Dobrée, courrier de Gabriel de Mortillet, président de la Souscommission des Monuments mégalithiques, du 24 octobre 1895, à Pitre de Lisle du Dreneuc. 239. Lisle du Dreneuc, Pitre de, publiera encore une " Notice sur les fouilles du tumulus de La Motte, Sainte-Marie (Loire-Inférieure) ", BSANLI, 31, 1892, p. 199-203.

240. Règlement du musée départemental, Nantes, impr. Émile Grimaud, 1894, 4 p.

241. Archives du musée Dobrée, Règlements; texte non daté, signé par Charles Marionneau et Paul Eudel. 
Commission administrative a un regard sur les enrichissements, son rôle essentiel porte sur la vérification de la gestion des comptes. Le trésorier gère la subvention départementale, portée à $3500 \mathrm{~F}$ en 1886 et répartie entre les acquisitions $(700 \mathrm{~F})$, les fouilles et les frais de déplacements $(500 \mathrm{~F})$, l'entretien et le chauffage du bâtiment (400 F), les gages du concierge (400 F) et, enfin, le traitement de $1500 \mathrm{~F}$ désormais attribué au conservateur qui n'est plus bénévole. Le concierge assure l'entretien et la sécurité des bâtiments et l'ouverture au public deux jours par semaine, les jeudis et dimanches, entre 12 et 14 heures, ainsi que les jours fériés. Il s'occupe également de l'accueil des chercheurs ou des étrangers, sur rendez-vous seulement, avec une interdiction de les laisser photographier ou de prendre des moulages des objets. Enfin, au conservateur, est réservé le rôle scientifique : la classification des collections, leur enrichissement, la tenue de l'inventaire et la rédaction des catalogues. Il est le seul à posséder les clefs des vitrines, " sauf si le président de la commission souhaite en avoir un double".

La Commission administrative du musée départemental d'Archéologie est désormais composée ainsi : sept membres y figurent ès qualité : le préfet de la Loire-Inférieure (Georges Prosper Cleiftie), en tant que président de la Commission, le secrétaire général de la préfecture, vice-président, le maire de Nantes (Ernest Guibourg de Luzinais), l'évêque de Nantes (Jules François Le Coq), le président de la Société archéologique (Anatole de Brémond d'Ars), l'architecte du Département et le conservateur; dix autres membres sont nommés par arrêté préfectoral : Édouard de Kersabiec, ancien conseiller de préfecture, Paul Nau, architecte, Henri de Cornulier, conseiller général, Thomas Dobrée, " numismatiste ", Charles Lechat, ancien maire de Nantes, André Perthuis-Laurant, trésorier du musée, Joseph Rousse, ancien conseiller général et banquier, Paul Eudel, "archéologue et architecte ", Eugène Orieux, agent voyer en chef honoraire du Département, Eugène Boismen, architecte diocésain qui a participé à la construction du palais Dobrée. Ce règlement ne sera totalement publié et appliqué qu'en $1894^{242}$.

Depuis la nomination de Pitre de Lisle au poste de conservateur du musée, les collections passent de 1324 numéros à plus de $5200^{243}$. Cet accroissement a été si rapide, explique le conservateur, que "le local de l'Oratoire est devenu insuffisant. Dès 1891, le Conseil général, dans sa session d'auril, tentait d'acquérir le terrain compris entre le transept sud et le chour; mais, après de longs pourparlers, les négociations échouèrent et l'accroissement du Musée, par suite des nouvelles constructions, devint irréalisable". Le second grand chantier demandé au conservateur par la Commission administrative $^{244}$, est le rangement et le classement des collections dans la

242. Archives du musée Dobrée, Commission administrative 1876-1933, séance du 15 décembre 1894 et approbation en préfecture de 26 décembre 1894.

243. Archives du musée Dobrée, Registre énonciatif 1869-1903, manuscrit, et Cat. 1903, p. VII.

244. Archives du musée Dobrée, Commission administrative 1876-1933, séance du 17 février 1892 
chapelle de l'Oratoire. Les collections sont réparties entre le transept nord, consacré aux antiquités du Vieux Nantes, et le transept sud, consacré aux collections étrangères et à l'ethnographie. Pitre de Lisle souhaite intégrer les antiquités locales de la collection Seidler à la suite des antiquités correspondantes dans lesquelles la collection Parenteau a déjà été intégrée. Il s'agit bien, dans un souci didactique, de rechercher une cohérence chronothématique au détriment de la présentation par collection. Il souhaiterait également que la Commission se prononce sur le fait que "le Musée n'est pas spécialement destiné aux seules collections d'objets trouvés dans le département ", ce que la Commission accepte : "Le Musée est départemental mais ce titre n'explique nullement qu'il soit astreint à contenir uniquement les objets provenant de la Loire-Inférieure ${ }^{245}$. " Pitre de Lisle propose alors un changement de nom du musée qui de Musée départemental d'Archéologie devient Musée Archéologique de Nantes et de la Loire-Inférieure ${ }^{246}$. Pour l'inventaire et la numérotation des pièces, Pitre de Lisle pense qu'il est nécessaire, "en vue de la publication du catalogue, de classer les objets par vitrines, procédé adopté maintenant dans tous les grands Musées et qui a l'avantage de permettre une mise en place immédiate des objets nouvellement venus". Enfin, dernier point abordé, le local de la Visitation mis à la disposition du musée par la Ville en 1885 n'est pas surveillé et les objets y sont entassés, ce qui pose de graves problèmes de sécurité et de conservation des collections. C'est pourquoi le conservateur y a procédé à un tri draconien des objets ${ }^{247}$ : "Il a fallu faire disparaître un nombre prodigieux de tuiles, de carreaux, de briques à rebords, de fragments de poteries et de bétons qui nous arrivaient de tous côtés ${ }^{248}$. "Ce tri des collections est poursuivi en dans la chapelle de l'Oratoire 1894 et $1895^{249}$. Le conservateur est autorisé à ne

245. Malgré le vote par l'Assemblée départementale, unanime, le 22 juin 2009 , d'un nouveau Projet scientifique et culturel du musée Dobrée tenant compte du périmètre des collections du musée, qui résultent de son histoire et ont fait sa réputation, certains se posent encore la même question.

246. Archives du musée Dobrée, Commission administrative 1876-1933, séance du 9 mars 1894.

247. Il n'est d'ailleurs pas le seul et il précise que ces prédécesseurs en ont fait autant avant lui et qu'il s'agit d'objets " sans provenance indiquée et qui sont encore entassés dans des sacs ou dans des tiroirs, mêlés à des pièces modernes de tous les pays ".

248. Archives du musée Dobrée, Commission administrative 1876-1933, séance du 17 février 1892 : "Dans les premières années de la fondation du Musée, on a cherché à remplir rapidement le vaste local de l'Oratoire. Beaucoup de débris sans valeur ont dû être éliminés depuis, et disposés dans un local que la Ville a mis à notre disposition, mais où rien n'est surveillé."

249. Archives du musée Dobrée, Commission administrative 1876-1933, séances du 20 juin 1894 et du 18 mai 1895 : le conservateur est autorisé à porter sur 469 numéros du registre d'entrées la mention "Annulé ". Ces pièces semblent provenir avant tout de la réserve. Georges Durville, dans l'introduction de son Catalogue du musée lapidaire, écrit en 1927, que certaines pièces qui ne portent pas la mention "Annulé " semblent avoir " complètement disparu, sans qu'on puisse dire comment. Nous soupçonnons qu'elles ont dû être victimes des divers déménagements du Musée lapidaire. Quand il était à la chapelle de l'Oratoire, on avait transporté, sous les cloîtres de la Visitation, un certain nombre de pierres que cette chapelle ne pouvait contenir. Rien d'étonnant à ce que quelques-unes de ces 
pas inscrire sur l'inventaire tous les objets qui entrent au musée car " peu à peu les trois conservateurs qui se sont succédés ont diminués bon nombre d'objets encombrants ". Désormais, deux inventaires seront produits : l'un pour les collections conservées, l'autre pour les collections écartées; ils seront régulièrement validés par la Commission administrative du musée. Si la Commission accepte certains procédés de mise en sécurité des œuvres proposés par le conservateur, en revanche, le procédé proposé par Pitre, pour protéger le "reliquaire" du cœur d'Anne de Bretagne est refusé par la Commission car " présentant un danger trop sérieux pour ceux qui seraient appelés à toucher cet objet sans être prévenus des précautions à prendre ${ }^{250}$ ". Pour cet important travail de classement des collections et, surtout, pour l'article que le conservateur consacre aux origines de Nantes ${ }^{251}$, la Société archéologique l'honore de sa première médaille d'or du concours triennal créé en 1892, pour la Société, par le marquis Albert de Dion ${ }^{252}$.

Après la grande opération de tri, les enrichissements sont de nouveau possibles et l'entrée de deux ensembles importants agitent la Commission administrative en $1892^{253}$ : l'achat, pour $1500 \mathrm{~F}$, de la collection de l'abbé Prigent (mobilier du Bronze ancien et de l'âge du Fer des tumulus de Tossen Kergourognon en Prat-Trémel, Côtes-d'Armor), et le don de la collection des jetons des maires de Nantes et des États de Bretagne d'Alexandre PerthuisLaurant, trésorier de la Commission administrative du " musée d'archéologie et d'ethnographie ". Si la première fait l'objet de nombreux débats sur le prix et l'état des finances du musée, - elle sera finalement divisée en trois lots et le musée n'en achètera qu'une partie -, la seconde présente, en contrepartie du don, l'obligation faite par le donateur de publier la collection dans le nouveau catalogue du musée, ce qui sera fait en 1903. À l'occasion de ce don, la Société archéologique fait réaliser une vitrine spécifique pour installer la collection Perthuis-Laurant ${ }^{254}$.

En 1894 et 1895, la donation de sa "propriété des Irlandais " par Thomas Dobrée et le legs de "ses collections de tableaux, gravures, livres, œuvres d'art, monnaies, manuscrits et autographes " au Département "à charge pour lui d'y

pièces aient été égarées, perdues, ou même brisées, lors de leur dernier transfert ". Parmi les pièces disparues, il mentionne une stèle trouvée en 1887 sous la Porte Saint-Pierre, des sarcophages provenant de Rezé, quelques pierres trouvées au Bouffay en 1850.

250. Ce dispositif n'est malheureusement pas expliqué dans le procès-verbal de la réunion de la Commission administrative du 17 février 1892.

251. LISLE DU DRENEUC, Pitre de, « Recherches archéologiques sur les origines de Nantes, première partie, Corbilon ", BSANLI, 32, 1892, p. 183-198, et tiré à part publié à Vannes en 1892. 252. BSANLI, 32, 1892, p. 213-214, 5 décembre 1892. Ce prix a été créé pour récompenser les meilleurs travaux sur Nantes ou sur l'ancien comté nantais. On y apprend que Pitre de Lisle était en concurrence avec le marquis Georges Balby de Vernon pour son article sur l'église de Saint-Julien-de-Vouvantes.

253. Archives du musée Dobrée, Commission administrative 1876-1933, 17 février et 3 octobre 1892, 9 mars 1894.

254. " Discours de M. de la Nicollière-Teijeiro ", nouveau président de la Société, BSANLI, 32, 1893, p. 62, 7 mars 1893. 
entreposer les collections dudit sieur Dobrée 255 ", arrive à point nommée pour le musée départemental d'Archéologie, et inversement, car le Département n'eut sans doute pas accueilli la donation Dobrée s'il n'avait préalablement possédé le musée départemental d'Archéologie. D'après Georges Durville, Pitre de Lisle a joué un rôle essentiel dans la décision de Thomas Dobrée de céder ses bâtiments et ses collections au Département de la Loire-Inférieure :

"Avec son caractère hésitant, il [Thomas Dobrée] ne savait trop à quel parti s'arrêter pour la conservation de ses collections dans le palais qu'il leur avait destiné. M. P. de Lisle finit par le décider à léguer au Département, meubles et immeubles, toutes les richesses dont l'avenir l'inquiétait ${ }^{256}$."

Dès la séance de la Commission administrative du 27 septembre 1894, Pitre de Lisle soulève le problème du déménagement des collections et du " monument Dobrée "; il pense que le "manoir de Jean V fait un asile sérieux pour nos collections qui trouveront à s'établir royalement dans leur nouveau local $^{257}$ ". Toutefois Thomas Dobrée a mis certaines conditions à sa donation :

"Le Département pourra, si cela lui convient, installer ses collections archéologiques dans les bâtiments annexes séparés de l'édifice principal affecté au musée, et, notamment, dans le manoir de Jean v, mais à la condition, dans ce cas, d'isoler la partie de l'immeuble consacrée aux collections archéologiques départementales, et de lui ménager une entrée spéciale, de façon que les personnes venant au Musée archéologique n'aient pas accès, par ce fait, dans le reste de la propriété et, notamment, au Musée Dobrée ${ }^{258}$."

Le 31 mars 1895, dans un codicille, Thomas Dobrée désigne Pitre de Lisle du Dreneuc comme conservateur du futur "musée Thomas Dobrée ${ }^{259}$ ". Lors de sa première réunion, le $1^{\mathrm{er}}$ avril 1896, la nouvelle Commission administrative du musée Thomas Dobrée entérine cette nomination ${ }^{260}$.

En 1895 encore, avant le déménagement du musée départemental d'Archéologie, sa Commission administrative renouvelée ${ }^{261}$ autorise de nouveau le conservateur à procéder à l'élimination de nombreux objets :

255. Lisle Du Dreneuc, Pitre de, "Musée Th. Dobrée ", La Ville de Nantes et la LoireInférieure, II, Nantes, 1898, p. 44-66.

256. L'Écho de la Loire, février 1924, nécrologie de Pitre de Lisle du Dreneuc.

257. Archives du musée Dobrée, Commission administrative 1876-1933, séance du 27 septembre 1894.

258. Archives du musée Dobrée, Extrait de la donation de Thomas Dobrée du 8 août 1894, article 5, repris dans APTEL, Claire, BIotTEAU, Nathalie, RichARD, Marie et SANTROT, Jacques, Thomas Dobrée, 1810-1895, un homme, un musée, Nantes, musée Dobrée, et Paris, Somogy, cat. d'expo., 1997, p. 317, art. 5.

259. Codicille de Thomas Dobrée, 31 mars 1895, dans lequel le testateur nomme les membres de la Commission administrative qu'il met en place et désigne le conservateur du musée Thomas Dobrée : "Pitre de L'Isle du Dréneuc que je nomme Conservateur de mon Musée ", Archives municipales de Nantes ( $8^{\circ} \mathrm{Z} 2 \mathrm{~A} 161$ ), repris par SANTROT, Jacques dans APTEL et al., op. cit., p. 318.

260. Archives du musée Dobrée, Commission administrative du musée Thomas Dobrée, $1^{\mathrm{er}}$ avril 1896.

261. Archives du musée Dobrée, Commission administrative 1876-1933, 15 décembre 1894. 
" Il y a un inconvénient sérieux à garder cette agglomération de débris qui forcément empêchera l'ordre d'être parfait [...]. En somme, le principe à admettre nous semble devoir être celui que suit tout bon collectionneur : exposer seulement les pièces choisies, garder en caisse ou dans des tiroirs les fragments pouvant servir pour l'étude, et éliminer franchement le fatras ${ }^{262}$."

Le conservateur doit annuler les objets concernés sur le registre d'entrée.

\section{Conservateur du musée départemental d'Archéologie et du musée Thomas Dobrée (1896-1924)}

Le conservateur est chargé du transfert des collections archéologiques $^{263}$ de la chapelle de l'Oratoire dans "l'immeuble départemental de la rue des Irlandais ${ }^{264}$ ".

En 1896, les collections de Thomas Dobrée sont encore dans le logement qu'il occupait jusqu'à son décès, le 3 octobre 1895 , au 4 étage du 1 place Graslin, à l'angle de la rue Crébillon ${ }^{265}$. Dans un courrier adressé au préfet Cleiftie, "au nom de la Commission administrative du Musée Dobrée " présidée par Eugène Orieux, Pitre de Lisle demande leur transfert au $1^{\text {er }}$ étage "des Irlandais" (c'est-à-dire du palais Dobrée) ${ }^{266}$. Toutefois ce transfert ne peut se faire qu'après l'achèvement de l'escalier d'honneur et du cloisonnement, la création de cheminées et d'un calorifère, la construction de vitrines, etc. Certaines sculptures demeureront inachevées. En outre, les collections du donateur étant insuffisantes pour occuper tout le palais, le conservateur propose à la Commission de présenter provisoirement les collections archéologiques au rez-de-chaussée, et les collections Dobrée, au $1^{\mathrm{er}}$ étage du palais. Les sommes nécessaires aux aménagements et au transfert des collections Dobrée sont prises sur la donation de Thomas Dobrée et réglées par son exécuteur testamentaire, Hippolyte Durand-Gasselin ${ }^{267}$. Georges Durville explique les difficultés de son prédécesseur :

" Il fallait adapter l'immeuble [le palais Dobrée] à sa destination nouvelle : transformation difficile qui ne pouvait pas se faire en un jour. La période d'organisation a été forcément très longue, les bâtiments n'étant pas achevés, tout le matériel d'installation, vitrines, etc. restant à créer. Il y

262. Archives du musée Dobrée, Commission administrative 1876-1933, 18 mai 1895.

263. Archives du musée Dobrée, Commission administrative 1876-1933, 19 mars et 8 mai 1896

264. Archives du musée Dobrée, lettre du maire, Alfred Riom, à Eugène Orieux, vice-président de la Commission administrative du musée départemental d'Archéologie, 18 avril 1896.

265. En réalité, il y avait également des collections dans la propriété de Thomas Dobrée au Grand-Blottereau.

266. Lettres de Pitre de Lisle au préfet Georges Prosper Cleiftie, du 12 mars 1896 et du 10 avril 1896, ADLA, 171 T 3, pièces 65 et 162.

267. Hippolyte Durand-Gasselin débloque la somme de $10000 \mathrm{~F}$ pour les travaux qui sont confiés à l'architecte François Le Diberder. 
avait surtout d'immenses difficultés pour transformer en salles de Musée la partie destinée à l'habitation ${ }^{268}$."

Pitre de Lisle agit, là encore, avec méthode et fait procéder "à la fabrication de caisses divisées en compartiments qui réuniront les objets de chaque vitrine de façon à conserver l'ordre méthodique et le classement des collections ". Les vitrines prévues au palais Dobrée ne pouvant accueillir la totalité des objets archéologiques, le conservateur laisse en caisses une partie des collections archéologiques car "les objets sont en assez bon ordre pour pouvoir être consultés au besoin ${ }^{269}$ ".

Le transfert des collections a donc été fait en deux temps, en commençant par les collections archéologiques. Après autorisation de la Ville de Nantes, le transfert de ces collections est réalisé aux frais du Département, en 1896 et jusqu'au printemps 1897. Le conservateur procède ensuite à leur installation, non pas dans le manoir de la Touche, qui n'est pas en état de les recevoir, mais au rez-de-chaussée du palais, l'étage étant réservé aux collections Dobrée, " si remarquables formées par le riche Mécène nantais, et données par lui au Département avec le superbe écrin qui les garde ${ }^{270}$ ". En attendant la rénovation du manoir, les collections lapidaires prennent place dans le jardin :

"En réunissant ainsi dans un même établissement toutes nos antiquités, magnifiquement installées dans les salles et les galeries des Irlandais, avec un vaste jardin pour recevoir les sculptures et les fragments de nos anciens monuments, on créait à Nantes un Musée digne de notre vieille cité bretonne ${ }^{271}$. "

Pour l'archéologie, Pitre de Lisle prend le soin de faire valider par la Commission administrative le plan d'implantation chronologique des collections au rez-de-chaussée du palais :

"Les objets appartenant aux époques les plus récentes, seront placés dans la première salle joignant le grand escalier, les antiquités du Moyen Âge feront suite et les collections des époques primitives se trouveront à l'extrémité du Musée. Il y aurait ainsi une succession chronologique qui aiderait à la clarté du classement ${ }^{272}$."

Pour rendre hommage aux principaux donateurs, il est décidé que les salles où sont placées leurs collections porteront leur nom.

En 1899, le préfet Hélitas et la Ville de Nantes réclament encore que le Département procède, dans les plus brefs délais, à l'enlèvement des encombrantes sculptures de la chapelle renaissance Thomas Le Roy de la Collégiale Notre-Dame, qui décore la chapelle de l'Oratoire et lui sert de narthex. Il est même question de reconstituer cette chapelle dans le jardin. Pesants et encombrants, ces 400 blocs sculptés ne quitteront l'Oratoire qu'en 1990.

268. L'Écho de la Loire, février 1924, nécrologie de Pitre de Lisle du Dreneuc.

269. Archives du musée Dobrée, Commission administrative 1876-1933, 28 avril 1899.

270. Durville, Georges, Catalogue du musée archéologique de Nantes, $2^{\mathrm{e}}$ partie, musée lapidaire (= Cat. 1927), Nantes, imprimerie Dupas, 1927, p. 20.

271. LISLE du DRENEuc, Pitre de, Cat. 1903, p. VIII.

272. Archives du musée Dobrée, Commission administrative 1876-1933, 8 octobre 1895. 
Une fois achevés le transfert des collections de l'Oratoire et l'installation, au rez-de-chaussée du palais, de trois salles d'archéologie, le conservateur entreprend, durant l'été 1897, le transfert des collections Dobrée dans trois salles du $1^{\text {er }}$ étage du palais. Une salle du $2^{\mathrm{e}}$ étage sert de réserve aux objets et documents non exposés.

Le bâtiment étant toujours en travaux, la Commission administrative est obligée de repousser au dimanche 8 janvier $1899^{273}$ la date de l'ouverture au public du rez-de-chaussée du musée proposée par Pitre de Lisle du Dreneuc $^{274}$. Il ne s'agit encore que d'une ouverture provisoire de la partie archéologique, l'inauguration officielle ne devant avoir lieu que lorsque les aménagements intérieurs et extérieurs du palais seront terminés. En fait, on ne conserve aucune trace de l'inauguration officielle des lieux, même pas mentionnée dans le Bulletin de la Société archéologique. On sait seulement que la Société archéologique y tient sa première séance le 10 janvier $1899^{275}$, en attendant l'achèvement des travaux intérieurs du manoir Jean V. Il est vrai que le testament de Thomas Dobrée prohibait toute fête dans le musée qui devait porter son nom.

La publication de Pitre de Lisle du Dreneuc sur cette première présentation n'ayant pas été retrouvée ${ }^{276}$, seuls les articles du Populaire et du Progrès de Nantes datés des 2 et 3 septembre 1898 offrent une description des salles à cette époque. Au rez-de-chaussée, d'ouest en est, les collections de la chapelle de l'Oratoire se déploient sur huit salles : pièces "historiques" et vues du "Vieux Nantes", arts religieux et arts décoratifs, armes, céramiques et briques de différentes époques, collections des "périodes celtique et dolménique " (dont la collection Kerviler), outillage lithique, sculptures en bois ou en pierre du Moyen Âge et de la Renaissance, enfin les collections grecques et étrusques accompagnées des dessins de Gustave Bourgerel, pas encore déposés au musée des Beaux-Arts de Nantes. Si l'on en croit ce descriptif, on est assez loin du classement méthodique et chronologique prôné par le conservateur. Le journaliste précise :

"Tous les objets du musée archéologique n'ont pas encore trouvé place dans les vitrines du palais Dobrée. C'est ainsi que le superbe reliquaire en or de la duchesse Anne, donné au Musée par la Ville, n'est pas exposé actuellement, la vitrine qui doit le contenir n'étant pas encore achevée. "

273. Archives du musée Dobrée, Commission administrative 1876-1933, 13 décembre 1898. 274. Archives du musée Dobrée, Commission administrative 1876-1933, 31 juillet 1897; la Commission parle alors d'une " affaire de deux ou trois mois au plus ". Une "visite inaugurale ", organisée le 15 octobre 1897, est relatée par Marius Vachon, avec photographies (L'Illustration, 2851, 16 octobre 1897), mais il n'en est fait état ni dans le procès-verbal de la réunion de la Commission administrative du musée Dobrée réunie le lendemain, 16 octobre, ni dans celle de la Commission du musée départemental d'Archéologie, réunie le 22 octobre. 275. BSANLI, 40, 1899, p. VIII, 10 janvier 1899. Il ne s'agit que d'une " salle provisoire [...]. On devait nous aménager une salle qui n'est pas encore prête; mais bientôt, nous l'espérons, $M$. [Ludovic] Cormerais [le nouveau président de la Commission administrative du musée Thomas Dobrée] pourra installer la Société d'une façon digne d'elle ".

276. Lisle du Dreneuc, Pitre de, Notice sur le Musée Th. Dobrée, Nantes, Émile Grimaud éd., 40 p., avec illustrations. 
Les antiquités égyptiennes seront, elles-aussi, installées postérieurement $^{277}$.

À l'étage, les collections de Thomas Dobrée se déploient sur cinq salles : d'est en ouest, les onze gravures de Philibert Louis Debucourt et quelques tableaux, dont le paysage de Dupré, les pièces chinoises du Palais d'Été accompagnées d'une crédence italienne, des tableaux flamands et de vitrines d'orfèvrerie, de faïences ou de porcelaines, dans la salle où est enchâssé dans un lambris le portrait posthume du donateur Thomas Dobrée par Paul Émile Chabas, un ensemble de pièces précieuses : les émaux, dont la châsse de saint Calmin, l'aquamanile Le lai d'Aristote, les manuscrits enluminés, des meubles dont la cathèdre et un dressoir aux armes de la reine Anne et des tableaux de peintres primitifs; dans des vitrines murales, fermées par des volets, sont présentées les plus précieuses gravures : Rembrandt, Dürer, Fragonard, Greuze, etc. Resteront à présenter les monnaies et médailles, les autographes et les ouvrages de la bibliothèque. Le bureau du conservateur est situé à cet étage. Rapidement, dès 1901, des estampes et dessins représentant des vues de Nantes et appartenant aux collections de Thomas Dobrée, viennent illustrer la salle du "Vieux Nantes" au rez-de-chaussée du musée archéologique ${ }^{278}$.

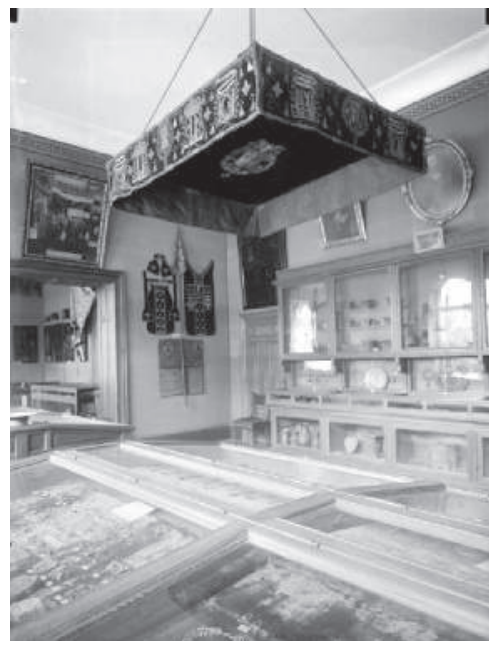

Figure 56 - Musée Dobrée, seconde salle du rez-de-chaussée du palais consacrée aux arts religieux, aux sceaux, etc.

(Fonds de la Société archéologique)

Thomas Dobrée et Pitre de Lisle ont chacun travaillé sur le type de mobilier nécessaire à cette présentation. D'inspiration néogothique ou Renaissance, selon le cas, ce mobilier spécialement conçu : vitrines de

277. Elles ne seront installées qu'en 1909, en même temps que les collections ethnographiques.

278. Archives du musée Dobrée, Commission administrative du musée Thomas Dobrée 1896-1951, 22 août 1901. 
Figure 57 - Le conservateur, Pitre de Lisle du Dreneuc, et son épouse Adrienne dans la bibliothèque du $1^{\text {er }}$ étage du palais Dobrée, devant le portrait posthume de Thomas Dobrée par Paul Chabas, 1898

(fonds de la Société archéologique)

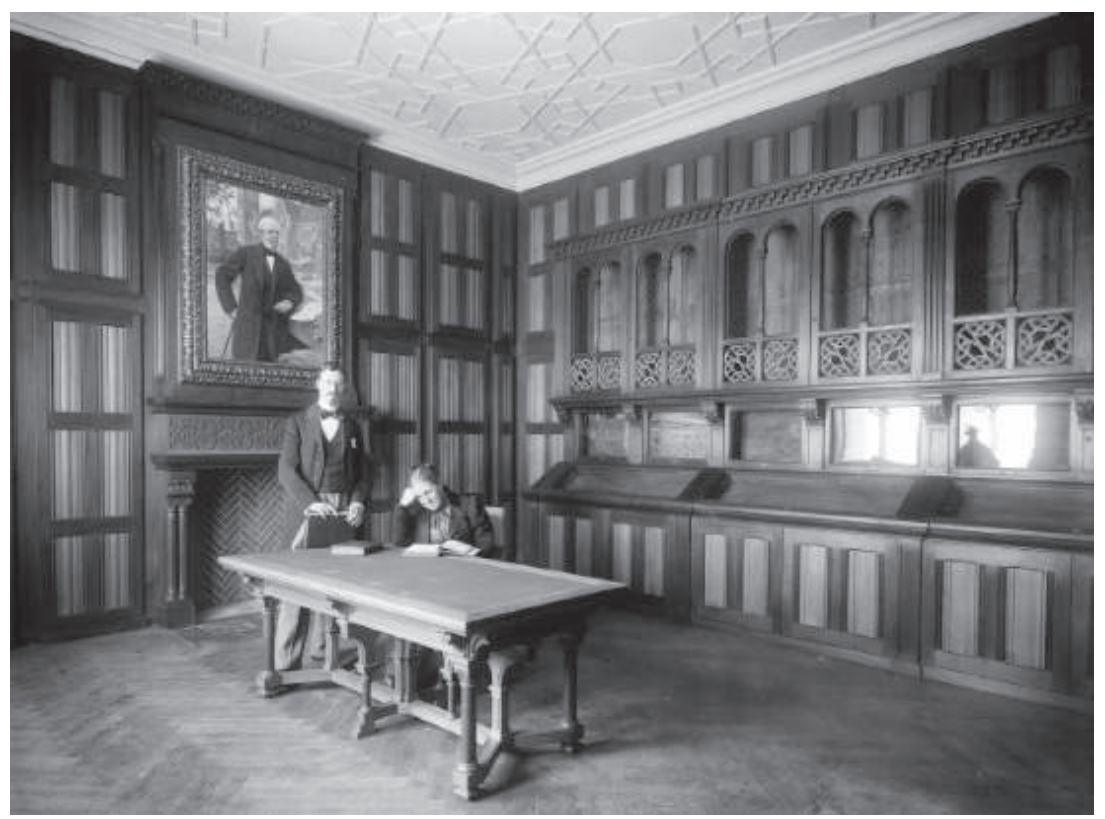

Figure 58 - Entrée du musée départemental d'Archéologie installé au rez-de-chaussée du palais Dobrée, 1906

(fonds photographique du musée Dobrée)

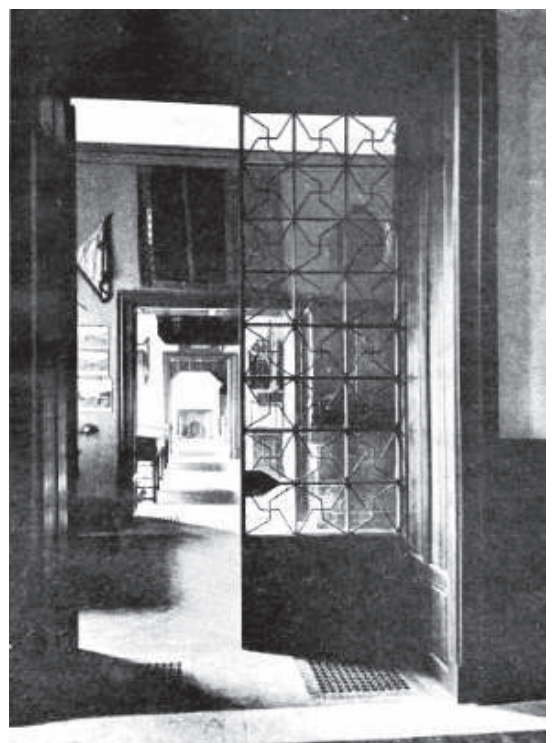


Figure 59. Musée départemental d'Archéologie dans le palais Dobrée, salle I, "Vieux Nantes », 1906

(fonds photographique du musée Dobrée)

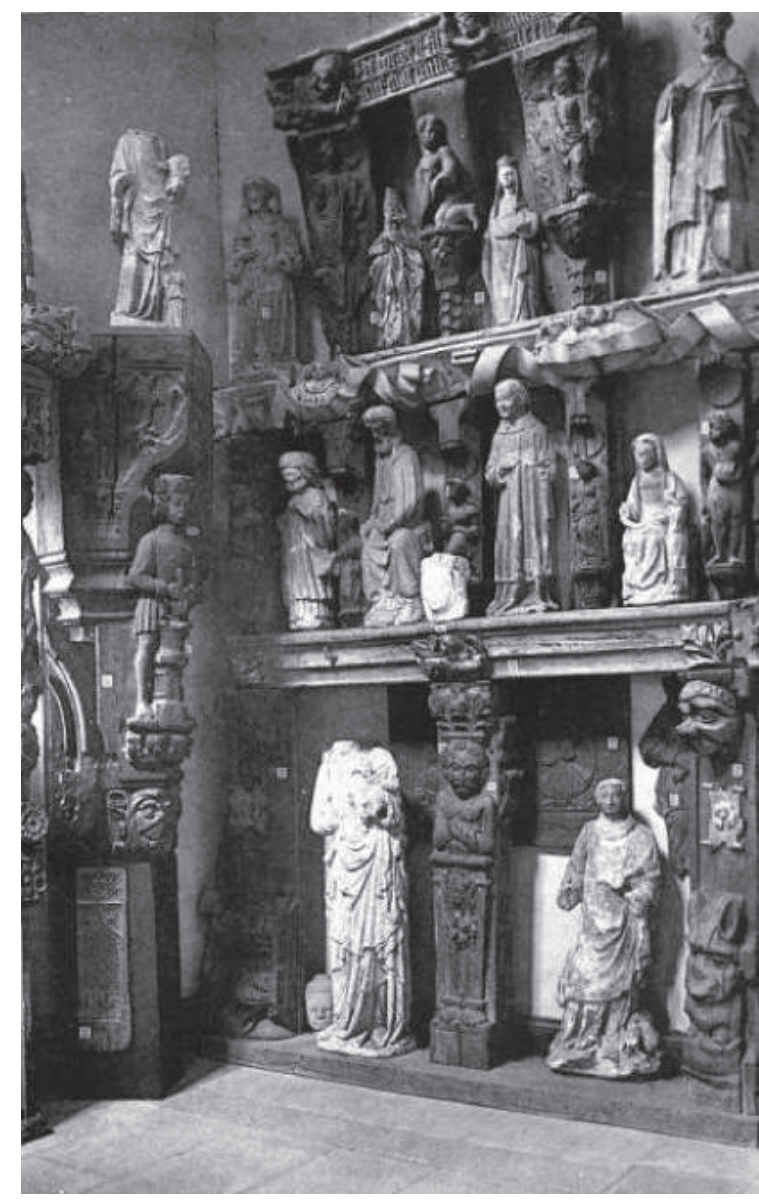

verre et de laiton posées sur des tables de chêne au piétement ouvragé pour les présentations centrales; vitrines unifaces en verre et laiton sur caisson de bois, appliquées contre les murs pour les présentations en périphérie. Des meubles hauts, à claire-voie et grillagés, munis de volets, sont destinés à la présentation des gravures ou des ouvrages. Pour les mobiliers archéologiques placés au rez-de-chaussée, des vitrines-tables, plus simples, au châssis de bois vitré, parfois à double pente, ont été prévues par le conservateur qui avait pu s'inspirer de visites dans plusieurs musées. 
Figure 60. Musée départemental d'Archéologie dans le palais Dobrée, salle II, présentation dense des collections d'armes, 1906 ?

(fonds photographique du musée Dobrée)

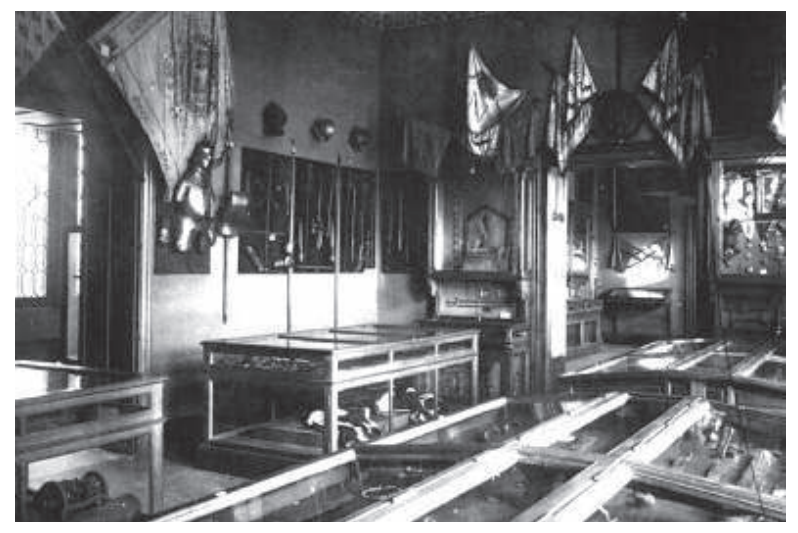

Figure 61. Musée Thomas Dobrée, $1^{\text {er étage du }}$ palais Dobrée, salle II, présentation aérée d'art décoratif francais et chinois, 1906 ?

(fonds photographique du musée Dobrée)

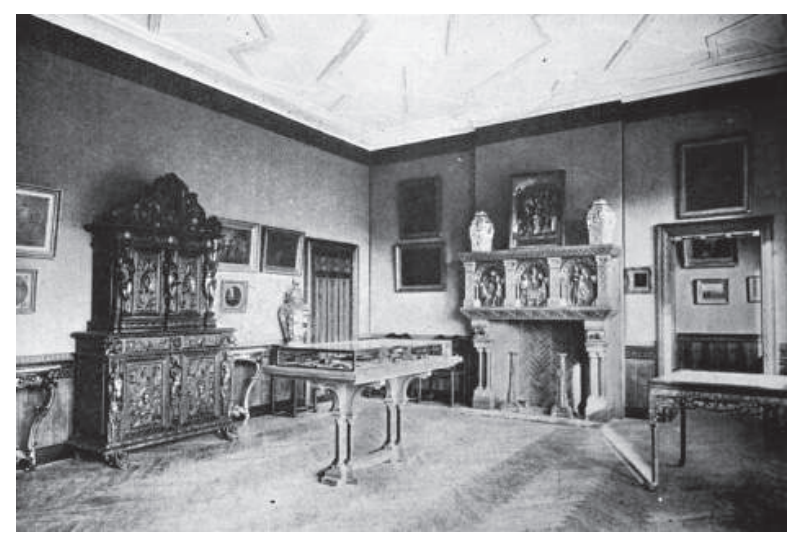

Les catalogues des collections édités entre 1901 et 1906, illustrés de quelques vues de salles, donnent une assez bonne idée du caractère éclectique et foisonnant de cette présentation, très dense pour l'archéologie au rez-de-chaussée, plus aérée à l'étage en raison du type même et du nombre des collections de Thomas Dobrée, beaucoup étant des documents graphiques ou de bibliophilie, donc très fragiles et difficilement présentables.

La Société archéologique ne viendra visiter officiellement ce nouveau musée que le 23 avril 1907, "sous la direction du savant conservateur, M. P. de Lisle ", en présence du président de la Société, le baron Christian de Wismes. Le journal L'Express de l'Ouest s'en fait l'écho ${ }^{279}$.

Sollicité depuis longtemps par la Commission administrative du musée archéologique, Pitre de Lisle travaille à la rédaction de la troisième édition du catalogue des collections archéologiques, qui se sont considérablement enrichies depuis l'édition de 1869 par Fortuné Parenteau, avec,

279. OUVRARD, Léonce, "Visite de la Société au Musée archéologique ", BSANLI, 48, 1907, p. XXVI-XXIX. 
notamment, l'entrée des collections Parenteau, Seidler, Prigent, PerthuisLaurant ou Marionneau. Dès 1895, une commission " auxiliaire " avait d'ailleurs été nommée par la Commission administrative pour la rédaction du nouveau catalogue ${ }^{280}$. Charles Riardant, Xavier Le Lièvre de La Touche et Paul Soullard sont désignés pour aider à l'étude et au classement des monnaies. L'architecte Georges Ferronnière accepte de se charger de la description des sculptures du Moyen Âge et de la Renaissance ${ }^{281}$. En 1901, Georges Durville, le baron Martineau, Paul de Berthou, M. ThibaudNicollière viennent renforcer le comité de rédaction du catalogue. Pour faciliter l'enregistrement des collections et palier les oublis répétés d'enregistrement sur les registres énonciatifs, notamment pour les années 1881 et 1882, en raison du mauvais état de santé de Fortuné Parenteau, Pitre de Lisle propose un enregistrement sur fiches qui simplifiera le classement et facilitera, en outre, la préparation du catalogue imprimé. En 1901, "la première partie du catalogue est prête à donner à l'impression. Malheureusement, les fonds manquent ${ }^{282}$ ". En attendant, comme le conservateur se plaignait de ne pouvoir dresser l'inventaire précis de la collection Parenteau, car " pendant 24 ans, M. Parenteau, conservateur du musée, a récolté simultanément pour le Musée et pour sa collection qui était destinée à en faire partie ", la Commission administrative lut au conservateur un rapport sur la collection Parenteau, " $M$. Parenteau s'étant réservé bon nombre d'objets qu'il n'a pas donnés et dont plusieurs sont figurés et décrits à l'Inventaire archéologique ". Pitre de Lisle propose donc, pour ce catalogue, de suivre la présentation chronothématique des vitrines du rez-de-chaussée du palais Dobrée et de décrire les objets de la collection Parenteau qui y sont présentés dans la période concernée, plutôt que d'en faire un pavé séparé. La publication est décidée en mai $1902^{283}$ et s'achève en $1903^{284}$. Même non illustré, l'apport scientifique de cette troisième édition du catalogue est indéniable et le travail fourni, considérable. Dans son Avis tenant lieu de préambule, Pitre de Lisle explique que «la méthode de classement par séries a le grand avantage de permettre d'inscrire de suite chaque objet qui entre au Musée dans la catégorie à laquelle il appartient; elle est du reste adoptée par nombre de Musées ". Douze séries sont ainsi répertoriées, correspondant aux neuf salles aménagées. Dans chaque série, le classement est chronologique et les vitrines rassemblent soit des ensembles par site, soit des ensembles de collectionneur. Le classement chronologique est plus rigoureux que dans le catalogue

280. Archives du musée Dobrée, Commission administrative 1876-1933, 18 mai 1895. 281. Archives du musée Dobrée, Commission administrative 1876-1933, 4 juillet 1903. 282. Archives du musée Dobrée, Commission administrative 1876-1933, 29 mai 1901. 283. Archives du musée Dobrée, Commission administrative 1876-1933, 5 mai 1902. 284. Archives du musée Dobrée, Commission administrative 1876-1933, 12 mai 1903; deux tirages sont prévus, l'un sans illustrations, à 1000 exemplaires et vendu au prix de 1F10; l'autre, enrichi de 16 planches (8 de l'ancien catalogue Parenteau et 8 photographies), tiré à 500 exemplaires et vendu au prix 1F50. Il ne semble pas que la version illustrée ait été imprimée. 
Parenteau de 1869, même si certains ensembles restent éclatés, comme les armes qui sont classées à l'âge du Bronze, d'une part, et dans une série "armes" de la salle IV, elle-même scindée en trois parties (classement par époque, par catégories et armes défensives) ou encore la série des "bijoux" (salle V), classés chronologiquement, mais auxquels sont associés les émaux, l'orfèvrerie, les objets religieux, les clefs, etc. La nécessité de conserver des vitrines de collectionneurs (Parenteau, Seidler, Kerviler, par ex.), nécessairement diachroniques, a grandement limité la rigueur du classement chronologique. Si le catalogue de 1869 présentait 2250 objets ou ensembles, les dépôts monétaires ou d'argenterie ne comptant, par exemple, que pour un seul numéro d'inventaire, le catalogue de 1903 en dénombre 10833. Les enrichissements considérables effectués entre 1882 et 1903 ne justifient pas, à eux seuls, cet accroissement numérique : le conservateur a dû faire un important travail d'inventaire rétrospectif ${ }^{285}$. Grâce, sans doute, au système de fiches qu'il a lui-même mis en place dès 1892, il peut désormais individualiser bon nombre d'objets. Ce catalogue reste encore aujourd'hui le document le plus important concernant les collections entrées au XIX ${ }^{\mathrm{e}}$ siècle. À partir de 1903, le conservateur, avec l'accord de la Commission administrative, n'inscrit plus ses acquisitions dans le Registre énonciatif du musée, mais sur les fiches manuscrites et sur un exemplaire spécial de son catalogue qu'il complète des nouveaux numéros d'entrée ${ }^{286}$, par catégories "à la suite du catalogue imprimé, et non sur le registre d'entrées où les pièces les plus diverses se trouveraient forcément inscrites sans ordre. Le futur catalogue sera ainsi tout préparé" . Malheureusement le musée n'a conservé ni les fiches de Pitre de Lisle du Dreneuc, ni son catalogue enrichi, archives fondamentales établissant l'origine, la nature et le numéro d'inventaire des collections acquises à partir de 1903, qui, pour ce qui concerne l'archéologie, ne peuvent être connues que par les procès-verbaux des réunions de la Commission administrative et des séances de la Société archéologique, pour les seuls objets dont l'acquisition a été soumis à l'avis de la Commission. Seules les collections lapidaires feront encore l'objet d'un catalogue, en $1927^{287}$.

Sans doute trop occupé par la charge des deux musées, le conservateur ne participe plus, depuis 1895, aux recherches archéologiques à Nantes et dans le département, et n'assiste que très rarement aux séances de la Société archéologique. L'abbé, puis chanoine Georges Durville a pris le relai et suit les travaux archéologiques. Il travaille avec Pitre de Lisle du Dreneuc au moins depuis 1901, quand il est chargé par la Commission administrative d'étudier l'installation des collections lapidaires dans les trois salles du rez-

285. Pour rappel, le nombre d'objets ou d'ensembles enregistrés à la mort de Fortuné Parenteau est de 1323; il passe à 6656 à la fin de 1902, date à laquelle Pitre de Lisle ne tient plus le Registre énonciatif, alors que le catalogue de 1903 présente 10833 objets ou ensembles!

286. Archives du musée Dobrée, Commission administrative 1876-1933, 29 mai 1901 et 9 mai 1906.

287. DuRVILLE, Georges, Cat. 1927. 
de-chaussée du manoir Jean $\mathrm{V}^{288}$, alors en travaux. Il contribue également à la rédaction de plusieurs catalogues du musée Thomas Dobrée. En effet, entre 1901 et 1906, Pitre de Lisle, aidé par de nombreux collaborateurs dont Georges Durville, assure ou coordonne l'énorme travail de publication des collections du musée Thomas Dobrée ${ }^{289}$.

En 1903, Pitre de Lisle publie le catalogue de vente des collections archéologiques qu'il a constituées avec son frère Georges ${ }^{290}$, divisant ainsi de manière regrettable des ensembles archéologiques déjà donnés au musée par son conservateur en 1887. Sans doute motivée par des difficultés financières, cette vente, dont le caractère discutable n'a pas dû échapper aux contemporains, n'est pas même évoquée dans les procès-verbaux des réunions de la Société archéologique et des Commissions administratives des deux musées. La Commission administrative espérait peut-être un don ou les moyens alloués ne permettaient sans doute pas cet achat : quoique cette collection soit essentielle pour le musée, il ne se porte pas acquéreur. Après des pourparlers avec des universités américaines, et sur l'intervention de Gustave Paille, Pitre de Lisle vend le 28 octobre 1903 l'intégralité de sa collection, au prix de 5000 F, au marquis Pierre Auguste de Montaigu, membre correspondant de la Société archéologique, vice pré-

288. Archives du musée Dobrée, Commission administrative 1876-1933, 21 juin 1901.

289. Dans l'introduction du Catalogue général des collections du Musée Th. Dobrée, Pitre de Lisle précise en 1901 que "la description des dix mille objets de ces différentes séries exigeait un travail infini, très long, surtout très minutieux à cause du caractère exceptionnel de beaucoup de pièces inédites ou peu connues [...]. Et, cependant, le temps pressait, car il était indispensable de dresser, à l'aide d'un catalogue détaillé et complet, l'inventaire de ce qui devait constituer le Musée Dobrée. De 1901 à 1906, en moins de cinq années, sept volumes de catalogue ont été publiés [...]. Je ne crois pas qu'il y ait de Musée où le travail d'inventaire ait été poussé aussi activement, et cette opinion nous est confirmée par l'Annuaire publié par les soins du Ministère. Nous y voyons que, parmi les Musées de France, il y en a encore 246 qui n'ont pas de catalogue " : LISLE DU DRENEUC, Pitre de, avec le concours de Polain, Louis, Catalogue des Autographes du Musée Th. Dobrée, Nantes, Émile Grimaud (éd.), 1901; Polain, Louis, Catalogue de la Bibliothèque du Musée Th. Dobrée, Imprimés, II, Paris, imp. J. Dumoulin, 1903; Durville, Georges, Catalogue de la Bibliothèque du Musée Th. Dobrée, Manuscrits, I, Nantes, imp. Moderne, Joubin et Beuchet frères, 1904; LISLE DU Dreneuc, Pitre de, Catalogue sommaire de la Bibliothèque du Musée Th. Dobrée, Nantes, imp. Moderne, Joubin et Beuchet frères, 1905; LISLE Du Dreneuc, Pitre de, Catalogue général des collections du Musée Th. Dobrée, Nantes, imp. Moderne, Joubin et Beuchet frères, 1906 (= Cat. 1906).

290. Bretagne. Catalogue de la collection archéologique de M. Georges de Lisle du Dreneuc et M. P. de Lisle du Dreneuc, Nantes, imp. Moderne, Joubin et Beuchet frères, 1903. Dans une brève introduction, les deux frères expliquent : «Cette collection comprend les objets recueillis dans nos fouilles et nos explorations en Bretagne, principalement en LoireInférieure. Nous avons tenu à décrire et à noter ces pièces de façon à ce qu'elles indiquent avec précision le lieu de la trouvaille, pour servir à l'étude archéologique de la région. Nos fouilles ont été décrites dans des Notices qui forment le complément de ce catalogue. " Dans sa lettre du 26 août 1903 à Gustave Paille, l'archéologue du marquis de Montaigu, Pitre de Lisle précise : "Tous [les objets] sont de provenance authentique et certaine puisque nous les avons recueillis sur place ", musée Dobrée, "papiers Paille", archives de la collection Dommée, musée Dobrée. 
sident du Conseil général et député de la Loire-Inférieure ${ }^{291}$. Elle venait compléter les collections, déjà fort riches, du marquis de Montaigu. Le musée privé du château de La Bretesche, en Missillac, reste ouvert jusqu'en 1959. Un malaise demeure sans doute car, dans son compte-rendu de la visite du musée de la Bretesche par la Société archéologique, en 1908, le président Alcide Dortel décrit les vitrines et leur contenu mais ne cite pas une seule fois les collections vendues par Pitre de Lisle alors qu'il s'attarde sur les découvertes de Léon Maître et du commandant Martin à Clis (près de Guérande, Loire-Atlantique) ${ }^{292}$. Pourtant cette collection ne sombre pas dans l'oubli et reste dans la mémoire des archéologues régionaux puisqu'en 1965, le préhistorien Pierre Roland Giot évoque encore "l'importante collection préhistorique et archéologique de Lisle du Dreneuc ${ }^{293}$ ". Dans les années soixante, la collection est dispersée par le marquis Philippe de Montaigu et ce qu'il en reste est acheté par l'architecte nazairien Claude Dommée, amateur d'archéologie. 90 ans après la vente de Pitre et Georges de Lisle du Dreneuc, ce sont 833 pièces ou ensembles (sur 701 numéros inscrits au catalogue de la vente) qui sont généreusement données en 1993 au musée Dobrée par Paulette Dommée, l'épouse du dernier propriétaire, permettant ainsi de rassembler de nouveau des séries dispersées ${ }^{294}$. L'examen

291. SANTRot, Jacques, avec le concours de Blain, Hugues-François, " Musée Dobrée, acquisitions 1993 ", BSANLI, 129, 1993, p. 269-278. SANTROT, Jacques, " Musée Dobrée, acquisitions 1994 ", BSANLI, 130, 1994-1995, p. 249-262. Comme il l'écrit à Gustave Paille, le conservateur est satisfait que sa collection soit achetée par un collectionneur local, membre de la Société archéologique comme lui, "mais l'espoir de voir ma collection faire retour ici me séduit beaucoup ", lettre du 26 octobre 1903, musée Dobrée, "papiers Paille", archives de la collection Dommée, musée Dobrée.

292. DoRTEL, Alcide, « Excursion du lundi 18 mai 1908 », BSANLI, 49, 1908, p. XXVIII-XXXI.

293. Lettre de Pierre Roland Giot, directeur de la Circonscription préhistorique de Bretagne et des Pays de La Loire, adressée le 3 avril 1965 à l'Administrateur civil chargé du Bureau des Fouilles et Antiquités, à propos de la vente du château de La Bretesche et de ses collections, en 1964.

294. BLaIN, Hugues-François, La collection Dommée, étude de la formation d'une collection privée et catalogue des objets qui se rapportent à l'Antiquité, mémoire de maîtrise d'histoire ancienne de l'Université de Nantes, 1994-1995 : huit cent trente-trois pièces ou ensembles proviennent de la collection personnelle de Pitre de Lisle, dont $91 \%$ appartiennent à la Préhistoire, $2 \%$ à la Protohistoire, $5 \%$ à l'Antiquité et $2 \%$ sont des pièces ethnographiques. La collection donnée au musée en 1993 par Paulette Dommée provient pour une grande partie de la Collection archéologique de M. Georges de Lisle du Dreneuc et M. P. de Lisle du Dreneuc dont le catalogue a été publié par l'Imprimerie Moderne, à Nantes en 1903, 19 pages, 701 numéros. Cette collection est presque intégralement conservée puisque seulement une vingtaine de numéros manquent aujourd'hui. Elle fut complétée ultérieurement par ses propriétaires successifs, par l'adjonction de séries de provenance diverse, non cataloguées, dont certaines furent ultérieurement dispersées. Vendue en 1903 au marquis Pierre-Auguste de Montaigu, cette collection fut augmentée des acquisitions faites par lui avec la collaboration rémunérée de l'archéologue Gustave Paille, chargé de prospecter et de fouiller en Loire-Atlantique et en Morbihan, entre 1897 et 1906 probablement. Propriété des Montaigu, cet ensemble fut longtemps conservé au château de la Bretesche, en Missillac (Loire-Atlantique) où il constituait un musée privé. Il fut acquis à Philippe de Montaigu vers 1962 par Claude Dommée, architecte. Divers objets, qui lui ont depuis été associés, proviennent des ramassages, des fouilles ou des 
minutieux de cette collection montre que, Pitre de Lisle, à la fois collectionneur passionné et conservateur de musées, n'a pu résister à attribuer à sa collection personnelle, de "belles" pièces distraites des séries acquises par le musée. Cette collection personnelle des de Lisle montre une double origine : les objets qui la composent proviennent, pour une part, des fouilles des deux frères, pour une autre, de "prélèvements à la source" dans les collections acquises pour le musée départemental d'Archéologie. Résultat naturel de leurs activités professionnelles, les objets fouillés dans les sites préhistoriques ont été scindés par les deux inventeurs lors du don d'une partie de leur collection au musée archéologique en 1887 : ils ont conservé les plus belles pièces. On retrouve ainsi dans la collection Dommée, le complément du produit de fouilles des sites paléolithiques de Loire-Inférieure de Montbert (L'Ouchette et le Pas-Chalène), Saint-Géréon (L'Étranglard), La Haie-Fouassière (Bégrol et Le Breil), d'Ille-et-Vilaine (Le Mont-Dol), des grottes de Dordogne (La Madeleine à Tursac, et Cro-Magnon aux Eyzies-de-Tayac), les objets néolithiques trouvés dans les mégalithes de La Roche à Donges, du Grand Carreau Vert à Saint-Michel-Chef-Chef, de la Couronne Blanche au Petit-Auverné, ou encore les objets gallo-romains issus des fouilles de Pitre aux Cléons, pratiquées juste avant les importantes fouilles de la villa par Félix Chaillou entre 1882 et 1894, par exemple. Plus grave : des pièces ont été "prélevées" au moment de leur entrée, dans les collections mêmes du musée : quelques objets du bassin de Penhoët, à Saint-Nazaire, données au musée par Kerviler, l'une des neuf haches polies appartenant à un dépôt rare découvert à La Chapelle-Basse-Mer ${ }^{295}$, enfin, la plus belle de "la cinquantaine " des exceptionnelles pointes de flèche ogivales en silex (dont 34 aujourd'hui conservées) du tumulus du Bronze ancien de Tossen-Kergourognon en Prat (Côtes-d'Armor), dont, en 1892, il a négocié l'achat par le musée à l'abbé Prigent leur inventeur ${ }^{296}$.

Depuis 1905, la sécurité du musée et la protection des collections exposées agitent la communauté scientifique locale. Des bruits circulent sur un projet de vol dans le musée; tous les objets en or sont sortis des vitrines et remplacés par des moulages et l'on ajourne même la nouvelle présentation

achats de Claude Dommée, qui consacrait des loisirs à l'archéologie avec ses amis, avec l'horloger-bijoutier de Nivillac, Jean Guillotin, et avec le Dr Jean Méloche, de Saint-Nazaire, en particulier. La collection Dommée fut progressivement recueillie par Jacques Santrot entre août 1993 et mars 1995. Sur la constitution de la collection Dommée et sur la composition de la collection donnée au Musée Dobrée, voir SANTROT, Jacques, « Musée Dobrée, acquisitions 1993 ", BSANLA, 1993 (1994), 129, p. 267-277, avec répertoire alphabétique des origines des objets pour les 481 premiers numéros, et SANTROT, Jacques, "Musée Dobrée, acquisitions 1994 ", BSANLA, 130, 1994 (1995), avec répertoire alphabétique de l'ensemble des origines connues.

295. BSANLI, 4, 1864, p. 11, 15 mars 1864. BAUdOUIN, Marcel, " Les cachettes ou dépôts rituels de haches polies en Loire-Atlantique ", BSANLI, 62, 1922, p. 83-100. Marcel Baudouin émet un doute sur le nombre de haches découvertes en 1864 : neuf selon l'agriculteur inventeur de la découverte, huit selon Pitre de Lisle...

296. Il a prélevé la plus belle pièce d'un ensemble d'une cinquantaine de pointes de flèche découvert en 1880 . 
du "reliquaire" en or émaillé du cœur d'Anne, reine de France et duchesse de Bretagne, derrière une grille en fer dans la tourelle sud donnant sur la bibliothèque, au premier étage du palais ${ }^{297}$. Dès juillet 1906, 17 monnaies sont volées au musée, dont 5 monnaies gauloises en or ${ }^{298}$. Une seconde et dramatique effraction est mise en échec en 1907 par le gardien de nuit mais il décède un an plus tard des suites de ses blessures ${ }^{299}$. Les objets précieux sont réinstallés en 1909 dans des vitrines plus sûres.

L'autre chantier important du conservateur est l'installation des collections lapidaires, égyptiennes et ethnographiques dans le manoir de la Touche. En 1911, les collections égyptiennes sont partiellement installées au rez-de-chaussée du palais mais dans une salle humide, inadaptée et les vitrines pour installer les momies ne sont toujours pas réalisées ${ }^{300}$. En 1903, l'architecte Georges Ferronnière est chargé, par la Commission administrative du musée archéologique et avec l'accord du conservateur, de l'installation des collections lapidaires dans les salles du rez-de-chaussée du manoir ${ }^{301}$. L'année suivante Georges Ferronnière donne un aperçu des travaux nécessaires pour achever d'organiser les salles du manoir Jean $\mathrm{V}$ tandis que Pitre de Lisle fait réaliser des moulages des inscriptions galloromaines encastrées dans la galerie de l'hôtel de ville pour pouvoir les exposer dans le manoir ${ }^{302}$. En 1910, le conservateur établit un rapport sur l'installation des collections lapidaires pour justifier sa demande de subvention au Conseil général. Il explique que

"Jusqu'ici les sculptures, les inscriptions, les édicules réunis depuis un demi siècle n'avaient jamais pu être exposés complètement faute d'un local suffisant pour les recevoir, et cependant se sont les pièces les plus intéressantes de nos collection, les archives lapidaires de la vieille cité nantaise ${ }^{303}$."

297. Archives du musée Dobrée, Commission administrative du musée archéologique 1876-1933, 28 mars 1904, 21 juin 1905, 27 mars 1907, 20 février 1908, et Commission administrative du musée Thomas Dobrée, 1896-1951, 17 mars 1902.

298. BSANLI, 47, 1906, $3^{\text {e }}$ et $4^{\mathrm{e}}$ trimestre, p. XLI, 16 octobre 1906.

299. Archives du musée Dobrée, Commission administrative du musée Thomas Dobrée 1896-1951, 19 mars 1907 : "Lors de la tentative de cambriolage [...] le $S^{r}$ Guillaume, beaupère du gardien Gibouin, s'est très bien conduit : ayant entendu du bruit lorsqu'un des voleurs eut pénétré dans le monument par l'imposte vitrée de la porte près de la chambre, il sortit et aussitôt fut frappé à la tête par le malfaiteur d'un coup de barre de fer qui l'abattit sans connaissance. Craignant d'être pris les voleurs se sauvèrent. "Âgé de 67 ou 68 ans, le gardien de nuit Guillaume meurt un an plus tard, "victime de son zèle à défendre le Musée ": archives du musée Dobrée, Commission administrative du musée Thomas Dobrée 18961951, 31 décembre 1907 et 20 février 1908. Le poste de gardien de nuit du musée Thomas Dobrée est alors supprimé.

300. Archives du musée Dobrée, Commission administrative du musée Thomas Dobrée, 16 mars 1911.

301. Archives du musée Dobrée, Commission administrative du musée archéologique 1876-1933, 4 juillet 1903.

302. Archives du musée Dobrée, Relations musée-mairie de Nantes 18, lettre du maire Paul Émile Sarradin au conservateur, 9 mai 1904.

303. Archives du musée Dobrée, rapport de Pitre de Lisle du 30 juin 1910. 
Les sculptures sont transportées dans le manoir mais les scellements ne sont pas faits; la restauration et l'installation de la cheminée monumentale de l'Évêché cédée par la Ville ${ }^{304}$ ne peuvent être réalisées sans un budget spécifique :

"Cette mesure [le crédit de $1200 \mathrm{~F}$ demandé] est d'autant plus urgente que le manoir, non utilisé jusqu'ici, est exposé à de fréquentes dégradations, comme en témoignent les nombreux vitraux brisés à coups de pierre par les gamins du voisinage. L'ouverture du musée lapidaire fera cesser cet état d'abandon très préjudiciable au manoir de Jean V [appellation alors usuelle du manoir de la Touche]. "

La rédaction du catalogue est, semble-t-il, presque terminée :

"Il guidera les visiteurs parmi ces antiquités remontant jusqu'aux origines de notre cité et se succédant jusqu'au XviII siècle. Pour donner un aperçu de nos richesses, citons, ça et là, parmi les antiquités romaines de Nantes, le monument triomphal de l'empereur Aurélien avec un superbe bas-relief représentant le combat d'Achille et de Penthésilée, découvert au Bouffay, des statues de sphinx, les divinités romaines, les stèles, les inscriptions de l'ancien Portus Namnetensis. Parmi les antiquités chrétiennes, les beaux marbres de la cathédrale de saint Félix, dont les chapiteaux rappellent le style archaïque de la basilique de sainte Sophie, la grande tombe en marbre rose des Pyrénées de saint Donatien et saint Rogatien [cuve de sarcophage fouillée en 1873, rentrée dans les collections du musée en 1891, puis déposée en 1943 dans la basilique qui leur est consacrée], le tombeau du duc Jean III avec ses fines arcatures gothiques, le monument de Gilles de Retz, l'écusson de pierre sculpté de la duchesse Françoise d'Amboise, les fines sculptures de la Renaissance de la Collégiale, etc., etc. "

Cette même année, Georges Ferronnière expose à la Société archéologique sa proposition de classement et de présentation des vestiges lapidaires dans le manoir ${ }^{305}$. En 1911, Georges Ferronnière souhaite qu'on intègre dans la seconde salle du rez-de-chaussée du manoir le moulage du baptistère de l'Évêché ${ }^{306}$. Il n'a malheureusement pas été conservé.

En 1913, les sols du rez-de-chaussée du manoir doivent encore être réparés pour permettre l'ouverture du "Musée lapidaire" et une subvention est demandée pour l'impression de son catalogue ${ }^{307}$. Sans doute ces salles ont-elles été ouvertes au public avant l'installation réalisée par

304. Archives du musée Dobrée, Relations musée-mairie de Nantes, lettre du maire Paul Bellamy au conservateur, 28 avril 1910. Trop importante pour être présentée au rez-dechaussée du manoir, cette cheminée sera finalement restituée à la Ville pour être installée au château : archives du musée Dobrée, Commission administrative du musée Thomas Dobrée, séance 31 août 1923.

305. BSANLI, 51, 1910, p. XXXVIII, 5 juillet 1910.

306. Archives du musée Dobrée, Commission administrative du musée archéologique, 16 mars 1911.

307. Archives du musée Dobrée, Commission administrative du musée archéologique 1876-1933, 8 mars 1913. Il n'y aura plus de compte-rendu de séance de la Commission administrative du musée archéologique jusqu'à la mort du conservateur Pitre de Lisle du Dreneuc (1924) ; elle s'est cependant réunie une autre fois en 1920. 
Georges Durville en 1927, consacrée par l'édition de son catalogue du Musée lapidaire ${ }^{308}$ dans lequel le nouveau conservateur décrit le travail de l'architecte Ferronnière : "Chargé tout particulièrement, par M. P. de Lisle, de l'aménagement du musée lapidaire, cet éminent professeur [il est professeur de l'université catholique d'Angers] a déployé tout à la fois dans cet aménagement, et ses qualités d'architecte et ses connaissances d'archéologue. " Il a consacré la salle I, grande salle ouest du rez-de-chaussée du manoir, à la "reconstitution" du bas-relief du Bouffay, la salle II présente une portion de la cathédrale de saint-Félix et, enfin, la salle III une fenêtre de la cathédrale du XII ${ }^{\mathrm{e}}$ siècle, les clefs de voûte (au plafond), les sablières (sur le pourtour de la salle). Il considère que "si l'examen détaillé des pièces est difficile [en raison de la hauteur de leur accrochage], en revanche l'effet général de décoration, compense, en partie, cet inconvénient ". Parallèlement à cette longue installation des collections dans le manoir, Georges Ferronnière travaille à la rédaction du catalogue : "Le travail, commencé avant 1911, fut fait en partie, et eut même un commencement d'impression. Mais, vint la guerre; survint la mort prématurée de M. Ferronnière, et tout resta en chantier. "Un an avant sa mort, Pitre de Lisle a demandé à Georges Durville de reprendre le travail sur le catalogue, mais vingt ans plus tard, il était entièrement à recomposer. En outre, le manuscrit du catalogue fut réclamé par l'épouse de Georges Ferronnière et Georges Durville dut "songer à un travail nouveau ". Il optera pour une présentation chronologique : une salle consacrée aux antiquités galloromaines (114 pièces), une autre aux antiquités mérovingiennes et carolingiennes (100 pièces) et une salle du XI ${ }^{\mathrm{e}}$ au XIX ${ }^{\mathrm{e}}$ siècle ( 479 pièces) ${ }^{309}$.

Pendant l'installation du musée lapidaire, Pitre de Lisle ne reste pas inactif : il reprend l'installation des salles du rez-de-chaussée et de l'étage du palais, pour le musée archéologique comme pour le musée Thomas Dobrée. Il rédige en 1910 le règlement du musée Thomas Dobrée ${ }^{310}$ et travaille à la rédaction d'un nouveau Guide du musée archéologique et du musée Thomas Dobrée dont il faut sans doute dater l'édition de $1922{ }^{311}$. Il y fait une description très précise des salles, dont il a renouvelé la présentation, et des objets remarquables : le Vieux Nantes et la salle Petit (dessins), la salle des sceaux, des jetons et des souvenirs révolutionnaires, la salle F. Parenteau avec les collections des guerres de Vendée, les émaux, arts religieux, collections de clefs, mortiers de bronze, bijoux, orfèvrerie, la salle des armes, pour laquelle Pitre de Lisle annonce que : "Cette salle doit prendre le nom de Salle de Rochebrune, la collection du célèbre amateur

308. DuRVILLE, Georges, Cat. 1927, p. 21-22.

309. Ibidem, p. 26.

310. Lisle Du Dreneuc, Pitre de, Règlement du Musée T. Dobrée, Nantes, imp. Dupas, 1910.

311. LisLe Du Dreneuc, Pitre de, Guide du musée archéologique et du musée Thomas Dobrée, s.d., 36 p., non illustré. Archives du musée Dobrée, Commission administrative du musée Thomas Dobrée, 13 juin 1922 : "Un guide du musée va être imprimé, le catalogue actuel étant épuisé. La dépense s'élève à $950 \mathrm{~F}$." 
y sera placée et la disposition actuelle sera alors modifiée 312 ": depuis une dizaine d'années, il est en négociation, et parfois en concurrence, avec le comte Raoul de Rochebrune ${ }^{313}$. Après la salle d'armes, la salle gallo-romaine a une partie consacrée au site de Rezé; elle expose également les décors d'églises mérovingiennes et la tombe gauloise de Châtillon-sur-Indre; la salle du Bronze, outre les armes de l'âge du Bronze draguées en Loire ${ }^{314}$, possède des vitrines pour les dépôts métalliques, et notamment, celui de la Prairie de Mauves; ensuite la salle des collections Kerviler et Gustave Bord, avec le "chronomètre préhistorique" de Penhoët; une salle qui lui est chère : celle des armes de pierre dans laquelle il expose, dans un but pédagogique, à la fois les armes ethnographiques océaniennes, les outils lithiques danois de la collection de Charles Seidler et les outils préhistoriques, locaux ou non. Dans la galerie nord du rez-de-chaussée du palais, dans des vitrines murales, il présente les collections grecques, étrusques et "phéniciennes", ainsi que les céramiques et verreries jusqu'au XIX ${ }^{\mathrm{e}}$ siècle, puis la collection égyptienne de Frédéric Cailliaud et enfin la salle de la collection d'ethnographie éthiopienne de Georges Porquier. Le parcours

312. Ibidem, p. 11. Le projet du don de la collection du comte Raoul de Rochebrune au musée d'archéologie est évoqué depuis le 19 mars 1896. En fait, Raoul de Rochebrune fera un legs, échu en 1926, à la mort de sa femme, mais délivré en 1930.

313. Depuis 1896, Raoul de Rochebrune, qui deviendra membre de la Commission administrative du musée départemental d'Archéologie le 25 mai 1909, est en négociation avec ce musée au sujet, notamment, des objets de la soi-disant " tombe d'un légionnaire romain " trouvée à Jard-sur-Mer (Vendée) par le collectionneur vendéen. En échange du don de ces objets au musée, il demande une très belle épée de l'âge du Bronze et un rare étrier viking trouvé en Loire. Après accord du préfet de Loire-Inférieure, Georges Cleiftie, la Commission administrative du musée accepte l'échange, qui n'a sans doute, heureusement, pas eu lieu : l'invention de la tombe du légionnaire de Jard-sur-Mer est une "forgerie" : archives du musée Dobrée, Commission administrative du musée dépaartemental d'Archéologie 1876-1933, 19 mars 1896 : SANTROT, Jacques, "La "sépulture" d'un légionnaire romain : pièces d'armement antique à Jard-sur-Mer (Vendée) ", dans 150 années de découvertes archéologiques en Vendée, La Mort et le Sacré, VITAL, Christophe (dir.), cat. d'expo., galerie du Puy-du-Fou, Les Épesses (Vendée), Thonon-les-Bains, Conservation départementale des musées de la Vendée, 1990, p. 188-191. Autre exemple des méthodes pratiquées par Raoul de Rochebrune : Pitre de Lisle et Raoul de Rochebrune ont un "rabatteur" commun qui arpente les quais de la Loire pour acheter les objets dragués dans le fleuve : le gardien chef du musée, Rémy Gibouin, qui propose ses services au plus offrant. Le 21 novembre 1902 par exemple, Gibouin écrit à Raoul de Rochebrune : " Je viens de trouver une superbe épée de bronze [...] L'ouvrier qui l'a trouvé ne veut pas me la confier; je suis allé chez lui la photographier. Cette épée a été trouvée dans la Loire, en face $S^{\text {te }}$ Anne, presque dans le port de Nantes [...]. Sur la nombreuse collection qui existe au Musée, il n'y en a pas de semblable. Personne ne la connaît, que moi; je vous en donne la préférence sur le Musée Dobrée. Quant au prix, on en veut $200 \mathrm{~F}$ plus 10 \% que je demande à Monsieur le comte pour moi. Je vous demande la discrétion la plus absolue ", archives du musée Dobrée, fonds Rochebrune, boîte $1, \mathrm{n}^{\circ} 10$, lettres 1-5.

314. Pitre de Lisle, dans son Guide du musée archéologique, p. 15, rappelle à ce sujet que « lorsque je fus nommé Conservateur du Musée, je m'appliquai à suivre les travaux exécutés en Loire, et aidé du gardien-chef Gibouin, très actif et très habile chercheur, nous sommes arrivés aujourd'hui au chiffre de trente-cinq épées de bronze et je crois qu'aucun Musée de France n'en possède autant ni de si variées". 
Fortuné Parenteau (1814-1882) et Pitre de Lisle de Dreneuc (1846-1924)...

Figure 62 - Manoir de la Touche (ou Jean V), façades nord et ouest vers 1930

(fonds Société archéologique)

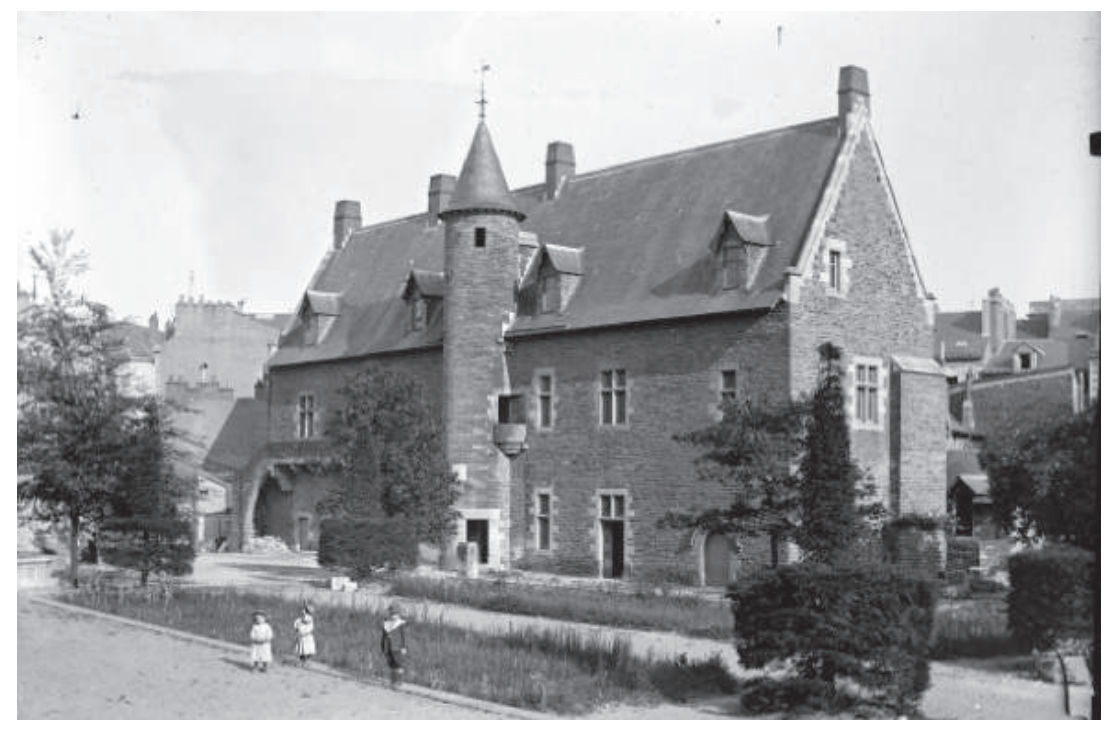

Figure 63 - Musée lapidaire au rez-de-chaussée ouest du manoir de la Touche, salle I, entre 1924 et 1927

(fonds Société archéologique)

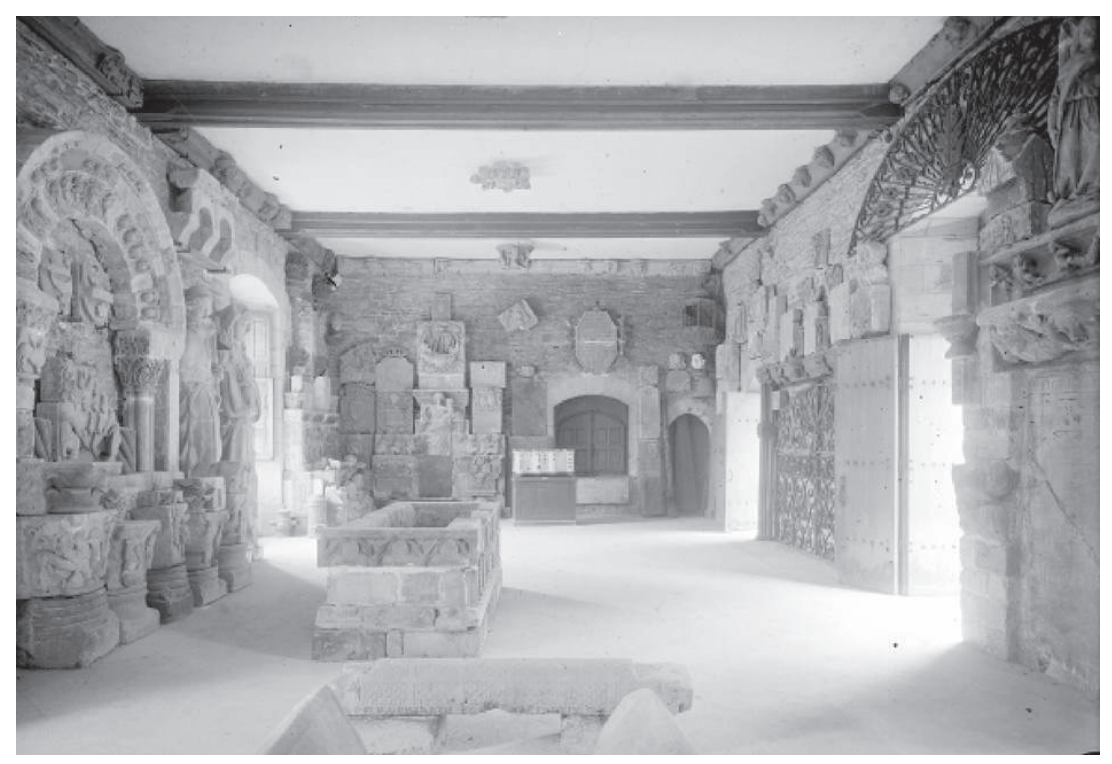


Figure 64 - Musée départemental d'Archéologie, galerie des collections grecques et étrusques, rez-de-chaussée du palais Dobrée, vers 1922

(coll. Louis Bouquet)

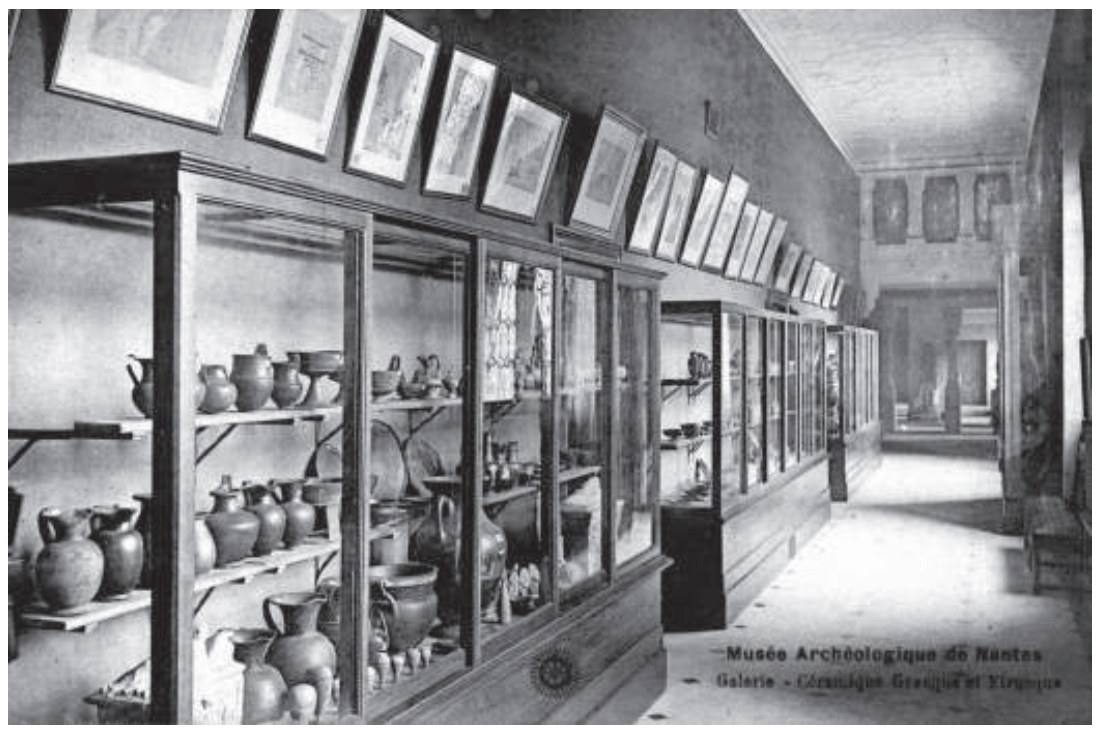

se termine par la salle des bois sculptés provenant des édifices religieux et des maisons de Nantes à pans de bois.

Ce guide sera la dernière publication de Pitre de Lisle du Dreneuc. Sa santé se dégrade depuis plusieurs années ${ }^{315}$. En 1910, il a dû se séparer de sa propriété du Fief, à Sautron, et il a perdu un fils à la guerre de 1914-1918 ${ }^{316}$. S'il ne prend plus guère part aux séances de la Société $\operatorname{archéologique~}^{317}$, Pitre de Lisle assiste, néanmoins, aux séances de la Commission administrative du musée Thomas Dobrée jusqu'à sa mort, le 11 février 1924, à son domicile nantais du 20 avenue de l'Éperonnière. Contrairement à Fortuné Parenteau, dont le décès passe quasiment inaperçu dans la communauté scientifique, les éloges après la mort de Pitre de Lisle sont nombreux ${ }^{318}$.

315. Dans sa correspondance avec le comte Raoul de Rochebrune qui prend des nouvelles du conservateur, le gardien chef du musée, Rémy Gibouin écrit entre 1901 et 1909 qu'il " se repose à la campagne ". Dans sa "notice nécrologique", Alcide Dortel, président de la Société, nous apprend que "depuis longtemps sa santé déclinait " : BSANLI, 64, 1924, p. 254.

316. BSANLI, 58, 1918, p. LIII, 3 octobre 1918.

317. La dernière séance où sa présence est mentionnée est celle du 5 mai 1909, BSANLI, 50, 1909, p. XIx. Déjà, depuis 1905, il n'assiste plus qu'une ou deux fois par an aux réunions de la Société archéologique.

318. L'Écho de la Loire, "Nécrologie de M. le vicomte P. de Lisle du Dreneuc ", février 1924; Le Phare de la Loire, " Nécrologie, Les obsèques de M. de Lisle du Dréneuc ", avec le discours du préfet Paul Bouju; archives privées, texte prononcé par le président du Conseil 
Le 3 mars 1924, la Commission administrative du musée archéologique et la Société archéologique donnent leur avis sur les deux candidatures à la succession de Pitre de Lisle du Dreneuc, celles du chanoine Georges Durville et du vicomte Paul Aveneau de La Grancière. Son collaborateur, le chanoine Georges Durville, lui succède à la direction des deux musées. Il est nommé conservateur du musée Thomas Dobrée à la séance de la Commission administrative du 6 mars 1924, avec un conservateur adjoint, Paul Aveneau de La Grancière ${ }^{319}$.

De 1856 à 1924, le métier de conservateur et la pratique de l'archéologie ont fondamentalement changé. Si par ses méthodes de travail, la constitution de sa collection et ses publications, Fortuné Parenteau reste un homme du XIX ${ }^{\mathrm{e}}$ siècle, Pitre de Lisle, entraîné par le courant des archéologues de la fin du XIX ${ }^{\mathrm{e}}$ siècle, a innové par une approche scientifique et une méthodologie plus rigoureuse. Même si l'archéologie de terrain les a, un temps, passionnés, ils ont, tous deux, eu pour préoccupation essentielle leur métier de conservateur qu'ils ont pratiqué avec ardeur, lui consacrant une grande partie de leur vie. Pour Fortuné Parenteau, les deux collections, publique et privée, sont liées; pour Pitre de Lisle, la collection privée reste sans doute la part secrète et très chère de sa vie.

La présentation et la publication des collections archéologiques restent au cœur des préoccupations du conservateur, Georges Durville, et de l'administration départementale. L'installation, à partir de 1924, et la publication, en 1927, des collections du musée lapidaire sont très vite suivies, dès 1931, des premiers constats sur l'étroitesse des espaces de présentation de l'archéologie au rez-de-chaussée du palais Dobrée et sur la suggestion d'une construction nouvelle : "Dans les vitrines les objets sont touche à touche; de haut en bas, les murs disparaissent sous les objets qui y sont accrochés, c'est à peine si on peut y trouver place pour quelques clous $^{320}$... " II faudra attendre, vingt ans plus tard, le travail d'inventaire mené par des chargés de mission diligentés par le ministère à la demande du conservateur, Bernard Roy ${ }^{321}$, puis le premier inventaire rétrospectif des collections mené, sous

général, Adolphe Jollan de Clerville, aux obsèques de M. de Lisle du Dreneuc; Alcide Dortel, président de la Société archéologique, " Notice nécrologique ", BSANLI, 64, 1925 (1924), p. 253-254.

319. Archives du musée Dobrée, Commission administrative du musée Thomas Dobrée, 6 mars 1924.

320. Archives du musée Dobrée, Georges Durville, rapport annuel au préfet, 1931, puis 1934, p. 2.

321. Archives du musée Dobrée, Correspondance avec le ministère, années 19451955, courriers avec le conservateur Bernard Roy. Archives du musée Dobrée, CosTA, Dominique, Rapport annuel au préfet, 1955-1956, p. 2-4; des "spécialistes des musées nationaux " sont chargés de l'inventaire et du reclassement des collections : $\mathrm{M}^{\mathrm{me}}$ Cammas (collections d'archéologie régionale, essentiellement préhistoriques), David Weill (collections musulmanes), Paule Krieger (collections égyptiennes), Jacques Lethève (collections des estampes du fonds Dobrée et du musée départemental d'Archéologie), Mme LionGoldschmidt (collections extrême-orientales). 
la direction du nouveau conservateur, Dominique Costa, et son rapport de 1967 sur l'extension des musées départementaux de Loire-Atlantique ${ }^{322}$, pour aboutir à la décision d'une nouvelle construction... prévue, non pas pour les collections archéologiques, mais pour les collections d'ethnographie précolombienne en déshérence dans les musées de province ${ }^{323}$. Ce bâtiment est construit en 1972-1973, mais il est principalement consacré à la présentation de l'archéologie régionale ${ }^{324}$. Malheureusement, la surface consacrée aux collections archéologiques étant nettement insuffisante dès l'origine, l'administration départementale commence à réfléchir moins de dix ans après à un nouveau projet. Confiée à l'architecte Dominique Perrault, la rénovation extension du musée Dobrée devrait s'achever en 2015 placer, à nouveau, les collections archéologiques régionales au cœur de sa nouvelle présentation permanente, et donner à une population redevenue nomade, des clefs de compréhension du territoire, des origines au rattachement de la Bretagne à la France ( $\mathrm{XVI}^{\mathrm{e}}$ siècle).

\section{Bibliographie des travaux de Fortuné Parenteau en archéologie}

Catalogue et description des objets du musée archéologique de Nantes et de la Loire-Inférieure, Nantes, 1856, imprimerie Armand Guéraud, 108 p.

"Fouilles de Pouzauges (Vendée). Attributions gauloises ", BSANLI, 1, 1859-1861, p. 191-208, 4 pl. h.t.

"Fouilles archéologiques (Rezé) ", BSANLI, 1, 1859-1861, p. 459-467, pl. I-II [ce texte est daté de décembre 1861].

"Essai sur les monnaies des Namnètes ", BSANLI, 2, 1862, p. 103-124, pl. I-III [ce texte est daté de février 1863. Par ailleurs, un tirage à part, à cent exemplaires, paginé de 3 à 24, porte la date d'édition de 1863].

"Introduction à l'étude des bijoux ", BSANLI, 3, 1863, p. 73-75.

" Notice sur un atelier de fondeur gallo-romain du I Ir siècle, découvert à Rezé ", $C A F, 31,1864$, Fontenay-le-Comte-Évreux, Falaise, Troyes, 31, 1865, p. 253257.

Essai sur les poteries de l'Ouest de la France, Nantes, Henri Charpentier éditeur, 1865,22 p., pl. I-V.

" Découverte du jardin des Plantes de Nantes. Attributions celtiques ", BSANLI, 8, 1868, p. 19-46, pl. I-IV hors texte. [Un tiré à part a été édité : Le fondeur du Jardin-des-Plantes de Nantes et son confrère de Rezé. Attributions celtiques et gallo-romaines, Nantes, Vincent Forest et Émile Grimaud imprimeurs-éditeurs, 1868, 32 p. et 4, pl.].

322. Archives du musée Dobrée, s.v. Histoire du musée.

323. Presse-Océan, 12 octobre 1971, "Le Musée Dobrée s'agrandit pour accueillir les antiquités précolombiennes réparties à travers la France".

324. Costa, Dominique, "Le nouveau Musée de Nantes ", Revue du Louvre, 1975, 3, p. 211 212. 
Catalogue du musée départemental d'Archéologie de Nantes et de la LoireInférieure. Nantes, imprimerie Vincent Forest et Émile Grimaud, 1869, 140 p., pl. I-XII et 23 dessins.

" Objets divers de l'âge du bronze de Nantes et environs ", Matériaux pour l'histoire primitive et naturelle de l'Homme, 1869, p. 190-192, 1 pl.

"Inscription et tombeaux chrétiens ", BSANLI, 10, 1870-1871, p. 15-19, 3 pl.

"Segora (statio) ", BSANLI, 10, 1870-1871, p. 81-91, pl. I-III.

« Un canon de bronze du siège d'Orléans en 1428 », BSANLI, 10, 1870-1871, p. 133$142,1 \mathrm{pl}$.

Catalogue raisonné de l'exposition des Beaux-Arts, Archéologie et Peinture ancienne, Nantes, imprimerie Jules Grinsard, 1872, 184 p., pl. I-XIII, tiré à 100 exemplaires. [Il rédige une grande partie du catalogue et, notamment, la partie consacrée à l'archéologie, p. 17-162].

"Odyssée de la bécasse en Gaule », BSANLI, 12, 1873, p. 25-28, 1 pl.

"Les cabournes, les bourniers et les bournigals ", BSANLI, 12, 1873, p. 29-30.

" Boîte à feu du XIv e siècle ", BSANLI, 13, 1874, p. 219-220, 1 pl.

" Fouilles du lac de Grand-Lieu. Les lacs de Paladru, et de Grand-Lieu », BSANLI, 14, 1875, p. 147-150 [tiré à part, 1875, 4 p., s.d.].

Inventaire archéologique précédé d'une introduction à l'étude des bijoux. Nantes : Vincent Forest et Émile Grimaud, 1878, 140 p., pl. I-61, tiré à 175 exemplaires. "Les bijoux bretons ", Bretagne artistique, pittoresque et littéraire, 1880, p. 72-80. " Le culte du veau d'or dans l'Antiquité et jusqu'à nos jours (analyse) ", BSANLI, 19, 1880, p. 183-186.

\section{Bibliographie des travaux de Pitre de Lisle du Dreneuc en archéologie}

"Stations paléolithiques et néolithiques de la Loire-Inférieure ", BSANLI, 17, 1878, p. 45-56, 1 pl. [tiré à part, Nantes, Vincent Forest et Émile Grimaud, imprimeurs-éditeurs, 1878, 14 p., 1 pl.].

"Inventaire archéologique par F. Parenteau », BSANLI, 18, 1879, p. 248-250.

" Notes sur Caranda et les poteries mérovingiennes offertes par M. F. Moreau père au musée de Nantes ", BSANLI, 19,1880, p. 107-112.

« Dictionnaire archéologique de la Loire-Inférieure (Époques primitive, celtique, gauloise et gallo-romaine). Arrondissement de Châteaubriant " ( ${ }^{\text {re }}$ partie), BSANLI, 19, 1880, p. 117-182, 2 pl. et tableau des monuments.

"Caractères particuliers des antiquités primitives de la Bretagne ", $B A A B, 1880$, I, p. 113-118 et 2 pl. h.t.

"Une arme historique en pierre polie ", BSANLI, 20, 1881, p. 69-73.

"Fouilles du tumulus de La Roche. Donges, Loire-Inférieure. Notes d'une excursion de G. et P. de Lisle dans l'ancien archipel de la Basse-Loire, Donges, Besné, Crossac, etc. ", BSANLI, 20, 1881, p. 75-88, 2 pl. h.t. [Tiré à part, Nantes, Vincent Forest et Émile Grimaud, imprimeurs-éditeurs, 1882, 14 p. et 2 pl.]. 
" Notes sur différentes armes de pierre et de bronze, trouvées aux environs de Donges (Loire-Inférieure) ", BSANLI, 20, 1881, p. 89-90.

"Les haches à tête de la Bretagne et du Bocage ", Matériaux pour l'histoire primitive et naturelle de l'Homme, XI, 1880, p. 462-486, 3 pl. et tableaux de répartition par département.

Les haches à tête de la Bretagne et de la Vendée. Notes complémentaires, Nantes, Vincent Forest et Émile Grimaud, imprimeurs-éditeurs, mars 1881, non paginé, 4 p. et $1 \mathrm{pl}$.

"La Bretagne primitive. $1^{\mathrm{er}}$ mémoire. La Bretagne avant et pendant la période dolménique ", $B A A B, 1881$, I, p. 51-65, 1 pl.; " $2^{\mathrm{e}}$ mémoire. Les Celtes et les monuments mégalithiques de la Bretagne et de l'Angleterre ", $B A A B$, 1881 , I, p. $66-79$; « $3^{\mathrm{e}}$ mémoire. Projet de restauration du menhir géant de Locmariaker ", $B A A B, 1881$, I, p. 80-84.

"Stations primitives de la Bretagne ", $B A A B, 1882$, II, p. 3-27 et 1 pl. [Tiré à part, Saint-Brieuc, L. Prudhomme, imprimeur, 1883, 25 p. et 4, pl.].

" Découvertes de haches en plomb (Bretagne) ", $R A, 42$, déc. 1881, p. 335-343, 1 fig.

"Les armes de bronze du sud-est et du nord-ouest de la Bretagne ", $B A A B, 1882$, II, p. 28-36, 2 pl., 1 fig., et 1 tableau. [Tiré à part, Saint-Brieuc, L. Prudhomme, imprimeur, 1883, 18 p. et 4 pl.].

" Dictionnaire archéologique de la Loire-Inférieure. Arrondissement de SaintNazaire. $1^{\text {re }}$ partie ", BSANLI, 20, 1882, p. 93-176.

Dictionnaire archéologique de la Loire-Inférieure (Époques celtique, gauloise et gallo-romaine). Arrondissement de Châteaubriant, p. 1-66, avec un tableau; Arrondissement de Saint-Nazaire, p. 69-232, 1 pl.; Arrondissement de Paimboeuf, p. 233-313, 2 pl., Nantes, imprimerie de Vincent Forest et Émile Grimaud, 1882 (Extrait du BSANLI).

"Épées et poignards de bronze du Morbihan et de la Loire-Inférieure ", BSECDN, 1883, XXI, p. 125-150 avec 3 tableaux. [Tiré à part, 26 p.].

" Dictionnaire archéologique de la Loire-Inférieure (Époques celtique, gauloise et gallo-romaine). Arrondissement de Saint-Nazaire. $2^{\mathrm{e}}$ partie ", BSANLI, 21, 1883 , p. 38-118.

" La collections Ch. Seidler au Musée archéologique de Nantes ", BSANLI, 23, 1884, p. 68-70.

"Euvres de M. Paul du Chatellier ", BSANLI, 23, 1884, p. 187-190.

" Dictionnaire archéologique de la Loire-Inférieure (Époques celtique, gauloise et gallo-romaine). Arrondissement de Paimboeuf ", BSANLI, 24,1885, p. 1-81.

" Bretagne. Les dolmens de Saint-Michel-Chef-Chef ", BSANLI, 25, 1886, p. 127134 et 2 pl.

"Fouilles des dolmens du Grand-Carreau-Vert, Saint-Michel-Chef-Chef (LoireInférieure) ", Matériaux pour l'histoire primitive et naturelle de l'Homme, 3, 1886 , p. $277-285$ et 8 fig.

"Les triangles de menhirs de la Loire-Inférieure et les menhirs triangulaires du pays de Retz ", Revue archéologique du Comité des travaux historiques et scientifiques, 1886, 01, p. 139-144 et 1 fig. 
" Des Gaulois Vénètes de la Grande Brière et du théâtre de la bataille navale de Brutus dans les Cornouailles ", $B A A B, 1886$, p. 27-36.

Nouvelles découvertes d'idoles de l'Amazone, Paris, Émile Lechevalier, 1889, 20 p., 2 pl. et 4 fig.

" Nantes à l'époque gallo-romaine par M. Legendre inspecteur diocésain ; compte-rendu ", BSANLI, 29, 1890, p. 146-150.

"Les fouilles du grand monument de Boga en Guérande (Loire-Inférieure) ", BSANLI, 29, 1890, p. 162-165.

" Notes sur le navire sculpté de la rue St-Clément ", BSANLI, 30, 1891, p. 128-132.

" Recherches archéologiques sur les origines de Nantes. Corbilon. $1^{\text {re }}$ partie ", BSANLI, 31, 1892, p. 183-198. [Tiré à part, Vannes, imprimerie Lafolye, 1892, 15 p.].

" Notice sur les fouilles du tumulus de La Motte, Sainte-Marie (Loire-Inférieure) ", $B S A N L I, 31,1892$, p. 199-203, 1 pl. h.t. [Tiré à part, Vannes, imprimerie Lafolye, 1892, 5 p. et 1 pl.].

"Amulette gauloise. Note sur une dent d'ours trouvée dans la Grande-Brière ", BSANLI, 32, 1892, p. 220-222.

Notice sur le musée Th. Dobrée, Nantes, Émile Grimaud éditeur, 1897, 40 p.

"Le musée Thomas Dobrée " et "Le Musée départemental d'Archéologie ", La Ville de Nantes et la Loire-Inférieure, Nantes, imprimerie Émile Grimaud, 1898, II, p. 45-69 et 71-81.

Catalogue du Musée archéologique de Nantes, Nantes, imprimerie Moderne, Joubin et Beuchet Frères, 1903, 376 p.

Catalogue des collections archéologiques de Mrs Georges et Pitre de Lisle du Dreneuc, Nantes, imprimerie Moderne, 1903, 19 p., 6 pl.

Catalogue général des collections du musée Th. Dobrée, Nantes, imprimerie Moderne, Joubin et Beuchet Frères, 1906, 1019 p.

"Épée avec inscription en relief ", BACTH, 1912, p. 130-131.

« Note sur deux épées gauloises trouvées dans la Loire ", BACTH, 1913, p. 14-16 et $1 \mathrm{pl}$. [Tiré à part, Paris, Imprimerie nationale, 1913, 3 p. et $1 \mathrm{pl}$.].

"Épée gauloise trouvée au Pont de l'Ouen (Loire-Inférieure), $3^{\mathrm{e}}$ période du Hallstatt, $\mathrm{VII}^{\mathrm{e}}-\mathrm{VII}^{\mathrm{e}}$ siècle avant J.-C. ", L'Homme préhistorique, 6, 1914, p. 161166. [Tiré à part, Paris, Librairie J. Gamber, 1914, 6 p. et 1 pl.]. 


\section{RÉSUMÉ}

Parmi les pionniers de l'archéologie nantaise et départementale de la seconde moitié du $\mathrm{XIX}^{\mathrm{e}}$ siècle et du début du $\mathrm{XX}^{\mathrm{e}}$ siècle, deux personnalités ont profondément marqué la création des collections du musée départemental d'Archéologie de la Loire-Inférieure, aujourd'hui le musée Dobrée. Fortuné Parenteau et Pitre de Lisle du Dreneuc sont issus d'un milieu aisé et fréquentent les sociétés savantes où il est de bon ton de pratiquer l'archéologie et de constituer des collections. Leur formation intellectuelle leur permet de les cataloguer, de les documenter et de les dessiner, et leurs archives constituent une source fondamentale pour l'histoire de l'archéologie locale et des collections, même si, parfois, il est bien difficile de savoir où finissent leurs propres collections et où commencent celles du musée.

\section{ABSTRACT}

Among the pioneers of archeology in Nantes and its département during the late 19th century and early $20^{\text {th }}$ Century, two important people strongly influenced the creation of the Musée départemental d'Archéologie de la Loire-Inférieure, which is today the Musée Dobrée. Fortuné Parenteau and Pitre de Lisle du Dreneuc both were of a well-to-do background and associated with learned societies in which it was fashionable to practice archeology and build up collections. Their intellectual background enabled them to catalogue, document, and draw their collections. Furthermore, their archives constitute a fundamental source for the history of local archaeology and collections, even if, sometimes, it is difficult to distinguish where their private collections end and where the museum collections begin. 